\title{
DUALITY FOR REGULAR SYSTEMS OF WEIGHTS*
}

\author{
KYOJI $\mathrm{SAITO}^{\dagger}$
}

\begin{abstract}
Regular systems of weights are certain combinatorial and arithmetic objects related to a generalization of Coxeter elements [S6,7,8 and 11], and introduced in motivation to understand the flat structure for primitive forms for isolated hypersurface singularities [S3] (cf. [Man],[S11]).

In the present article, the theory is applied to explain the self-duality of ADE (=simply laced Dynkin diagrams) and the strange duality of Arnold. Beyond the original applications, the study gives further class of dual weight systems, which, for instance, has close connection with Conway group and seems interesting to be studied yet further.
\end{abstract}

On the other hand, the duality of weight systems has an interpretation in terms of certain products of Dedekind eta functions. We give a conjecture on the non-negativity of the Fourier coefficients of the eta-products. The conjecture is solved affirmatively for the cases corresponding to elliptic root systems [S45]. But the meaning is not yet clear.

Recently, one finds an equivalence between the duality in the present article and certain string duality in mathematical physics [T].

0. Introduction. The present article gives a general frame work on the duality of regular systems of weights. For a sake of self-containedness, all proofs are given or sketched except for some basic facts. For simplicity, we shall call a regular system of weights a weight system unless otherwise is stated.

A weight system $W:=(a, b, c ; h)$ is a system of 4 positive integers with some arithmetic constraint (see (1.0)). To $W$, we attach a cyclotomic polynomial $\varphi_{W}$, called the characteristic polynomial of the weight system (see (2.1)). The duality we study in the present article appears as a duality between the cyclotomic polynomials. Let us explain this by examples.

Let $h$ be a positive integer and let $\varphi(\lambda)$ and $\varphi^{*}(\lambda)$ be cyclotomic polynomials whose roots are $h$-th roots of unity. The polynomials can be decomposed in the form:

$$
\varphi(\lambda)=\prod_{i \mid h}\left(\lambda^{i}-1\right)^{e(i)} \quad \text { and } \quad \varphi^{*}(\lambda)=\prod_{i \mid h}\left(\lambda^{i}-1\right)^{e^{*}(i)},
$$

where $e(i), e^{*}(i) \in \mathbb{Z}$ for $i \in \mathbb{N}$ with $i \mid h$. We call $\varphi$ and $\varphi^{*}$ to be dual to each other of level $h$, if the equality

$$
e(i)+e^{*}(h / i)=0
$$

holds for all $i$. We give two examples of such dualities between cyclotomic polynomials, which motivated the present article.

1. The characteristic polynomial of the Coxeter element in the Weyl group of a type $A_{l}(l \geq 1), D_{l}(l \geq 4)$ or $E_{l}(l=6,7,8)$ is self-dual (see Remark 0.11 and $\left.\S 11\right)$.

${ }^{*}$ Received November 2, 1998; accepted for publication January 5, 1999. The present article is an extended version of [S10]. A precis has appeared in [S12].

†Research Institute for Mathematical Sciences, Kyoto University, Kitashirakawa, Sakyo-ku, Kyoto, 606-01, Japan (saito@kurims.kyoto-u.ac.jp). 
2. The characteristic polynomials of the Milnor monodromies ([M, §9]) for the strange dual pair of 14 exceptional unimodular singularity ([A1]) are dual to each other (see $\S 12)$.

Both facts 1 . and 2. can be easily verified by direct calculations. On the other hand, the characteristic polynomials in the above 1 . and 2 . can be realized as these attached to some special weight systems (see $\S 9,12$ ). Then the duality 1 . and 2 . are proven uniformly without using the classification as a consequence of a general frame work of the duality of weight systems ( $\$ 7$ Theorem 7.10 ) developed in the present article $\S \S 3-7$.

Beyond the above examples, the general framework gives further dual pairs of weight systems ( $\$ 7$ Theorem 7.9). In particular, it is remarkable that all weight systems of rank 24 are self-dual and have relationship with Frame shapes of Conway group (cf. Appendix 1), whose study in connection with mirror symmetry seems highly interesting (cf. Appendix 2).

The duality between weight systems is reformulated $(\S 7)$ as the duality of the set $M(\varphi):=\left\{i \in \mathbb{Z}_{>0} \mid e(i) \neq 0\right\}$, since the set $M(W)$ attached to the characteristic polynomial for a weight system $W$ admits a priori description from the weights $(\S 4)$ and carries a specific structure such as a poset with 3 generators, classified into 14 types, and the sign of $e_{W}(i)$ is determined by the level function $n$ on the poset $M(W)$ (Theorem 5.1-2).

The $\S \S 8-10$ are devoted for a study of some numerical invariants attached to a weight system: dual rank $\nu_{W}$, genus $a_{0}$, discriminants $d(W)$ and $d^{*}(W)$ and signature $A(W)$, which are used in $\S$ 's 12 and 13.

Attached to the decompositions of $\varphi(\lambda)$ and $\varphi^{*}(\lambda)$, define the products:

$$
\eta_{\varphi}(\tau):=\prod_{i \mid h} \eta(i \tau)^{e(i)} \quad \text { and } \quad \eta_{\varphi *}(\tau):=\prod_{i \mid h} \eta(i \tau)^{e^{*}(i)}
$$

where $\eta(\tau):=q^{1 / 24} \prod_{n=1}^{\infty}\left(1-q^{n}\right)$ for $q=\exp (2 \pi \sqrt{-1} \tau)$ is the Dedekind eta-function. Then the duality between $\varphi$ and $\varphi^{*}$ is equivalent to:

$$
\eta_{\varphi}(-1 / h \tau) \cdot \eta_{\varphi *}(\tau) \cdot \sqrt{d}=1
$$

where $d$ is the discriminant. It is also elementary to see that the Fourier coefficients at $\infty$ of such dual pair of the eta-products are non-negative. Inspired by these facts, the $\S 13$ is devoted for the study of the eta-product $\eta_{W}$ attached to $\varphi_{W}$ of a weight system $W$. We show that $\eta_{W}$ is holomorphic (resp. vanishing) at all cusps if and only if one has an inequality $\nu_{W} \leq 0$ (resp. $\nu_{W}<0$ ). We ask:

Conjecture. The eta-product $\eta_{W}$ has non-negative Fourier coefficients at $\infty$ if and only if $\nu_{W}$ is non-negative (i.e. $\eta_{W}$ is non-cuspidal).

For three elliptic weight systems $\widetilde{E}_{6}, \widetilde{E}_{7}, \widetilde{E}_{8}$ and for a weight system $(1,1,2 ; 5)$ (whose eta-product is the one studied by Ramanujan), the conjecture is verified by a use of Euler products for the associated Dirichelet series (Example 14.3) (see [S41-V] for a complete treatment of the elliptic case).

REMARK 0.1 .

1. The concept of a regular system of weights is an axiomatization of weight systems of a polynomial of 3 variables defining a weighted homogeneous isolated surface singularity (see [S6,7,11], Remarks 1.3, 2.1,10.3, §12 and Appendix 2). The 
present paper is constructed axiomatically without assuming any knowledge of the back ground except for $\S 12$.

2. The duality for Coxeter elements explained here is equivalent to a duality on Cartan matrix observed by Ochiai [Oc] (see §11).

Acknowledement. The interpretation of the duality in terms of eta-products was suggested by G. Mason ([Ma]). The author is grateful to him for the discussions.

1. Regular system of weights. We recall basic definitions and results from [S6,7 and 8].

A system of 4 integers $a, b, c$ and $h$ satisfying $0<a, b, c<h$ :

$$
W:=(a, b, c ; h)
$$

is called $a$ weight system, where $a, b$ and $c$ are called the weights and $h_{W}:=h$ is called the Coxeter number. Two weight systems are identified when they have the same set of weights and the same Coxeter number.

For a weight system $W:=(a, b, c ; h)$, we associate a rational function:

$$
\chi_{W}(T):=T^{-h} \frac{\left(T^{h}-T^{a}\right)\left(T^{h}-T^{b}\right)\left(T^{h}-T^{c}\right)}{\left(T^{a}-1\right)\left(T^{b}-1\right)\left(T^{c}-1\right)}
$$

in an indeterminate $T$. We say that $W$ is regular, if $\chi_{W}(T)$ has a pole at most at $T=0$, or equivalently, if $T^{h} \chi_{W}(T)$ is a polynomial in $T$ [S6 (1.2)].

The regularity condition is reformulated in terms of elementary arithmetics: a weight system $(a, b, c ; h)$ is regular, if and only if i) each of $a, b$, and $c$ divides one of $h-a, h-b$ or $h-c$, and ii) every $\operatorname{gcd}(a, b), \operatorname{gcd}(b, c)$ and $\operatorname{gcd}(c, a)$ divides $h$. So, unless all weights are less than or equal to $h / 2$, there are two weights whose sum is equal to $h$. The weight system of the latter case (e.g. $b+c=h$ ) will be called of type $A_{\ell}$ where $\ell:=h / a-1$ for a reason explained below. As said in the introduction, from now on we shall call a regular system of weights $W$ simply a weight system $W$.

For a weight system $W$, it was shown [S6 (1.3) Theorem] that there is a finite number of integers $m_{1}, \cdots, m_{\mu}$ such that

$$
\chi_{W}(T)=T^{m_{1}}+\cdots+T^{m_{\mu}},
$$

where

$$
\mu=\mu_{W}=(h-a)(h-b)(h-c) / a b c
$$

is called the rank of the weight system. The integers $m_{1}, \cdots, m_{\mu}$ are called the exponents for the weight system $W$ (see Remark 1.3). Otherwise stated, we order them as $m_{1} \leq m_{2} \leq \cdots \leq m_{\mu}$. Since one has $T^{h} \chi_{W}\left(T^{-1}\right)=\chi_{W}(T)$, one obtains: $a n$ additive duality of the exponents:

$$
m_{i}+m_{\mu-i+1}=h \quad \text { for } i=1, \cdots, \mu .
$$

The Laurent expansion of (1.1) shows that the smallest exponent $m_{1}$, which we denote by $\varepsilon_{W}$, is given by

$$
\varepsilon_{W}:=a+b+c-h,
$$

and that its multiplicity is 1 (i.e. $m_{1}<m_{2}$ and $m_{\mu-1}<m_{\mu}$ if $\mu \geq 2$ ). 
For a positive integer $d$, the weight system $(d a, d b, d c ; d h)$ is regular if and only if $(a, b, c ; h)$ is regular. So, we shall call a weight system is primitive, if either when $W$ is not of type $A_{\ell}$ and $\operatorname{gcd}(a, b, c, h)=1$, or $W$ is of type $A_{\ell}$ of the form $(1, b, \ell+1-b ; \ell+1)$ for some $b$ with $1 \leq b \leq \ell$. In fact, one has $(a, b, c)=1$ in both cases.

Primitive weight systems having only positive exponents are classified, and are called of types $A_{\ell}(\ell \geq 1), D_{\ell}(\ell \geq 4)$ and $E_{\ell}(\ell=6,7,8)$ according to the identification of the set of exponents with that of corresponding root systems (see [S6, §2] and §11). It is well known that the smallest exponent for these cases is equal to 1 ([Bo, Ch.V, $\S 6$ 6.2. Theoreme 1. (i)]). In general, for a primitive weight system, it is an important question whether the exponent equal to 1 or -1 exists (see Remark 2.1). In [S8 (2.2)], we answered to the question affirmatively as follows.

TheOREM 1.1. Let $W:=(a, b, c ; h)$ be a primitive weight system. Put

$$
a_{m}:=\text { the multiplicity of the exponent equal to } m \text {. }
$$

Then either $a_{1}>0$ or $a_{-1}>0$. That is:

$$
\operatorname{mult}(W):=a_{1}+a_{-1}>0 \text {. }
$$

Here we call mult $(W)$ the multiplicity of $W$. Later in $\S 5$, the multiplicity is identified with an exponent $e_{W}(h)$ (5.6). Then the above theorem will be reproved as the positivity of the exponent $e_{W}(h)$ (see Theorem 5.1 and Remark 5.41 , cf. Remark 2.1).

Later in $\S 6$, a weight system $W$ is called simple, if mult $(W)=1$. The following imediate consequence of Theorem 1.1 is used in $\S 6$.

ASSERTION 1.2. Let $W$ be a primitive weight system, whose exponents are positive. Then $W$ is simple and the smallest exponent $\varepsilon_{W}$ is equal to 1.

Proof. Since $a_{-1}=0$, mult $(W)=a_{1}>0$ implies the existence of exponent equal to 1 . Of course 1 should be the smallest exponent. Then the simplicity of the multiplicity of the smallest exponent (1.5) implies $a_{1}=1$ and hence the simplicity of $W$.

REMARK 1.3. Let $f_{W}(x, y, z)=\sum_{a i+b j+c k=h} c_{i j k} x^{i} y^{j} z^{k}$ be a weighted homogeneous polynomial in three variables $(x, y, z)$ of weights $(a, b, c)$ with the total degree $h$. Then, for a generic choice of the coefficients, the surface $X_{W, 0}$ defined by the equation $f_{W}(x, y, z)=0$ has an isolated singularity at the origin if and only if the weight system $(a, b, c ; h)$ is regular (c.f. [A3], [S6, Theo. 3]). If one assume this fact, then (1.2) and (1.3) are trivialities, since $\chi_{W}(T)$ becomes a Poincaré polynomial for the finite dimensional Jacobian ring $\mathbf{C}[x, y, z] /\left(\partial_{x} f_{W}, \partial_{y} f_{W}, \partial_{z} f_{W}\right)$ (up to the factor $\left.T^{\varepsilon_{W}}\right)$.

2. Characteristic polynomial $\varphi_{W}(\lambda)$. Let the notation be as in $\S 1$. The characteristic polynomial for a weight system $W$ is defined by

$$
\varphi_{W}(\lambda):=\prod_{i=1}^{\mu}\left(\lambda-\omega_{i}\right)
$$

where

$$
\omega_{i}:=\exp \left(2 \pi \sqrt{-1} m_{i} / h\right) \quad i=1, \cdots, \mu,
$$

and $m_{i}$ are the exponents introduced in $\S 1$ (1.2). Let us prove $\varphi_{W} \in \mathbb{Z}[\lambda]$ in the following (2.3)-(2.5). For $k \in \mathbb{Z}$ consider the sum:

$$
A_{W}(k):=\omega_{1}^{k}+\cdots+\omega_{\mu}^{k}
$$


In view of $(1.2)$, one has $A_{W}(k)=\chi_{W}\left(\omega^{k}\right)$ for $\omega:=\exp (2 \pi \sqrt{-1} / h)$. On the other hand, the substitution of $T=\omega^{k}$ in (1.1) yields

$$
\chi_{W}\left(\omega^{k}\right)=\left(\delta(k a \bmod h) \frac{h}{a}-1\right)\left(\delta(k b \bmod h) \frac{h}{b}-1\right)\left(\delta(k c \bmod h) \frac{h}{c}-1\right)
$$

where $\delta(x):=1$ or 0 according as $x=0$ or else.

Proof of (2.4). Let us show that the function $\psi(T):=\left(T^{h}-T^{a}\right) /\left(T^{a}-1\right)$ takes value $\delta(k a \bmod h) \frac{h}{a}-1$ at $T=\omega^{k}$. Since $T^{h}$ takes value 1 at $T=\omega^{k}$, the values of the denominator and the numerator of $\psi(T)$ at $T=\omega^{k}$ are the same but with opposite sign. If both are not zero, then $\psi\left(\omega^{k}\right)=-1$. They are zero when $\omega^{k a}=1$, i.e. when $\delta(k a \bmod h)=1$. Then the value of $\psi$ is the ratio of the derivatives: $\left(T^{h}-T^{a}\right)^{\prime} /\left(T^{a}-1\right)^{\prime}=\left(h T^{h-1}-a T^{a-1}\right) / a T^{a-1}=h / a-1$. Performing similar calculations for other factors of $\chi_{W}$, we complete the proof. $\square$

Comparing the fact $A_{W}(k)=\chi_{W}\left(w^{k}\right)$ and (2.4), we obtain

$$
A_{W}(k)=\left(\delta(k a \bmod h) \frac{h}{a}-1\right)\left(\delta(k b \bmod h) \frac{h}{b}-1\right)\left(\delta(k c \bmod h) \frac{h}{c}-1\right) .
$$

Hence $A_{W}(k)$ are rational numbers. So, one has $\varphi_{W} \in \mathbb{Q}[\lambda]$. By definition, $\varphi_{W}$ is a monic polynomial and hence is a product of irreducible cyclotomic polynomials (which are monic and integral). The (2.5) implies:

$$
A_{W}(1):=\omega_{1}+\cdots+\omega_{\mu}=-1 .
$$

REMARK 2.1. Under the notation of Remark 1.3, the rank $\mu_{W}$ is equal to the rank of the middle homology group, or of the lattice of vanishing cycles in a smoothing of $X_{W, 0}$, and is called the Milnor number. The characteristic polynomial of Milnor's monodromy $c_{W}$ acting on the lattice (which is semi-simple of order $h$ ) coincides with $\varphi_{W}(\lambda)([\mathrm{M}])$. The existence of exponents 1 or -1 , shown in (1.7), implies that the monodromy $c_{W}$ has the $h$-th primitive root of unity $\exp ( \pm 2 \pi \sqrt{-1} / h)$ as an eigenvalue.

Conjecture. The eigenspace belonging to the primitive roots of unity is regular in the sense that the eigenspace should not be contained in the reflexion hyperplane w.r.t. any vanishing cycle if $a_{0}=0$.

The conjecture is affirmatively answered for the positive definite cases $A D E$ in [Bo,Ch.V, $\S 6]$ (cf. [S9]), for the positive semidefinite case $\widetilde{E}_{l}$ for $l=6,7,8$ in $[\mathrm{S} 41, \S 10]$ (a modification of the conjecture is necessary if $a_{0} \neq 0$ ) and for the 14 exceptional cases (unpublished note). The conjecture has quite important consequence in the study of period map associated to a primitive form [S3]. Namely, it implies the existence of flat structure on the invariant ring of the monodromy group. (cf. [S41-S45,S9]).

3. Cyclotomic exponents $e_{W}(\xi)$. Any monic cyclotomic polynomial $\varphi(\lambda)$ has a unique expression:

$$
\varphi(\lambda)=\prod_{i \mid h}\left(\lambda^{i}-1\right)^{e(i)}
$$

for some integers $e(i) \in \mathbb{Z}$ for $i \in \mathbb{N}$ with $i \mid h$. (Here $h$ is an integer such that all roots of $\varphi(\lambda)=0$ are $h$-th roots of unity.) The exponents $e(i)$ are given by the following formulae:

$$
m e(m)=\sum_{d \mid m} \mu(d) A(m / d)
$$




$$
A(k)=\sum_{d \mid k} d e(d)
$$

for $m, k \in \mathbb{N}$. Here the summation index $d$ runs over positive integers, $A(k)$ is the sum of $k$-th powers of roots of $\varphi(\lambda)=0$ (cf. (2.3)) and $\mu(d)$ denotes the Möbius function in $d \in \mathbb{Z}$ (cf. [H, ch.II]).

Proof. Both side of (3.2) are additive with respect to the product of cyclotomic polynomials. Hence it is sufficient to verify the formula only for $\varphi(\lambda)=\lambda^{i}-1$, $i \in \mathbb{N}$. The LHS is $i \delta_{m, i}(\delta=$ Kronecker's delta). Since $A(m / d)$ is $i$ or 0 according as $i \mid(m / d)$ or not, the RHS is either 0 in case $i \nmid m$, or equal to $i \cdot \sum \mu(d)$ where $d$ runs in $\left\{d \in \mathbb{Z}_{\geq 1}: d \mid(m / i)\right\}$ in case $i \mid m$. Due to a property of the Möbius function ([H (2.2)]) this is zero except for the case $m / i=1$. The (3.2)* is obtained from (3.2) by a use of the Möbius inversion formula.

For a weight system $W$, we decompose the characteristic polynomial $\varphi_{W}$ as in (3.1). We shall call the exponent $e(i)$ the cyclotomic exponent for the weight system $W$ and denote it by $e_{W}(i)$. So, combining (3.2) with (2.5), we obtain explicit formulae for the exponents $e_{W}(i)$. Putting $k=0$ in $(3.2)^{*}$, one obtains an expression of the rank:

$$
\mu_{W}=\sum_{i \mid h} i e_{W}(i)
$$

which is nothing but the $\operatorname{deg}\left(\varphi_{W}\right)$. The multiplicity of the root 1 in $\varphi_{W}(\lambda)=0$ is equal to $a_{0}+a_{h}=2 a_{0}$ (recall the fact $-h<m_{1}, \cdots, m_{\mu}<2 h$ ) and is given by

$$
2 a_{0}=\sum_{i \mid h} e_{W}(i) .
$$

The $a_{0}$ is called the genus of the weight system (cf. Remark 10.32).

4. Poset $M(W)$. For a primitive weight system $W$, we attach a poset $M(W)$ consisting of some finite number of positive integers such that the cyclotomic exponent $e_{W}$ can be regarded as a nowhere vanishing function on $M(W)$. From now on, we assume that the weight systems are primitive.

For $h \in \mathbb{Z}_{>0}$, denote by Div $(h)$ the poset consisting of all positive integral divisors of $h$, where the incidence relation $p \prec q$ for $p, q \in \operatorname{Div}(h)$ holds iff $p \mid q$. So $p \vee q:=$ $\operatorname{lcm}(p, q)$ and $p \wedge q:=\operatorname{gcd}(p, q)$. The unit 1 is the minimum and $h$ is the maximum element in $\operatorname{Div}(h)$. For a triplet $\underline{p}=\left(p_{1}, p_{2}, p_{3}\right)$ of integers with $p_{i} \geq 2(i=1,2,3)$ and $\operatorname{lcm}(\underline{p})=h$, put

$$
M(\underline{p}):=\text { the subposet of } \operatorname{Div}(h) \text { generated by } p_{i}(i=1,2,3) .
$$

That is: $M(\underline{p})=\left\{1, p_{i}(1 \leq i \leq 3), p_{i j}:=p_{i} \vee p_{j}(1 \leq i, j \leq 3)\right.$ and $p_{123}:=$ $\left.p_{1} \vee p_{2} \vee p_{3}=\bar{h}\right\}$. So $\sharp M(\underline{p}) \leq 8$. The projection map

$$
\Phi: \operatorname{Div}(h) \rightarrow M(\underline{p}), \quad d \mapsto \max \{\xi \in M(\underline{p}): \xi \mid d\}
$$

is a poset homomorphism (i.e. the incidence relations are preserved).

Let $\widetilde{\mu}(\xi, \eta)$ for $\xi, \eta \in M(p)$ be the Möbius function (cf. [H]). That is: $\widetilde{\mu}$ is an integral valued function on $\overline{M(p)} \times M(\underline{p})$ such that $\widetilde{\mu}(\xi, \eta)=0$ for $\xi \nmid \eta, \widetilde{\mu}(\xi, \xi)=1$ for $\xi \in M(\underline{p})$, and $\sum_{\xi|\lambda| \eta} \widetilde{\mu}(\xi, \bar{\lambda})=\sum_{\xi|\lambda| \eta} \widetilde{\mu}(\lambda, \eta)=0$ for all $\xi$ and $\eta \in M(\underline{p})$ with $\xi_{\neq}^{\mid} \eta$ (here by the notation $\xi_{\neq}^{\mid} \eta$, we mean $\xi \mid \eta$ and $\xi \neq \eta$ ). Let us extend the 
domain of $\tilde{\mu}$ from $M(\underline{p}) \times M(\underline{p})$ to $M(\underline{p}) \times \operatorname{Div}(h)$ by putting $\widetilde{\mu}(\xi, \eta)=0$ for $(\xi, \eta) \epsilon$ $M(\underline{p}) \times(\operatorname{Div}(h) \backslash M(\underline{p}))$.

Assertion 4.1. For any $(\xi, \eta) \in M(\underline{p}) \times \operatorname{Div}(h)$, one has

$$
\sum_{\substack{d \mid \eta \\ d \in \Phi^{-1}(\xi)}} \mu(\eta / d)=\tilde{\mu}(\xi, \eta) .
$$

Proof. Consider a function $f(\xi, \eta):=\sum_{\xi|d| \eta} \mu(\eta / d)$ in $(\xi, \eta) \in M(\underline{p}) \times \operatorname{Div}(h)$. Clearly, $f(\xi, \eta)$ is 0 unless $\xi \mid \eta$ and is 1 if $\xi=\eta$. If $\xi_{\neq}^{\mid} \eta$, then there is a prime number $p$ such that its exponent $e$ in $\eta$ is strictly larger than that in $\xi$. Divide the set of running index $d$ in the definition of $f(\xi, \eta)$ into three parts I; II and III according to the exponent of $p$ in $d$ equals to $e, e-1$ or less than $e-1$. Owing to a property of the Möbius function, the sums over I and II cancel each other and each term of III is zero, so $f(\xi, \eta)=0$. Thus $f(\xi, \eta)=\delta(\xi, \eta)$ (= delta function) for any $(\xi, \eta)$. Decomposing $\left\{d \in \mathbb{Z}_{\geq 0}: \xi|d| \eta\right\}=\underset{\substack{\xi|\lambda| \eta \\ \lambda \in M(\underline{p})}}{\bigcup} \Phi^{-1}(\lambda)$, one has the formula:

$$
\sum_{\substack{\xi|\lambda| \eta \\ \lambda \in M(\underline{p})}} \sum_{\substack{d \mid \underline{\eta}_{1} \\ d \in \Phi^{-1}(\lambda)}} \mu(\eta / d)=\delta(\xi, \eta) .
$$

Consider this as summation for a function in $\lambda \in M(\underline{p})$ for each fixed $\eta$, and apply the inversion formula of Möbius on $M(\underline{p})$. $\mathrm{C}$

Let $W:=(a, b, c ; h)$ be a primitive weight system not of $A_{l}$-type. Put

$$
h / a=p_{1} / q_{1}, \quad h / b=p_{2} / q_{2} \quad \text { and } h / c=p_{3} / q_{3},
$$

where RHS are reduced expressions: that is: $p_{i}, q_{i} \in \mathbb{Z}_{>0}$ with

$$
\left(p_{i}, q_{i}\right)=1
$$

for $i=1,2,3$. So, $p_{1}=h /(h, a), q_{1}=a /(h, a), p_{2}=h /(h, b), q_{2}=b /(h, b)$ and $p_{3}=h /(h, c), q_{3}=c /(h, c)$. By definition, $p_{i} \mid h$ for $i=1,2,3$. One has inequalities and an equality among them:

$$
\begin{gathered}
p_{i} \geq 2 q_{i} \geq 2 \quad(i=1,2,3) \\
\operatorname{lcm}\left(p_{1}, p_{2}, p_{3}\right)=h
\end{gathered}
$$

Proof. (4.6) is trivial from the fact: $h / a, h / b, h / c \geq 2$. Since $p_{i} \mid h(i=1,2,3)$, LHS of (4.7) divides the RHS. Primitivity of $W$ implies $(a, b, c)=1$. This implies that the RHS divides LHS.

The regularity " $(a, b),(b, c)$ and $(c, a)$ divide $h$ " implies the relation

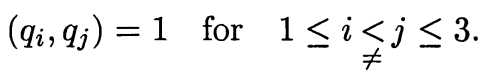

For a primitive weight system $W$, we put

$$
M(W):= \begin{cases}\{1, h\} & \text { if } W \text { is of type } A_{l}, \\ M(\underline{p}) & \text { else. }\end{cases}
$$


Lemma 4.2. For a primitive weight system $W$, let $\tilde{\mu}$ be the Möbius function (cf. [H]) for the poset $M(W)$. Let us extend the domain of $\tilde{\mu}$ from $M(W) \times M(W)$ to $M(W) \times \operatorname{Div}(h)$ by putting $\tilde{\mu}(\xi, \eta)=0$ for $(\xi, \eta) \in M(W) \times(\operatorname{Div}(h) \backslash M(W))$. Then for any $\eta \in \operatorname{Div}(h)$, the cyclotomic exponent $e_{W}(\eta)$ defined in (3.1) is given by

$$
\eta e_{W}(\eta)=\sum_{\xi \in M(W)} A_{W}(\xi) \tilde{\mu}(\xi, \eta)
$$

where $A_{W}(\xi)$ is given in (2.5).

Proof. Rewriting the formula (2.5) in terms of $p_{i}$ and $q_{i}$, one observes that the value $A_{W}(d)$ for $d \in \operatorname{Div}(h)$ depends only on $\Phi(d)$, i.e. $A_{W}(d)=A_{W}(\Phi(d))$. So, formula (3.2) for $e_{W}(\eta)$ can be rewritten as

$$
\begin{aligned}
\eta e_{W}(\eta) & =\sum_{d \mid \eta} \mu(d) A(\eta / d)=\sum_{d \mid \eta} \mu(\eta / d) A(d)=\sum_{d \mid \eta} \mu(\eta / d) A(\Phi(d)) \\
& =\sum_{\xi \in M(W)}\left(A(\xi) \sum_{\substack{d \mid \eta \\
d \in \Phi^{-1}(\xi)}} \mu(\eta / d)\right)=\sum_{\xi \in M(W)}(A(\xi) \widetilde{\mu}(\xi, \eta)) .
\end{aligned}
$$

As a consequence of the lemma, we see that the cyclotomic exponent $e_{W}(\eta)$ is non zero only when $\eta$ belongs to $M(W)$. So, we shall regard the exponent $e_{W}$ as a function on $M(W)$. Nowhere vanishing property of $e_{W}$ as a function on $M(W)$ will be proven in the next section.

5. The sign of the cyclotomic exponent. We introduce a level $n$ (5.1) on the poset $M(W)$, and classify the isomorphism classes of the pair $(M(W), n)$ into 14 types (Theorem 5.2). The cyclotomic exponent $e_{W}$ is calculated as a nowhere vanishing function on $M(W)$ according to the types. We prove the basic result of the present paper: the sign of $e_{W}(\xi)$ at $\xi \in M(W)$ is equal to $(-1)^{n(\xi)+1}$ (Theorem 5.1). In particular, this includes the positivity of $e_{W}(h)$, since $n(h)=3$.

The level of $\xi \in M(W)$ is defined by

$$
\left.n(\xi):=\sharp\left\{i \in\{1,2,3\}: p_{i} \mid \xi\right)\right\},
$$

One has $0 \leq n(\xi) \leq 3$ and $n(\xi)=0$ (resp. 3) if and only if $\xi=1$ (resp. $\xi=h$ ). It is clear that $\xi \mid \eta$ implies $n(\xi) \leq n(\eta)$ and the equality holds if and only if $\xi=\eta$.

THEOREM 5.1. Let $W$ be a primitive weight system.

1. The cyclotomic exponent $e_{W}(\xi)$ is non zero for all $\xi \in M(W)$. The sign of $e_{W}(\xi)$ is equal to $(-1)^{n(\xi)+1}$.

2. One has $e_{W}(\xi)=(-1)^{n(\xi)+1}$ for $\xi \in M(W)$ if $n(\xi)=0$ or 1 .

Theorem 5.1 is a consequence of the next classification Theorem 5.2.

Terminology. An isomorphism class of leveled posets $(M(W), n)$ shall be called a type, where two leveled posets are isomorphic if there is an abstract isomorphism of posets which preserves the level.

THEOREM 5.2. There are 14 types of leveled posets $(M(W), n)$, which are numbered from I to XIV. For each type, the cyclotomic exponent $e_{W}$ is given as a rational function in the coordinates $p_{i}, p_{i j}, h=p_{123}$ and $q_{1}, q_{2}, q_{3}$.

In the next Table $\mathrm{A}$, we exhibit the 14 type posets together with their cyclotomic exponents as the rational function in the coordinate, where 
i) An element $\xi \in M(W)$ is represented by a vertex ( $\xi$ of a graph. The vertices are ordered from left to right according to the level $0 \leq n \leq 3$,

ii) An edge ( $\xi$ for $\xi, \eta \in M(W)$ is drawn, if and only if $\xi \neq \eta$ and there does not exist $\zeta \in M(W)$ such that $\xi \neq \zeta \neq \eta$.

iii) The function attached near at a vertex $\left(\xi\right.$ is the exponent $e_{W}(\xi)$ (in Table $G$, we shall describe the exponent in refined coordinates).

iv) The first 5 types $I-V$ are called dual type for the reason explained in Remark 2 at the end of this $\S$.

Table A.

I.

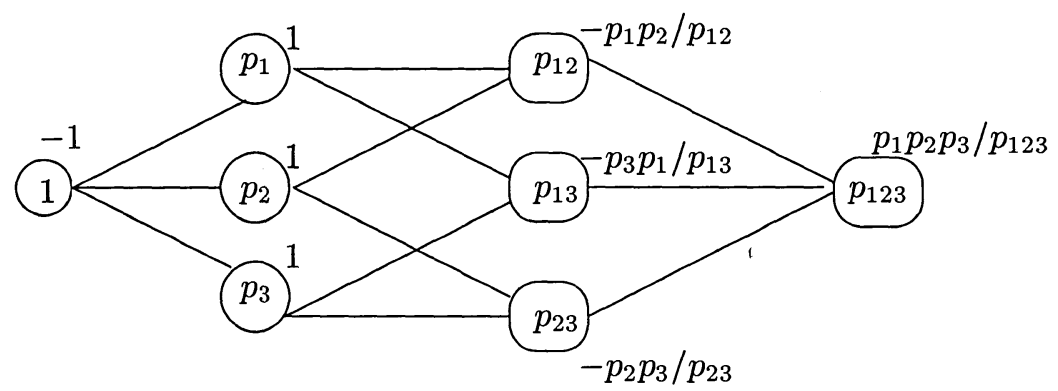

II.

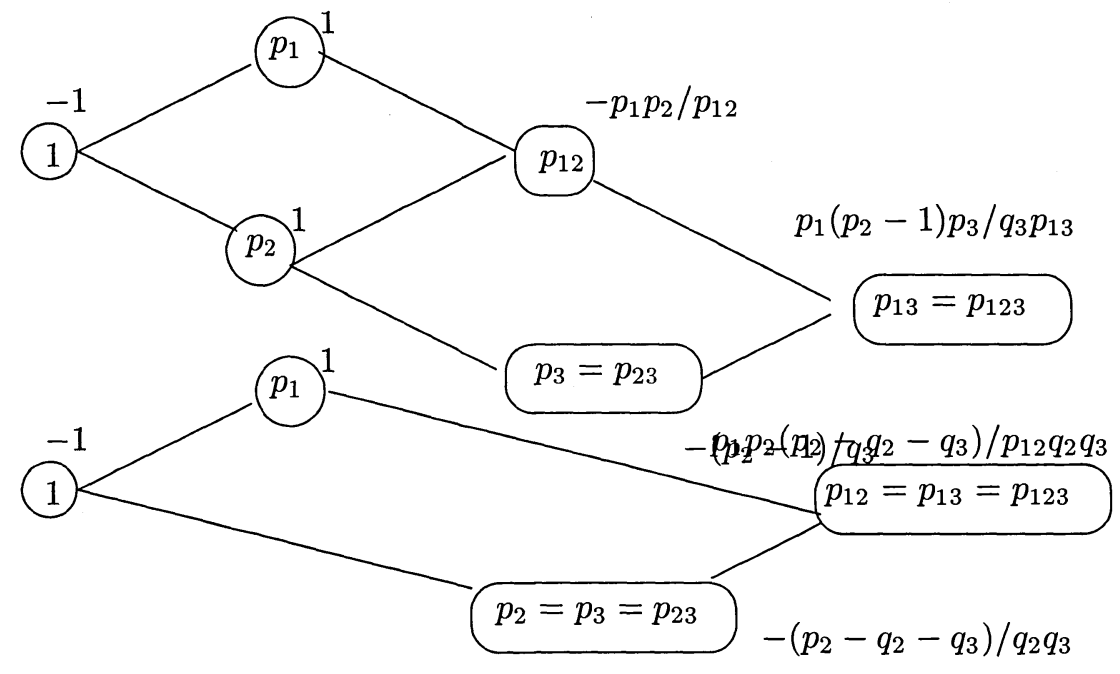

III.

IV.

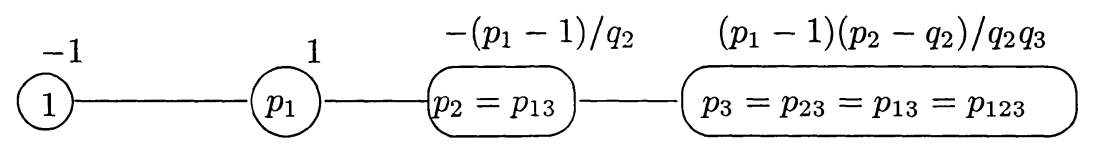

V.

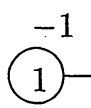

$$
(\mu+1) / h
$$$$
p_{1}=p_{2}=p_{3}=p_{12}=p_{23}=p_{13} \doteq p_{123}
$$ 

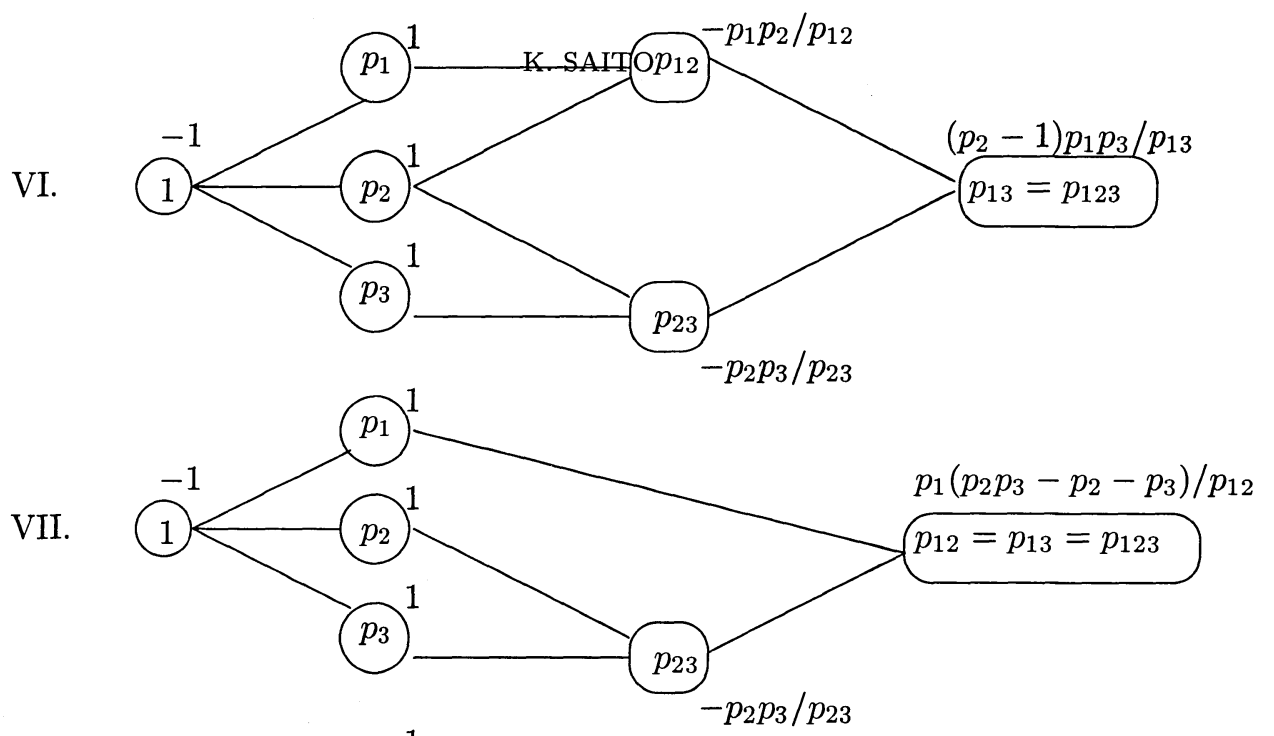

VI.
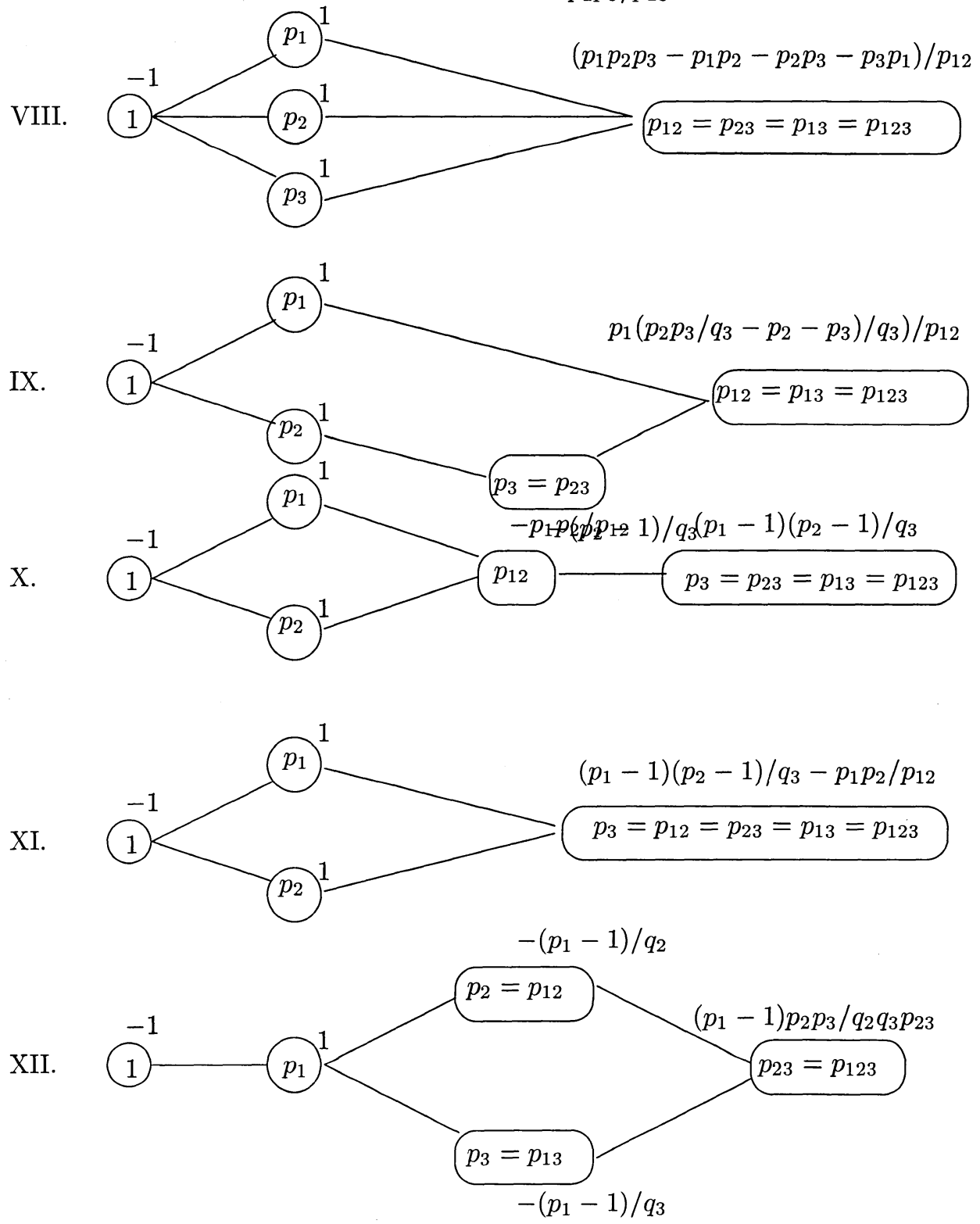
XIII.

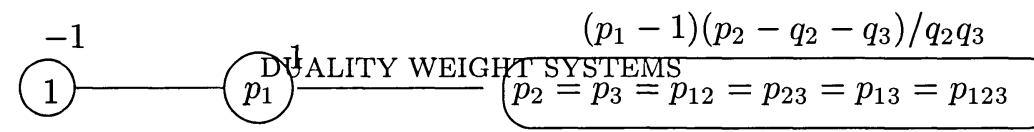
993

XIV.

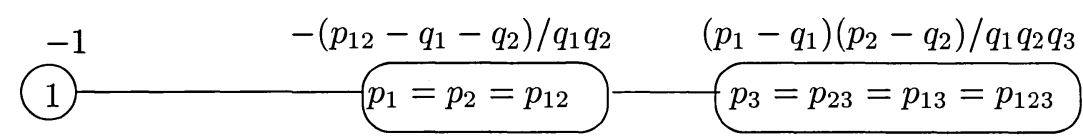

Proof of Theorem 5.2. The classification of the leveled posets is elementary and we give only an outline of the proof below. In fact, we classify leveled posets $(M(\underline{p}), n)$ generated by three integers $(\underline{p})=\left(p_{1}, p_{2}, p_{3}\right)$ with $p_{i} \geq 2$, where the level is defined by (5.1). First, we list up all division relations among $p_{1}, p_{2}$ and $p_{3}$, and then determine all isomorphism classes of $(M(\underline{p}), n)$ for each case (by examining the division relations among $p_{i j}$ 's and h). Up to a permutation of $p_{i}$ 's, we have the following cases.

I. There are no incidence relations among $p_{1}, p_{2}$ and $p_{3}$ and also among $p_{12}, p_{23}$ and $p_{31}$.

II. $p_{2} \neq p_{3}$ and there are no incidence relations between $p_{1}$ and $p_{2}$, and $p_{1}$ and $p_{3}$.

III. $p_{2}=p_{3}$ and there are no incidence relations among $p_{1}$ and $p_{2}=p_{3}$.

IV. $p_{1} \neq p_{2} \neq p_{3}(=h)$.

V. $p_{1}=p_{2}=p_{3}(=h)$

VI. There are no incidence relations among $p_{1}, p_{2}$ and $p_{3}$ and there are a relations $p_{13}=p_{123}=h$.

VII. There are no incidence relations among $p_{1}, p_{2}$ and $p_{3}$ and there are relations $p_{12}=p_{13}=p_{123}=h$.

VIII. There are no incidence relations among $p_{1}, p_{2}$ and $p_{3}$ and there are relations $p_{12}=p_{23}=p_{31}=p_{123}=h$.

IX. $p_{2} \neq p_{3}$, there are no incidence relations $p_{1}, p_{2}$ and $p_{3}$ and there is a relation $p_{3} \mid p_{12}$ so that $p_{12}=p_{13}=h$.

$\mathrm{X}$. There are no incidence relations among $p_{1}$ and $p_{2}$, and there is a relation $p_{12} \neq p_{3}$ and so $p_{3}=h$.

XI. There are no incidence relations between $p_{1}$ and $p_{2}$, and there is a relation $p_{12}=p_{3}=h$.

XII. $p_{1} \neq p_{2}$ and $p_{1} \neq p_{3}$, and there are no incidence relations among $p_{2}$ and $p_{3}$.

XIII. $p_{1} \neq p_{2}=p_{3}$.

XIV. $p_{1}=p_{2} \neq p_{3}$.

For any of the above 14 cases, the existence of a weight system $W$ belonging to the type is shown by the following two steps: i) take $(\underline{p})=\left(p_{1}, p_{2}, p_{3}\right)$ satisfying the conditions, ii) show that the weight system $W:=\left(p_{2} p_{3}, p_{3} p_{1}, p_{1} p_{2} ; p_{1} p_{2} p_{3}\right) / \operatorname{gcd}\left(p_{2} p_{3}, p_{3} p_{1}, p_{1} p_{2}\right)$ is regular and $M(p)=M(W)$.

We prepare a lemma for a calculation of the cyclotomic exponent.

ASSERTION 5.3. Let $W=(a, b, c ; h)$ be a weight system and $p_{1}, p_{2}, p_{3}, q_{1}, q_{2}$ and $q_{3}$ be integers introduced in (4.4).

i) If $a \mid(h-b)$, then $p_{2} \mid p_{1}$ and $q_{1} \mid\left(p_{2}-q_{2}\right)$.

ii) If $n\left(p_{1}\right)=1$, then $a \mid h, a \nmid b, a \nmid c$ and $q_{1}=1$.

iii) If $p_{3} \nmid p_{2}$, then $q_{2} \mid\left(p_{1}-q_{1}\right)$.

iv) If $n\left(p_{3}\right)=3\left(\Leftrightarrow p_{3}=h\right)$, then $(h, c)=1$ and $q_{3}=c$ divides either $p_{1}-q_{1}$ or $p_{2}-q_{2}$.

Proof. i) If $a \mid(h-b)$, then $(a, h) \mid b$ and hence $(a, h) \mid(b, h)$. This means $p_{2}=h /(h, b)$ divides $p_{1}=h /(h, a)$. Since $(a, h) q_{1} \mid(b, h)\left(p_{2}-q_{2}\right)$ and $q_{1}$ is prime to $(b, h) /(a, h)$, one has $q_{1} \mid\left(p_{2}-q_{2}\right)$. 
ii) If $n\left(p_{1}\right)=1$, then owing to i), neither $a \mid(h-b)$ nor $a \mid(h-c)$. So the only possibility is $a \mid(h-a)$. Then $a \nmid b$ otherwise $a \mid(h-b)$.

iii) Due to i), $b \nmid(h-c)$. So either $b \mid(h-b)$ or $b \mid(h-a)$. Hence either $b \mid h$ and hence $q_{2}=1$ for the first case, or $q_{2} \mid\left(p_{1}-q_{1}\right)$ for the latter case.

iv) The fact $p_{3}=h$ and $h / c=p_{3} / q_{3}$ imply iv).

We return to the proof of Theorem 5.2. The calculation of $e_{W}(\xi)$ is achieved by induction on the level $n(\xi)$. More precisely, it is achieved depending on the subposet $M_{\xi}:=\{\eta \in M(W): \eta \mid \xi\}$. The restriction of the Möbius function $\widetilde{\mu}(\cdot, \xi)$ of $M(W)$ to $M_{\xi}$ is the Möbius function of $M_{\xi}$.

Case $0 . \quad n(\xi)=0$.

In this case, $\xi=1$ and $M_{\xi}=1$. Formula (4.10) implies $1 \cdot e_{W}(1)=A(1) \tilde{\mu}(1,1)$ $=A(1)=-1(2.6)$, and therefore

$$
e_{W}(1)=-1=(-1)^{n(\xi)+1} .
$$

Case 1. $n(\xi)=1$.

By a permutation of the index, we may assume $\xi=p_{1}$ and $p_{2} \nmid \xi, p_{3} \nmid \xi$. In view of the Assertion $5.3 \mathrm{ii}$ ), one has $a \mid h$ and $\xi=p_{1}=h / a$. So $a \xi=h \equiv 0(h)$. On the other hand, $b \xi=h q_{2} \xi / p_{2} \not \equiv 0(h)$ and $c \xi=h q_{3} \xi / p_{3} \not \equiv 0(h)$. So $A(\xi)=h / a-1=p_{1}-1$ (2.5). The poset $M_{\xi}$ is equal to (1) $p_{1}$ and the Möbius function $\widetilde{\mu}(*, \xi)$ is ${ }^{-1}$ $\stackrel{1}{\circ}$. So (4.10) implies $\xi e_{W}(\xi)=A(\xi) \widetilde{\mu}\left(p_{1}, p_{1}\right)+A(1) \widetilde{\mu}\left(1, p_{1}\right)=\left(p_{1}-1\right)-(-1)=p_{1}$. Hence

$$
e_{W}(\xi)=1=(-1)^{n(\xi)+1}
$$

Case 2. $n(\xi)=2$.

By permuting the indices 1,2 and $3, M_{\xi}$ is one of the following 3 cases.

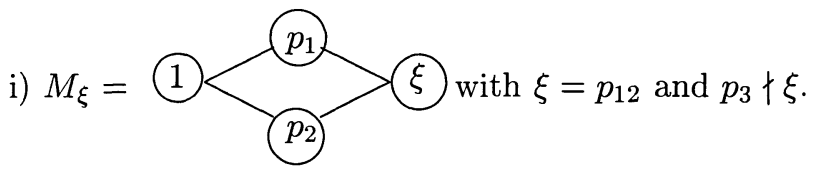

The Möbius function $\tilde{\mu}(*, \xi)$ is ${ }^{1}<_{\circ}^{\circ-1} \overbrace{-1}^{1}$. Since $p_{1}\left|\xi, p_{2}\right| \xi$ and $q_{1}=q_{2}=1$, one has $a \xi=h q_{1} \xi / p_{1} \equiv 0(h)$ and $b \xi=h q_{2} \xi / p_{2} \equiv 0(h)$. On the other hand, $p_{3} \nmid \xi$ and hence $c \xi=h q_{3} \xi / p_{3} \not \equiv 0(h)$. Thus $A(\xi)=-\left(p_{1}-1\right)\left(p_{2}-1\right)$ (2.5). So (4.10) implies $\xi e_{W}(\xi)=A(\xi) \widetilde{\mu}(\xi, \xi)+A\left(p_{1}\right) \widetilde{\mu}\left(p_{1}, \xi\right)+A\left(p_{2}\right) \widetilde{\mu}\left(p_{2}, \xi\right)+A(1) \widetilde{\mu}(1, \xi)=$ $-\left(p_{1}-1\right)\left(p_{2}-1\right)-\left(p_{1}-1\right)-\left(p_{2}-1\right)-1=-p_{1} p_{2}$. Hence

$$
e_{W}(\xi)=-p_{1} p_{2} / p_{12},
$$

whose sign is obviously equal to $(-1)^{n(\xi)+1}=-1$.

ii) $M_{\xi}=1$ ( 1 , with $\xi=p_{2}=p_{12}$ and $p_{3} \nmid \xi$.

The Möbius function $\tilde{\mu}(*, \xi)$ is $\stackrel{0}{\circ}-1 \quad \frac{1}{0}$. Due to Assertion 5.3 ii) and iii), $q_{1}=1, q_{2} \mid\left(p_{1}-1\right)$ and $A\left(p_{1}\right)=p_{1}-1, A\left(p_{2}\right)=-\left(p_{1}-1\right)\left(p_{2} / q_{2}-1\right)(2.5)$. Then 
(4.10) implies $\xi e_{W}(\xi)=A(\xi) \widetilde{\mu}(\xi, \xi)+A\left(p_{1}\right) \widetilde{\mu}\left(p_{1}, \xi\right)+A(1) \widetilde{\mu}(1, \xi)=-\left(p_{1}-1\right)\left(p_{2} / q_{2}-\right.$ 1) $-\left(p_{1}-1\right)-0=-\left(p_{1}-1\right) p_{2} / q_{2}$. Hence

$$
e_{W}(\xi)=-\left(p_{1}-1\right) / q_{2}
$$

whose sign is obviously equal to $(-1)^{n(\xi)+1}=-1$.

iii) $M_{\xi}=1$ with $\xi=p_{1}=p_{2}=p_{12}$ and $p_{3} \nmid \xi$.

The Möbius function $\widetilde{\mu}(*, \xi)$ is $\begin{gathered}-1 \\ 0\end{gathered}$ 1) $\left(p_{2} / q_{2}-1\right)(2.5)$. Since $p_{3} \nmid p_{1}=p_{2}$, the Assertion 5.3 iii) implies $q_{1} \mid p_{2}-q_{2}$ and $q_{2} \mid p_{1}-q_{1}$. The (4.10) implies $\xi e_{W}(\xi)=A(\xi) \widetilde{\mu}(\xi, \xi)+A(1) \tilde{\mu}(1, \xi)=-\left(p_{1} / q_{1}-\right.$ 1) $\left(p_{2} / q_{2}-1\right)+1=-\xi\left(\xi-q_{1}-q_{2}\right) /\left(q_{1} q_{2}\right)$. Hence

$$
e_{W}(\xi)=-\left(\xi-q_{1}-q_{2}\right) /\left(q_{1} q_{2}\right) .
$$

Let us show the negativity of this. Note that $W$ cannot be of type $A_{\ell}$, for $M\left(A_{\ell}\right)$ does not contain the sub-diagram $M_{\xi}$. Hence one has $p_{1} / q_{1} \geq 2$ and $p_{2} / q_{2} \geq 2$. So $\xi-q_{1}-q_{2} \geq 0$ and the equality holds only when $h / a=h / b=2$, which is the case of type $A_{\ell}$ and cannot occur.

Case 3. $n(\xi)=3$.

This is the case when $\xi=h=p_{123}$. By a use of formula (5.6) at the Remark 5.4.1., one may be able to reduce the proof of the positivity of $e_{W}(h)$ to the existence of the exponents prime to the Coxeter number which is readily proven in [S2, (2.2)] (quoted in (1.7)). But this is not sufficient for our purpose, since we need an exact expression of $e_{W}(h)$ for the later uses. So, we give here a direct proof of the positivity of $e_{W}(h)$.

There are 14 types of $M_{\xi}$ according to the table A. In all cases $A(h)=\left(p_{1} / q_{1}-\right.$ 1) $\left(p_{2} / q_{2}-1\right)\left(p_{3} / q_{3}-1\right)(2.5)$. Since the Möbius function depends on the type of $M_{\xi}$, we calculate $e_{W}(h)$ separately in each types.

I. Due to Assertion 5.3 ii), one has $q_{1}=q_{2}=q_{3}=1$.

The Möbius function $\widetilde{\mu}(*, h)$ is given by

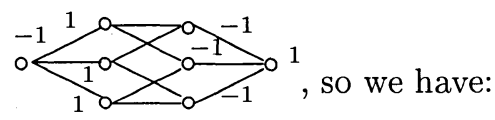

$$
\begin{aligned}
h e_{W}(h)= & \left(p_{1}-1\right)\left(p_{2}-1\right)\left(p_{3}-1\right)+\left(p_{1}-1\right)\left(p_{2}-1\right)+\left(p_{1}-1\right)\left(p_{3}-1\right) \\
& +\left(p_{2}-1\right)\left(p_{3}-1\right)+\left(p_{1}-1\right)+\left(p_{2}-1\right)+\left(p_{3}-1\right)+1 \\
= & p_{1} p_{2} p_{3} .
\end{aligned}
$$

Hence

$$
e_{W}(h)=p_{1} p_{2} p_{3} / p_{123},
$$

which is apparently positive.

II. Due to Assertions $5.3 \mathrm{ii})$ and iii), one has $q_{1}=q_{2}=1$ and $q_{3} \mid\left(p_{2}-1\right)$.

The Möbius function $\tilde{\mu}(*, h)$ is given by

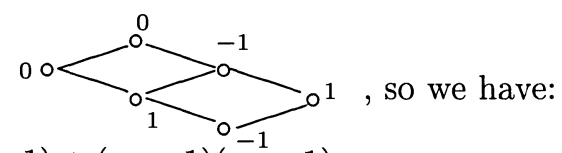

$$
\begin{aligned}
h e_{W}(h)= & \left(p_{1}-1\right)\left(p_{2}-1\right)\left(p_{3} / q_{3}-1\right)+\left(p_{1}-1\right)\left(p_{2}-1\right) \\
& +\left(p_{1}-1\right)\left(p_{3} / q_{3}-1\right)+\left(p_{2}-1\right)\left(p_{3} / q_{3}-1\right)+\left(p_{2}-1\right) \\
= & p_{1}\left(p_{2}-1\right) p_{3} / q_{3} .
\end{aligned}
$$


Hence

$$
e_{W}(h)=p_{1}\left(p_{2}-1\right) p_{3} /\left(p_{13} q_{3}\right)
$$

which is apparently positive for $p_{2} \geq 2(4.6)$.

III. Due to Assertion 5.3 ii) and iii), one has $q_{1}=1, q_{2} \mid\left(p_{2}-q_{3}\right)$ and $q_{3} \mid\left(p_{2}-q_{2}\right)$.

The Möbius function $\widetilde{\mu}(*, h)$ is given by

$$
\begin{aligned}
h e_{W}(h)= & \left(p_{1}-1\right)\left(p_{2} / q_{2}-1\right)\left(p_{3} / q_{3}-1\right)^{-1} \\
& +\left(p_{2} / q_{2}-1\right)\left(p_{3} / q_{3}-1\right)-\left(p_{1}-1\right)-1 \\
= & p_{1} p_{2}\left(p_{2}-q_{2}-q_{3}\right) /\left(q_{2} q_{3}\right) .
\end{aligned}
$$

Hence

$$
e_{W}(h)=p_{1} p_{2}\left(p_{2}-q_{2}-q_{3}\right) /\left(p_{12} q_{2} q_{3}\right) \text {. }
$$

The same argument for iii) of $n(\xi)=2$ shows the positivity $e_{W}(h)>0$.

IV. Due to Assertion 5.3 ii) and iii), and $q_{1}=1$ and $q_{2} \mid\left(p_{1}-q_{1}\right)$.

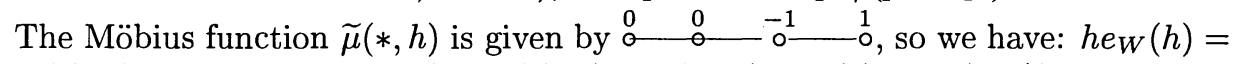
$\left(p_{1}-1\right)\left(p_{2} / q_{2}-1\right)\left(p_{3} / q_{3}-1\right)+\left(p_{1}-1\right)\left(p_{2} / q_{2}-1\right)=\left(p_{1}-1\right)\left(p_{2}-q_{2}\right) p_{3} /\left(q_{2} q_{3}\right)$. Hence

$$
e_{W}(h)=\left(p_{1}-1\right)\left(p_{2}-q_{2}\right) /\left(q_{2} q_{3}\right)
$$

which is apparently positive (see (4.6)).

$\mathrm{V}$. The Möbius function $\widetilde{\mu}(*, h)$ is given by $\stackrel{-1}{0}-1$, so we have: $h e_{W}(h)=$ $\left(p_{1} / q_{1}-1\right)\left(p_{2} / q_{2}-1\right)\left(p_{3} / q_{3}-1\right)+1=\mu_{W}+1$, where $\mu_{W}$ is the rank of the weight system $W(1.3)$. Hence

$$
e_{W}(h)=\left(\mu_{W}+1\right) / h,
$$

which is obviously positive. For later use, we describe $e_{W}(h)$ precisely. By definition, $a=q_{1}, b=q_{2}, c=q_{3}$ and $(a, h)=(b, h)=(c, h)=1$. These imply $(a, b)=(b, c)$ $=(c, a)=1$. We assume either i) $a|h-b, b| h-c$ and $c \mid h-a$, or ii) $a \mid h-b$, $b \mid h-a$ and $c \mid h-a$. Let us study each cases.

i) Put $k:=(h-b) / a, l:=(h-c) / b$, and $m:=(h-a) / c$. Solving this, we see

$$
(a, b, c ; h)=(l m-m+1, m k-k+1, k l-l+1 ; k l m+1) / d,
$$

where

$$
\begin{aligned}
d & :=(l m-m+1, k l m+1)=(m k-k+1, k l m+1) \\
& =(k l-l+1, k l m+1) \\
& =(l m-m+1, m k-k+1)=(m k-k+1, k l-l+1) \\
& =(k l-l+1, l m-m+1) .
\end{aligned}
$$

Then, since $\mu=(h-a)(h-b)(h-c) / a b c=k l m$, one has

$$
e_{W}(h)=(k l m+1) / h=d .
$$


ii) Put $k:=(h-b) / a, l:=(h-a) / b$. Solving this, we see

$$
(a, b, c ; h)=(l-1, k-1, d m ; k l-1) / d,
$$

where

$$
d:=(k-1, k l-1)=(l-1, k l-1)=(k-1, l-1)
$$

and $m$ is a divisor of $l$ such that $(m,(k-1) / d)=1$. Then one has

$$
e_{W}(h)=\left(\mu_{W}+1\right) / h=l k / m-d .
$$

VI. Due to Assertion 5.3 ii), one has $q_{1}=q_{2}=q_{3}=1$.

The Möbius function $\widetilde{\mu}(*, h)$ is given by

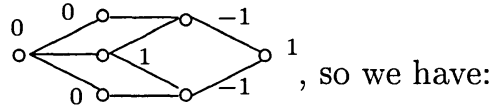

$$
\begin{aligned}
h e_{W}(h)= & \left(p_{1}-1\right)\left(p_{2}-1\right)\left(p_{3}-1\right) \\
& +\left(p_{1}-1\right)\left(p_{2}-1\right)+\left(p_{2}-1\right)\left(p_{3}-1\right)+\left(p_{2}-1\right) \\
= & \left(p_{2}-1\right) p_{1} p_{3} .
\end{aligned}
$$

Hence

$$
e_{W}(h)=\left(p_{2}-1\right) p_{1} p_{3} / p_{13},
$$

which is apparently positive.

VII. Due to Assertion 5.3 ii), one has $q_{1}=q_{2}=q_{3}=1$.

The Möbius function $\widetilde{\mu}(*, h)$ is given by

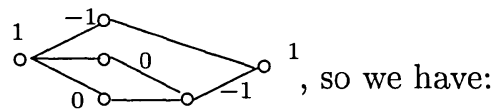

$$
\begin{aligned}
h e_{W}(h)= & \left(p_{1}-1\right)\left(p_{2}-1\right)\left(p_{3}-1\right) \\
& +\left(p_{2}-1\right)\left(p_{3}-1\right)-\left(p_{1}-1\right)-1 \\
= & p_{1}\left(p_{2} p_{3}-p_{2}-p_{3}\right) .
\end{aligned}
$$

Hence

$$
e_{W}(h)=p_{1}\left(p_{2} p_{3}-p_{2}-p_{3}\right) / p_{12}
$$

Since $p_{2}$ and $p_{3}$ are not less than $2, e_{W}(h)$ can be non-positive only when $p_{2}=p_{3}=2$, which contradicts to independence of $p_{1}$ and $p_{2}$.

VIII. Due to Assertion 5.3 ii), one has $q_{1}=q_{2}=q_{3}=1$.

The Möbius function $\tilde{\mu}(*, h)$ is given by

$$
\begin{aligned}
h e_{W}(h)= & \left(p_{1}-1\right)\left(p_{2}-1\right)\left(p_{3}-1\right) \\
& -\left(p_{1}-1\right)-\left(p_{2}-1\right)-\left(p_{3}-1\right)-2 \\
= & p_{1} p_{2} p_{3}-p_{1} p_{2}-p_{2} p_{3}-p_{1} p_{3} .
\end{aligned}
$$

Hence

$$
e_{W}(h)=\left(p_{1} p_{2} p_{3}-p_{1} p_{2}-p_{2} p_{3}-p_{1} p_{3}\right) / p_{12} .
$$


Positivity of $e_{W}(h)$ can be seen as follows. First, observe that $p_{i}$ is not a prime number. (If $p_{1}$ were prime, then the equality $p_{12}=p_{13}$ implies $p_{2}=p_{3}$. A contradiction!) By a permutation of indices we assume $p_{1}>p_{2}>p_{3} \geq 6$. Then $e_{W}(h)=\left(p_{1} p_{2} p_{3}-p_{1} p_{2}-\right.$ $\left.p_{2} p_{3}-p_{1} p_{3}\right) / p_{12}>\left(6 p_{1} p_{2}-p_{1} p_{2}-p_{1} p_{2}-p_{1} p_{2}\right) / p_{12} \geq 3$.

IX. Due to Assertion 5.3 ii) and iii), we have $q_{1}=q_{2}=1$ and $q_{3} \mid p_{2}-1$.

The Möbius function $\tilde{\mu}(*, h)$ is given by ${ }_{0}^{1}$

$$
\begin{aligned}
h e_{W}(h)= & \left(p_{1}-1\right)\left(p_{2}-1\right)\left(p_{3} / q_{3}-1\right) \\
& +\left(p_{2}-1\right)\left(p_{3} / q_{3}-1\right)-\left(p_{1}-1\right)-1 \\
= & p_{1}\left(p_{2} p_{3} / q_{3}-p_{2}-p_{3} / q_{3}\right) .
\end{aligned}
$$

Hence

$$
e_{W}(h)=p_{1}\left\{\left(p_{2}-1\right)\left(p_{3}-q_{3}\right) / q_{3}-1\right\} / p_{12} .
$$

The positivity of this can be shown as follows. At least $e_{W}(h)$ is non negative for (4.6). If it were zero, then $p_{2}-1=q_{3}, p_{3}-q_{3}=1$ and hence $p_{2}=p_{3}=2, q_{3}=1$. This contradicts the independence of $p_{2}$ and $p_{3}$.

$\mathrm{X}$. One has $a|h, b| h$ and $q_{1}=q_{2}=1$. Since $c=q_{3}$ divides either $h-a=a\left(p_{1}-1\right)$, $h-b=b\left(p_{2}-1\right)$ or $h-c$ and $(c, h)=1, q_{3}$ divides either $p_{1}-1$ or $p_{2}-1$.

The Möbius function $\tilde{\mu}(*, h)$ is given by

$$
\begin{aligned}
h e_{W}(h)= & \left(p_{1}-1\right)\left(p_{2}-1\right)\left(p_{3} / q_{3}-1\right) \\
& +\left(p_{1}-1\right)\left(p_{2}-1\right)-1 \\
= & \left(p_{1}-1\right)\left(p_{2}-1\right) p_{3} / q_{3} .
\end{aligned}
$$

Hence

$$
e_{W}(h)=\left(p_{1}-1\right)\left(p_{2}-1\right) / q_{3}
$$

which is non less than $\min \left\{p_{1}-1, p_{2}-1\right\}>0$.

XI. One has $a|h, b| h$ and $q_{1}=q_{2}=1$. $q_{3}$ divides either $p_{1}-1$ or $p_{2}-1$ as in type $X$.

The Möbius function $\tilde{\mu}(*, h)$ is given by ${ }_{-10}^{1}$, so we have

$$
\begin{aligned}
h e_{W}(h)= & \left(p_{1}-1\right)\left(p_{2}-1\right)\left(p_{3} / q_{3}-1\right) \\
& -\left(p_{1}-1\right)-\left(p_{2}-1\right)-1 \\
= & \left(p_{1}-1\right)\left(p_{2}-1\right) p_{3} / q_{3}-p_{1} p_{2} .
\end{aligned}
$$

Hence

$$
e_{W}(h)=\left(p_{1}-1\right)\left(p_{2}-1\right) / q_{3}-p_{1} p_{2} / p_{12} .
$$

The positivity of $e_{W}(h)$ can be seen as follows. First, we examine special case when one of $p_{1}$ and $p_{2}$ is equal to 2. Assume $p_{1}=2$. So $h=p_{12}=2 p_{2}$ and we have $e_{W}(h)=\left(p_{2}-1\right) / q_{3}-1$. There are two cases to consider: a) $q_{3} \mid\left(p_{1}-1\right)$. Then, 
$q_{3}=p_{1}-1=1$ and hence $e_{W}(h)=p_{2}-1-1$. This is non-positive only when $p_{2}=2$, which is impossible for $p_{1}=p_{2}$, b) $q_{3} \mid\left(p_{2}-1\right)$. Since $p_{3}=2 p_{2}$ is an even number, $q_{3}$ is odd. Since $p_{2}$ and $q_{3}$ are odd, $\left(p_{2}-1\right) / q_{3}$ is an even positive number $\geq 2$.

Next we consider the general case when $p_{1}, p_{2} \geq 3$. Let us show that $p_{3} / q_{3}>2$. Otherwise, $p_{3} / q_{3}=2$ and so $p_{3}=2, q_{3}=1$. Then $p_{1} \mid p_{3}=2$ and $p_{2} \mid p_{3}=2$ imply a contradiction: $p_{1}=p_{2}=p_{3}=2$. Assume $q_{3} \mid\left(p_{2}-1\right)$ and put $p_{2}-1=r q_{3}$. So

$$
\begin{aligned}
h e_{W}(h) & =\left(p_{1}-1\right) p_{3} r-p_{1}\left(r q_{3}+1\right)=r\left\{\left(p_{1}-1\right) p_{3}-p_{1} q_{3}\right\}-p_{1} \\
& \geq r\left\{\left(p_{1}-1\right) p_{3}-p_{1}\left(p_{3}-1\right) / 2\right\}-p_{1} \\
& =r\left(p_{1} / 2-1\right) p_{3}+(r / 2-1) p_{1} \\
& \geq p_{3} / 2-p_{1} / 2>0 .
\end{aligned}
$$

XII. One has $q_{1}=1, q_{2} \mid\left(p_{1}-1\right)$ and $q_{3} \mid p_{1}-1$.

The Möbius function $\tilde{\mu}(*, h)$ is given by ${ }^{2}$, so we have

$$
\begin{aligned}
h e_{W}(h)= & \left(p_{1}-1\right)\left(p_{2} / q_{2}-1\right)\left(p_{3} / q_{3}-1\right) \\
& +\left(p_{1}-1\right)\left(p_{2} / q_{2}-1\right)+\left(p_{1}-1\right)\left(p_{3} / q_{3}-1\right)+\left(p_{1}-1\right) \\
= & \left(p_{1}-1\right) p_{2} p_{3} /\left(q_{2} q_{3}\right) .
\end{aligned}
$$

Hence

$$
e_{W}(h)=\left(p_{1}-1\right) p_{2} p_{3} /\left(q_{2} q_{3} p_{23}\right),
$$

which is apparently positive.

XIII. One has $q_{1}=1$ and $h=p_{2}=p_{3}$.

The Möbius function $\widetilde{\mu}(*, h)$ is given by $\begin{array}{lll}0 & -1 & 1 \\ 0 & - & \end{array}$

$$
\begin{aligned}
h e_{W}(h) & =\left(p_{1}-1\right)\left(p_{2} / q_{2}-1\right)\left(p_{3} / q_{3}-1\right)-\left(p_{1}-1\right) \\
& =\left(p_{1}-1\right) p_{2}\left(p_{23}-q_{2}-q_{3}\right) /\left(q_{2} q_{3}\right) .
\end{aligned}
$$

Hence

$$
e_{W}(h)=\left(p_{1}-1\right)\left(p_{23}-q_{2}-q_{3}\right) /\left(q_{2} q_{3}\right) .
$$

Its positivity is shown by the same proof as in the case $n(\xi)=2 \mathrm{iii}$ ).

XIV. One has $h=p_{3}, q_{1} \mid p_{2}-q_{2}$ and $q_{2} \mid p_{1}-q_{1}$.

The Möbius function $\widetilde{\mu}(*, h)$ is given by $\stackrel{0}{0}-\frac{1}{0}-1$, so we have:

$$
\begin{aligned}
h e_{W}(h)= & \left(p_{1} / q_{1}-1\right)\left(p_{2} / q_{2}-1\right)\left(p_{3} / q_{3}-1\right) \\
& +\left(p_{1} / q_{1}-1\right)\left(p_{2} / q_{2}-1\right) \\
= & \left(p_{1}-q_{1}\right)\left(p_{2}-q_{2}\right) p_{3} /\left(q_{1} q_{2} q_{3}\right) .
\end{aligned}
$$

Hence

$$
e_{W}(h)=\left(p_{1}-q_{1}\right)\left(p_{2}-q_{2}\right) /\left(q_{1} q_{2} q_{3}\right),
$$

is apparently positive (cf. (4.6)).

These complete the proof of the Theorem 5.2. 
In the rest of $\S 5$, we discuss immediate consequences of Theorems 5.1-2.

1. Positivity of the multiplicity.

Recall the multiplicity (1.7) of a weight system $W: \operatorname{mult}(W):=a_{1}+a_{-1}=$ $\sharp\left\{i \in\{1, \ldots, \mu\} \mid m_{i} \in\{ \pm 1\}\right\}$. Since $a_{-1}=a_{h+1}$ by the duality (1.4), this is equal to the multiplicity of roots $\exp (2 \pi \sqrt{-1} / h)$ in the equation $\varphi_{W}(\lambda)=0(2.1)$. So, by definition of exponent $e_{W}(h)(3.1)$, one has

$$
e_{W}(h)=\operatorname{mult}(W) .
$$

Therefore, the positivity of $e_{W}(h)$ in the Theorem 5.1 is equivalent to that of mult $(W)$ and hence is equivalent to the existence of exponents prime to the Coxeter number $h$ (cf. Theorem 1.1). This fact for the classical cases $A_{l}, D_{l}$ and $E_{l}$ is well known ([Bo, Ch5 $\left.\left.\S 6 \mathrm{n}^{\circ} 2 \mathrm{Th} 1(1)\right]\right)$ and plays a basic role in the invariant theory for the classical root systems $[\mathrm{Sp}][\mathrm{S} 9]$.

2. Definition of the dual type posets.

Let us call a poset $(M(W), n)$ (but not $W$ ) of dual type, if there exists an involutive anti-automorphism $\iota$ of $M(W)$ (i.e. $\xi \prec \eta \Leftrightarrow \iota(\xi) \succ \iota(\eta)$, and $n(\xi)+n(\iota(\xi))=3$ for $\xi, \eta \in M(W))$. The next fact is an immediate consequence of the classification.

Fact. A leveled poset $(M(W), n)$ is of dual type, if and only if one has the equality:

$$
\sharp\{\xi \in M(W) \mid n(\xi) \text { is even }\}=\sharp\{\xi \in M(W) \mid n(\xi) \text { is odd }\} \text {. }
$$

In fact, the dual type posets are the types I, II, III, IV or V. An involution is often given by $\iota(\xi):=h / \xi$, but it is not always the case.

\section{Determination of $M(W)$ from $\varphi_{W}$.}

Fact. The characteristic polynomial $\varphi_{W}$ determines the leveled poset $(M(W), n)$ together with the generator $p_{1}, p_{2}$ and $p_{3}$.

A sketch of proof. The decomposition (3.1) gives the set $|M(W)|$ and the parity of the level $n(\xi)$ for $\xi \in|M(W)|$. Put $h:=\max \{|M(W)|\}$. Then, $n(\xi)=1$ for all odd parity elements except for $n(h)=3$, and $n(\xi)=2$ for all even parity elements except for $n(1)=0$. The generators $=\{\xi \mid n(\xi)=1\} \cup\left\{\xi \mid n(\xi)=2, \# M_{\xi} \leq 3\right\} \cup\{\xi \mid$ $\left.n(\xi)=3, \# M_{\xi} \leq 4\right\}$ with multiplicity $n(\xi)-\# M_{\xi}+2$ (except for the case XIV).

REMARK 5.4. 1. The characteristic polynomial $\varphi_{W}$ determines $W$ up to finite, as follows. Since $a_{i}=q_{i} h / p_{i}(i=1,2,3)$, one needs the data of $q_{i}(i=1,2,3)$ to recover the weights. If $n\left(p_{i}\right)=1$ then $q_{i}=1$. The formula of $e_{W}(\xi)$ in Table A together with its value determines $q_{i}$ except for the 2 cases: III. the equality $q_{2} q_{3} e_{W}\left(p_{2}=p_{3}\right)+p_{2}-q_{2}-q_{3}=0$ may have multi-solutions, and V (cf. Proof of Theorem 5.2 for the case $\mathrm{V}$ ).

2. The 14 types of posets are ordered according to their cardinality and degeneration relations:

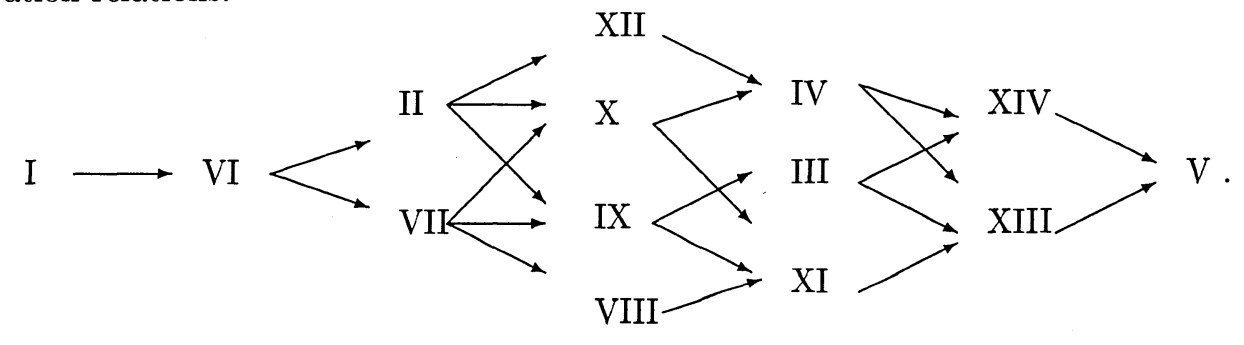




\section{Simplicity condition for the cyclotomic exponent.}

Definition 6.1. Let us call a weight system $W$ to be simple, if $e_{W}(\xi) \in\{ \pm 1\}$ for all $\xi \in M(W)$. In view of Theorem 5.11 , this is equivalent to:

$$
e_{W}(\xi)=(-1)^{n(\xi)+1}
$$

for all $\xi \in M(W)$. We give a necessary and sufficient condition for $W$ to be simple in the following Theorem 6.2 .

THEOREM 6.2. 1. A weight system $W$ is simple, if and only if the multiplicity $\operatorname{mult}(W)$ (see (1.7) and (5.6)) is equal to 1.

2. If mult $(W)=1$, then $M(W)$ is one of types I, II, III, IV, V or XI.

Proof. The condition mult $(W)=1$ is necessary for $W$ to be simple, because of the relation $e_{W}(h)=$ mult $(W)(5.6)$.

Assume mult $(W)=1$ and let us show that $e_{W}(\xi) \in\{ \pm 1\}$ for all $\xi \in M(W)$. If $n(\xi)=0$ or 1 , then $e_{W}(\xi)$ is automatically -1 or 1 , respectively (Theorem 5.12 ). If $n(\xi)=3$ then $\xi=h$ and $e_{W}(h)=\operatorname{mult}(W)=1$ by the assumption. Therefore we have only to show:

a) $e_{W}(\xi)=-1$ for $\xi \in M(W)$ with $n(\xi)=2$ assuming $e_{W}(h)=1$,

b) $e_{W}(h) \geq 2$ for any $W$ of non-dual type except for the type XI.

We use (5.4)-(5.5) and Table $A$ without referring to them explicitly.

Proof of a).

I. $e_{W}(h)=1$ implies $p_{1} p_{2} p_{3}=l c m\left(p_{1}, p_{2}, p_{3}\right)$. Hence, $p_{1}, p_{2}$ and $p_{3}$ are mutually prime. Therefore $e_{W}\left(p_{i j}\right)=-p_{i} p_{j} / p_{i j}=-1$ for $1 \leq i \neq j \leq 3$.

II. Since $\left(p_{2}-1\right) / q_{3}$ is an integer, $e_{W}(h)=\left(\left(p_{2}-1\right) / q_{3}\right)\left(p_{1} p_{3} / p_{13}\right)=1$ implies $\left(p_{2}-1\right) / q_{3}=1$ and $p_{1} p_{3} / p_{13}=1$. From the first equality, we obtain $e_{W}\left(p_{3}\right)=1$. From the latter equality, we obtain $\left(p_{1}, p_{3}\right)=1$. Since $p_{2} \mid p_{3}$, one has $\left(p_{1}, p_{2}\right)=1$ and $e_{W}\left(p_{12}\right)=-p_{1} p_{2} / p_{12}=-1$.

III. Since $\left(p_{2}-q_{2}-q_{3}\right) / q_{2} q_{3}$ is an integer, $e_{W}(h)=\left(p_{1} p_{2} / p_{12}\right)\left(\left(p_{2}-q_{2}-q_{3}\right) / q_{2} q_{3}\right)=$ 1 implies $-e_{W}\left(p_{23}\right)=\left(p_{2}-q_{2}-q_{3}\right) / q_{2} q_{3}=1$ and $p_{1} p_{2} / p_{12}=1$.

IV. $q_{1}=1$ and $h=a p_{1}$. So $p_{1} \mid p_{2}$ implies $b=(a, b) q_{2}$ and $p_{2}=p_{1} a /(a, b)$. Due to Assertion iii) and iv), one has $q_{2} \mid\left(p_{1}-1\right),(c, h)=1$ and $c=q_{3}$. Since $(a, b, c ; h)$ is regular, there are 3 cases to consider. i) $c \mid h$, ii) $c \mid(h-b)$, iii) $c \mid(h-a)$ and $(b, c) \mid h$.

i) $c=1$ and this case is included in ii). ii) $c \mid(h-b)=(h, b)\left(p_{2}-q_{2}\right)$ and hence $q_{3}=c \mid\left(p_{2}-q_{2}\right)$. Then $e_{W}(h)=\left(\left(p_{1}-1\right) / q_{2}\right)\left(\left(p_{2}-q_{2}\right) / q_{3}\right)=1$ implies $1=\left(p_{1}-1\right) / q_{2}=-e\left(p_{2}\right)$. iii) $c \mid(h-a)=a\left(p_{1}-1\right)$. Since $(c, h)=\left(q_{3}, p_{3}\right)=1$, so $(c, a)=1$ and therefore $q_{3} \mid\left(p_{1}-1\right)$. On the other hand $\left(q_{2}, q_{3}\right)=1$ (4.8) together with $e_{W}(h)=\left(\left(p_{1}-1\right) / q_{2} q_{3}\right)\left(p_{2}-q_{2}\right)=1$ implies $\left(p_{1}-1\right) / q_{2} q_{3}=1$ and $p_{2}-q_{2}=1$. The second equality together with the general fact $2 \leq p_{2} / q_{2}$ implies $p_{2}=2$ and $q_{2}=1$. Then $2 \leq p_{1}<p_{2}=2$ is impossible so that this case cannot occur.

$\mathrm{V}$. There is no element $\xi \in M(W)$ with $n(\xi)=2$.

Proof of b).

VI. Suppose $e_{W}(h)=1$. Then $e_{W}(h) \geq p_{2}-1$ implies $p_{2}=2$. This is impossible, since $p_{2} \mid p_{13}$ implies either $p_{2} \mid p_{1}$ or $p_{2} \mid p_{3}$, which contradicts the incidencialy independence of $p_{2}$ from $p_{1}$ and $p_{3}$.

VII. First note that $p_{1} p_{2}>p_{12}$, else $p_{1} p_{2}=p_{12}=p_{13}=p_{1} p_{3} /\left(p_{1}, p_{3}\right)$ implies $p_{2} \mid p_{3}$ : a contradiction. For the same reason, $p_{1} p_{3}>p_{13}$. These imply that $p_{2}$ and $p_{3}$ 
cannot be prime numbers. (If $p_{2}$ were a prime, then $p_{1} p_{2}>p_{12}$ implies $p_{2} \mid p_{1}$.) So we have $p_{2}, p_{3} \geq 4$. One has $e_{W}(h)=p_{1} p_{2} / p_{12}+p_{1} p_{3} / p_{13}+p_{1}\left\{\left(p_{2}-2\right)\left(p_{3}-2\right)-2\right\} / p_{12} \geq$ $p_{1} p_{2} / p_{12}+p_{1} p_{3} / p_{13} \geq 4$.

VIII. Observe that any of $p_{i}$ is not a prime number. (Suppose $p_{1}$ were a prime. Then $p_{12}=p_{1} p_{2}$ equals $p_{13}=p_{1} p_{3}$ implies $p_{2}=p_{3}$. A contradiction!). By permutation of indices we may assume $p_{1}>p_{2}>p_{3} \geq 6$. Then $e_{W}(h)=\left(p_{1} p_{2} p_{3}-p_{1} p_{2}-\right.$ $\left.p_{2} p_{3}-p_{1} p_{3}\right) / p_{12}>\left(6 p_{1} p_{2}-p_{1} p_{2}-p_{1} p_{2}-p_{1} p_{2}\right) / p_{12} \geq 3$.

IX. Since $p_{12}=p_{1} p_{2} /\left(p_{1}, p_{2}\right)$, the formula for $e_{W}(h)$ is rewritten as $e_{W}(h)=$ $\left(p_{1}, p_{2}\right)\left\{p_{3} / q_{3}\left(1-1 / p_{2}\right)-1\right\}$. Suppose $\left(p_{1}, p_{2}\right) \neq 1$. Then substituting $\left(p_{1}, p_{2}\right) \geq$ $2, p_{2} \geq 4$ and $p_{3} / q_{3} \geq 2$ in the expression of $e_{W}(h)$, one obtains $e_{W}(h) \geq 1$ and the equality $e_{W}(h)=1$ occurs only when $p_{2}=4$ and $p_{3} / q_{3}=2$. Since $\left(p_{3}, q_{3}\right)=1$, this implies $q_{3}=1$ and $p_{3}=2$, which contradicts $p_{2} \mid p_{3}$. Therefore we have $\left(p_{1}, p_{2}\right)=1$. Then $p_{1} p_{2}=p_{12}=p_{13}=p_{1} p_{3} /\left(p_{1}, p_{3}\right)$ and hence $p_{3}=p_{2}\left(p_{1}, p_{3}\right)$. So we have $e_{W}(h)=\left(p_{2}-1\right)\left(p_{1}, p_{3}\right) / q_{3}-1$. Since $p_{2} \neq p_{3}$, we have $\left(p_{1}, p_{3}\right) \geq 2$. Therefore $e_{W}(h)=1$ implies $\left(p_{2}-1\right) / q_{3}=1$ and $\left(p_{1}, p_{3}\right)=2$. But this gives a contradiction, for $\left(p_{1}, p_{3}\right)=2$ implies that $p_{1}$ and $p_{3}$ are even and hence $\left(p_{1}, p_{2}\right)=1$ implies $p_{2}$ is odd. Then $q_{2}=p_{2}-1$ is even and hence $\left(p_{2}, q_{2}\right)$ contains a factor $2 \neq 1$.

X. Suppose $e_{W}(h)=1$. Since $q_{3}$ divides either $p_{1}-1$ or $p_{2}-1$, assume $q_{3} \mid\left(p_{1}-1\right)$. Then $\left(\left(p_{1}-1\right) / q_{3}\right)\left(p_{2}-1\right)=1$ implies $q_{3}=p_{1}-1$ and $p_{2}=2$. Since $p_{1}$ and $p_{2}$ are independent, $p_{1}$ is odd. This is a contradiction, since $p_{2} \mid p_{3}$ implies $p_{3}$ is even and $q_{3}=p_{1}-1$ implies $q_{3}$ is even, contradicting $\left(p_{3}, q_{3}\right)=1$.

XI. There is no element $\xi \in M(W)$ with $n(\xi)=2$.

XII. Similar to IV, one has $q_{1}=1, h=p_{1} a, b=q_{2}(a, b), p_{2}=p_{1} a /(a, b), c=$ $q_{3}(a, c), p_{3}=p_{1} a /(a, c)$, and $q_{2}, q_{3} \mid\left(p_{1}-1\right)$. The fact $\operatorname{lcm}\left(p_{1}, p_{2}, p_{3}\right)=h \mathrm{im}$ plies $a=l c m(a /(a, b), a /(a, c))$ and hence $\operatorname{gcd}((a, b),(a, c))=1$. Then $p_{2} p_{3} / p_{23}=$ $p_{1} a /(a, b)(a, c)$, where the factor $a /(a, b)(a, c)$ is an integer. Thus $e_{W}(h)=\left(p_{1}-\right.$ 1) $p_{2} p_{3} / q_{2} q_{3} p_{23}=\left\{\left(p_{1}-1\right) a /(a, b)(a, c)\right\} p_{1} / q_{2} q_{3}$, where the factor $\{*\}$ is an integer and the denominator $q_{2} q_{3}$ is prime to $p_{1}$. Then the fact $e_{W}(h)$ is an integer implies that $q_{2} q_{3}$ divides the factor $\{*\}$ and that $e_{W}(h)$ is a multiple of $p_{1} \geq 2$.

XIII. There is no element $\xi \in M(W)$ with $n(\xi)=2$. Nevertheless, we show $e_{W}(h) \geq 2$. In this case, $q_{1}=1$ and $h=p_{1} a$. Since $h=p_{2}=p_{3}$, one gets $q_{2}=b$ and $q_{3}=c . \quad\left(q_{2}, p_{2}\right)=\left(q_{3}, p_{3}\right)=1$ imply $\left(q_{2}, p_{1} a\right)=\left(q_{3}, p_{1} a\right)=1$. $(b, c) \mid h$ implies $\left(q_{2}, q_{3}\right)=1$. Consider the following cases: i) $b \mid(h-1)$ and $c \mid(h-a)$, ii) $b \mid(h-a)$ and $c \mid(h-b)$, iii) $b \mid(h-a)$ and $c \mid(h-c)$, iv $) b \mid(h-b)$ and $c \mid(h-b), \mathrm{v}) b \mid(h-b)$ and $c \mid(h-c)$, vi) $b \mid(h-c)$ and $c \mid(h-b)$.

i) $q_{2} \mid\left(p_{1}-1\right)$ and $q_{3} \mid\left(p_{1}-1\right)$, so $\left(q_{2}, q_{3}\right)=1$ implies $q_{2} q_{3} \mid\left(p_{1}-1\right)$. Hence $p_{1}-q_{2}-q_{3} \geq q_{2} q_{3}-q_{2}-q_{3}+1 \geq\left(q_{2}-1\right)\left(q_{3}-1\right) \geq 0$ and $e_{W}(h)=\left(\left(p_{1}-1\right) / q_{2} q_{3}\right)\left(p_{2}-\right.$ $\left.q_{2}-q_{3}\right) \geq 1 \cdot\left(2 p_{1}-q_{2}-q_{3}\right) \geq p_{1} \geq 2$.

ii) $q_{2} \mid\left(p_{1}-1\right)$ and $q_{3} \mid\left(p_{2}-q_{2}\right)$. So $e_{W}(h)=\left(\left(p_{1}-1\right) / q_{2}\right)\left(\left(p_{2}-q_{2}-q_{3}\right) / q_{3}\right)=1$ implies $p_{1}-1=q_{2}$ and $p_{2}-q_{2}=2 q_{3}$. This is impossible, since the last equality implies that $p_{2}$ and $q_{2}$ are simultaneously odd and hence $p_{1}=1+q_{2}$ is even. This contradicts $p_{1} \mid p_{2}$.

iii) $q_{2} \mid\left(p_{1}-1\right)$ and $q_{3} \mid h$, so $q_{3}=1$. Then $e_{W}(h) \geq 2$ can be shown similar to the case i).

iv) $q_{2} \mid h$ and $q_{3} \mid\left(p_{1} a-q_{2}\right)$, so $q_{2}=1$. Then $e_{W}(h) \geq 2$ can be shown similar to the case ii). 
v) $q_{2} \mid h$ and $q_{3} \mid h$, so $q_{2}=q_{3}=1$. Then $e_{W}(h) \geq 2$ can be shown similar to the case i).

vi) $q_{2} \mid\left(p_{1} a-q_{3}\right)$ and $q_{3} \mid p_{1} a-q_{2}$. Put $p_{1} a-q_{3}=u q_{2}$ and $p_{1} a-q_{2}=v q_{3}$. Then $(u-1) q_{2}=(v-1) q_{3}$ and $\left(q_{2}, q_{3}\right)=1$ implies the existence of $r>0$ such that $u-1=r q_{3}, v-1=r q_{2}$ and $p_{1} a=r q_{2} q_{3}+q_{2}+q_{3}$. Thus, $e_{W}(h)=\left(p_{1}-1\right) r$. Therefore $e_{W}(h)=1$ implies $p_{1}=2$ and $r=1$. Then $p_{1} a+1=\left(q_{2}+1\right)\left(q_{3}+1\right)$ and $p_{1}=2$ imply that $q_{2}$ and $q_{3}$ are even numbers contradicting $\left(q_{2}, q_{3}\right)=1$.

XIV. Put $p:=p_{1}=p_{2}$ and $d=(h, a)=(h, b)$ so that $h=p d, a=q_{1} d, b=q_{2} d$ and $\left(p, q_{1}\right)=\left(p, q_{2}\right)=1$. One has $p_{3}=h=p d, q_{3}=c$ and $\left(q_{3}, p d\right)=1$. The regularity of the weight system implies that $a, b$ and $c$ divide either $h-a$ or $h-b$. Because of the symmetry among $a$ and $b$, we consider only the following 4 cases: i) $a|(h-a), b|(h-b), c \mid(h-a)$, ii) $a|(h-a), b|(h-a), c \mid(h-a)$, iii) $a|(h-a), b|(h-a), c \mid(h-b)$, iv $) a|(h-b), b|(h-b), c \mid(h-a)$.

i) $q_{1}=q_{2}=1$ and $q_{3} \mid(p-1) d$ and hence $q_{3} \mid p-1$. Then $e_{W}(h)=(p-1)(p-$ 1) $/ q_{3}=1$ implies $p-1=1$ and $(p-1) / q_{3}=1$. So $p=2$ and $q_{1}=q_{2}=q_{3}=1$ and hence $W=(d, d, 1 ; 2 d)$ is of type $A_{\ell}$, which is excluded from the consideration.

ii) $q_{1}=1, q_{2} \mid p-1$ and $q_{3} \mid(p-1) d$ and so $q_{3} \mid p-1$. Since $(b, c)=\left(q_{2}, q_{3}\right)$ divides $h=p d$ and $\left(q_{2}, p\right)=\left(q_{3}, d\right)=1$, one has $\left(q_{2}, q_{3}\right)=1$. Thus $e_{W}(h)=$ $\left((p-1) / q_{2} q_{3}\right)\left(p-q_{2}\right)=1$ implies $p-1=q_{2} q_{3}$ and $p-q_{2}=1$. For $p / q_{2} \geq 2$, this is possible only when $q_{2}=1$ and $p=2$ and therefore $q_{2}=q_{3}=1$. This case is excluded as type $A_{\ell}$ as in the case i).

iii) $q_{1}=1, q_{2} \mid p_{1}-1$ and $q_{3} \mid p_{2}-q_{2}$. Thus $e_{W}(h)=\left((p-1) / q_{2}\right)\left(\left(p-q_{2}\right) / q_{3}\right)=1$ implies $p-1=q_{2}$. Again $p / q_{2} \geq 2$ implies $p=2$ and $q_{1}=q_{2}=1$. So $W=(d, d, 1 ; 2 d)$ is of type $A_{\ell}$ and is excluded.

iv) $q_{1}\left|\left(p-q_{2}\right), q_{2}\right|\left(p-q_{1}\right)$ and $q_{3} \mid\left(p-q_{1}\right)$. Since $(b, c)=\left(q_{2}, q_{3}\right)$ divides $h$ and $\left(q_{3}, h\right)=1$, one has $\left(q_{2}, q_{3}\right)=1$. Thus $e_{W}(h)=\left(\left(p-q_{1}\right) / q_{2} q_{3}\right)\left(\left(p-q_{2}\right) / q_{1}\right)=1$ implies $p-q_{1}=q_{2} q_{3}$ and $p-q_{2}=q_{1}$. This implies $q_{3}=1$. Together with the fact $p / q_{1}, p / q_{2} \geq 2$ one has $p=2$ and $q_{1}=q_{2}=1$. So $W=(d, d, 1 ; 2 d)$ is of type $A_{\ell}$ and is excluded.

These complete a proof of Theorem 6.2.

Some additional calculations to the proof of Theorem 6.2 enable to list up all simple weight systems. Precisely, let us call a poset $M(\underline{p})$ generated by three integers $(\underline{p})=\left(p_{1}, p_{2}, p_{3}\right)$ simple, if there exists a weight system $W$ of multiplicity 1 such that $\bar{M}(\underline{p})=M(W)$, and call $W$ a (simple) weight system representing $M(\underline{p})$. Then the next theorem gives a list of all simple posets together with a list of all weight systems representing them. For a relation with geometry, see Corollary 10.22 and Remark 10.33 .

THEOREM 6.3. Consider the poset $M(\underline{p})$ generated by three integers $(\underline{p})=$ $\left(p_{1}, p_{2}, p_{3}\right)$ with $p_{i} \geq 2$. For each type of $M(\underline{p})$, we give a list of:

i) The arithmetic conditions for $M(\underline{p})$ to be simple,

ii) The list of simple weight systems $W$ representing $M(\underline{p})$,

iii) The rank $\mu_{W}$ of weight systems $W$ representing $M(\underline{p})$.

I. The poset $M(\underline{p})$ is simple of type $I$, if and only if

$$
\left(p_{i}, p_{j}\right)=1
$$

for $i j=1,2,3$ and $i \neq j$. The simple weight system representing $M(\underline{p})$ is unique and is given by

$$
W=\left(p_{1} p_{2}, p_{2} p_{3}, p_{3} p_{1} ; p_{1} p_{2} p_{3}\right)
$$




$$
\mu_{W}=\left(p_{1}-1\right)\left(p_{2}-1\right)\left(p_{3}-1\right) .
$$

II. The poset $M(\underline{p})$ is simple of type II, if and only if

$$
p_{2} \neq p_{3}, \quad\left(p_{1}, p_{3}\right)=1 \quad \text { and } \quad\left(p_{2}-1, p_{3}\right)=1 .
$$

The simple weight system representing $M(\underline{p})$ is uniquely given by

$$
\begin{gathered}
W=\left(p_{3}, p_{1} p_{3} / p_{2},\left(p_{2}-1\right) p_{1} ; p_{1} p_{3}\right) \\
\mu_{W}=\left(p_{1}-1\right)\left(p_{3}-p_{2}+1\right) .
\end{gathered}
$$

III. The poset $M(\underline{p})$ is simple of type III, if and only if

$$
p_{2}=p_{3} \quad \text { and } \quad\left(p_{1}, p_{2}\right)=1 .
$$

The simple weight system representing $M(\underline{p})$ is given by

$$
W=\left(p_{2}, p_{1} q_{2}, p_{1} q_{3} ; p_{1} p_{2}\right)
$$

for any positive integers $q_{2}$ and $q_{3}$ such that $p_{2}+1=\left(q_{2}+1\right)\left(q_{3}+1\right)$ and $\left(q_{2}, q_{3}\right)=1$.

$$
\mu_{W}=\left(p_{1}-1\right)\left(p_{2}+1\right) \text {. }
$$

IV. The poset $M(\underline{p})$ is simple of type IV, if and only if

$$
p_{2} \neq p_{2} \neq p_{3}, \quad\left(p_{1}-1, p_{2}\right)=1 \quad \text { and } \quad\left(p_{2}-p_{1}+1, p_{3}\right)=1 \text {. }
$$

The simple weight system representing $M(\underline{p})$ is uniquely given by

$$
\begin{gathered}
W=\left(p_{3} / p_{1},\left(p_{1}-1\right) p_{3} / p_{2}, p_{2}-p_{1}+1 ; p_{3}\right) \\
\mu_{W}=p_{3}-p_{2}+p_{1}-1 .
\end{gathered}
$$

$\mathrm{V}$. The poset $M(\underline{p})$ is simple of type $\mathrm{V}$, if and only if

$$
p_{1}=p_{2}=p_{3}=: h \text {. }
$$

The simple weight system representing $M(\underline{p})$ is either type $A_{h-1}$ or

$$
W=(l m-m+1, m k-k+1, k l-1+1 ; h)
$$

for positive integers $k, l$ and $m$ with $k l m=h-1$ and $(\operatorname{lm}-m+1, h)=1$.

$$
\mu_{W}=h-1 \text {. }
$$

VI, VII, VIII, IX, X, XII, XIII or XIV. The poset $M(\underline{p})$ cannot be simple.

XI. The poset $M(\underline{p})$ is simple of type XI, if and only if $\left(p_{1}, p_{2}, p_{3}\right)$ belongs to one of the following three series:

$$
\begin{aligned}
& p_{1}=2, \quad p_{2}=4 k+3, \quad p_{3}=2(4 k+3) \quad \text { for } \quad k \in \mathbb{Z}_{\geq 0}, \\
& p_{1}=3, \quad p_{2}=3 k+2, \quad p_{3}=3(3 k+2) \quad \text { for } \quad k \in \mathbb{Z}_{\geq 0}, \\
& p_{1}=4, \quad p_{2}=2(2 k+1), \quad p_{3}=4(2 k+1) \quad \text { for } \quad k \in \mathbb{Z}_{>0} .
\end{aligned}
$$


Then the simple weight system representing $M(\underline{p})$ is uniquely given by

$$
W=\left(p_{3} / p_{1}, p_{3} / p_{2}, q_{3} ; p_{3}\right)
$$

for $q_{3}=2 k+1,3 k+1$, and $4 k+1$, respectively. The rank $\mu_{W}$ is given by

$$
\mu_{W}=p_{1}+p_{2}+p_{3}-1 \text {. }
$$

According to the series (6.18), (6.19) and (6.20), the representing weight system $W$, its rank $\mu_{W}$ and its smallest exponent $\varepsilon_{W}$ are given by

$$
\begin{aligned}
& W=(4 k+3,2,2 k+1 ; 2(4 k+3)), \\
& \mu_{W}=12 k+10, \quad \varepsilon_{W}=-2 k, \quad \nu_{W}=4 k \quad \text { and } a_{0}=2, \quad \text { for } k \in \mathbb{Z}_{\geq 0}, \\
& W=(3 k+2,3,3 k+1 ; 3(3 k+2)), \\
& \mu_{W}=12 k+10, \quad \varepsilon_{W}=-3 k, \quad \nu_{W}=6 k \quad \text { and } a_{0}=2, \quad \text { for } k \in \mathbb{Z}_{\geq 0} \\
& W=(2 k+1,2,4 k+1 ; 4(2 k+1)), \\
& \mu_{W}=12 k+9, \quad \varepsilon_{W}=-2 k, \quad \nu_{W}=6 k \quad \text { and } a_{0}=2, \text { for } k \in \mathbb{Z}_{>0}
\end{aligned}
$$

Proof. I. Simplicity of $W$ implies $p_{1} p_{2} p_{3}=p_{123}$. Since $q_{1}=q_{2}=q_{3}=1$, weights $a=h / p_{1}, b=h / p_{2}, c=h / p_{3}$ are uniquely determined.

II. Simplicity of $W$ implies $q_{3}=p_{2}-1, p_{1} p_{2}=p_{12}$ and $p_{1} p_{3}=p_{123}$, so $\left(p_{1}, p_{3}\right)=$ $\left(p_{1}, p_{2}\right)=1$ and $\left(p_{2}-1, p_{3}\right)=1$. Since $q_{1}=q_{2}=1$ and $q_{3}=p_{2}-1$, the representing weights system is given by $a=h / p_{1}=p_{3}, b=h / p_{2}=p_{1} p_{3} / p_{2}$ and $c=\left(p_{2}-1\right) h / p_{3}=$ $\left(p_{2}-1\right) p_{1}$. Conversely, for given $\underline{p}$ with $p_{2} \neq \underline{p}_{3}$, put $q_{3}:=p_{2}-1$. Then one has $\left(q_{3}, p_{3}\right)=\left(p_{2}-1, p_{3}\right)=1$ and $q_{3} / p_{3}=\left(p_{2}-1\right) / p_{3}<p_{2} / p_{3} \leq 1 / 2$.

III. Simplicity of $W$ implies $p_{2}=q_{2} q_{3}+q_{2}+q_{3}$ and $p_{1} p_{2}=p_{12}(=h)$. Conversely, for a given $p$ with the conditions, any positive integral solution $q_{2}, q_{3}$ of the equality $p_{2}+1=\left(q_{2}+1\right)\left(q_{3}+1\right)$ with $\left(q_{2}, q_{3}\right)=1$ satisfies $\left(p_{2}, q_{2}\right)=\left(p_{3}, q_{3}\right)=1$ and the inequality $q_{2} / p_{2} \leq\left(q_{2}+1\right) /\left(p_{2}+1\right)=1 /\left(q_{3}+1\right) \leq 1 / 2$ and the inequality $q_{3} / p_{3} \leq 1 / 2$.

IV. Simplicity of $W$ implies $q_{2}=p_{1}-1$ and $q_{3}=p_{2}-q_{2}=p_{2}-p_{1}+1$. Since $q_{1}=1$, the weights are given by $a=h / p_{1}, b=q_{2} h / p_{2}=\left(p_{1}-1\right) h / p_{2}$ and $c=q_{3} h / p_{3}=$ $p_{2}-p_{1}+1$. Conversely, for a given $p$ with the conditions, put $q_{1}=1, q_{2}=p_{1}-1$ and $q_{3}=p_{2}-p_{1}+1$. Then $\left(q_{2}, p_{2}\right)=1,\left(q_{3}, p_{3}\right)=1$ and $q_{2} / p_{2}=\left(p_{1}-1\right) / p_{2}<p_{1} / p_{2} \leq$ $1 / 2, q_{3} / p_{3}=\left(p_{2}-p_{1}+1\right) / p_{3}<p_{2} / p_{3} \leq 1 / 2$.

$\mathrm{V}$. Recall the descriptions i) and ii) of the weight system of type $\mathrm{V}$ in the proof of Theorem 5.1. The case i) yields the result. The case ii) reduces to the type $A_{\ell}$, since $e_{W}(h)=1$ implies $1=1^{\prime}$ and $k-1=d$.

XI. Since $n\left(p_{1}\right)=n\left(p_{2}\right)=1$ and $n\left(p_{3}\right)=3$, one has $q_{1}=q_{2}=1$ and $q_{3}=c$ (Assertion 5.3). This implies (6.20). The (6.21) follows from $h e_{W}(h)=\mu-\left(p_{1}-1\right)-$ $\left(p_{2}-1\right)-1$ by putting $e_{W}(h)=1$.

We determine the set $p_{1}, p_{2}, p_{3}$ and $q_{3}$. In the proof of Theorem 5.1 , we have shown that $p_{3} / q_{3}>2$. Since $q_{3}$ divides either $p_{1}-1$ or $p_{2}-1$, we may assume $q_{3} \mid p_{2}-1$ and put $p_{2}-1=r q_{3}$. So $h e_{W}(h)=r\left\{\left(p_{1}-1\right) p_{3}-p_{1} q_{3}\right\}-p_{1}$.

If $p_{1} \geq 5$, then

$$
h e_{W}(h) \geq r\left(4 p_{3}-5 q_{3}\right)-5=h+r\left(p_{3}-2 q_{3}\right) 5 / 2+(r 3-2) p_{3} / 2-5 .
$$

Since $p_{3}>2 q_{3}$, the second term is positive. Since $r 3-2 \geq 1$ and $p_{3} / 2 \geq p_{1} \geq 5$ the sum of the last two terms is non negative. This means $e_{W}(h)>1$. In the other words, if $e_{W}(h)=1$, then $p_{2}$ is either 2,3 , or 4 . 
Case $p_{1}=2$. Then $h=p_{3}=2 p_{2}$ and $p_{2}$ is odd number. Then $\left(p_{3}, q_{3}\right)=1$ implies $q_{3}$ is odd so that $r$ is a multiple of 2 . On the other hand, $h e_{W}(h)=r\left\{p_{3}-2 q_{3}\right\}-2$ and hence $e_{W}(h)=r\left(1-q_{3} /\left(r q_{3}+\right)\right)-1 /\left(r q_{3}+1\right)>r-1-1 /\left(r q_{3}+1\right)$. Therefore $e_{W}(h)=1$ implies $r \leq 2$ and so $r=2$. Then, we have

$$
p_{1}=2, p_{2}=2 q_{3}+1, p_{3}=4 q_{3}+2 .
$$

Since $q_{3}$ is odd, by putting $q_{3}=2 k+1$ for $k \in \mathbb{Z}_{\geq 0}$, the representing weight system is given by $(6.22)$.

Case $p_{1}=3$. Then $h e_{W}(h)=r\left\{2 p_{3}-3 q_{3}\right\}-3=h+r\left(p_{3}-2 q_{3}-1\right) 3 / 2+(r / 2-$ 1) $\left(p_{3}+3\right)$. Clearly the second and the third terms are non negative for $r \geq 2$. The last term is positive if $r>2$. Hence $r$ is either 2 or 1 . If $r=2$, then $e(h)=1$ implies $p_{3}=2 q_{3}+1$. On the other hand, $r=2$ means $p_{2}-1=2 q_{3}$. These imply $p_{3}=2 q_{3}+1=p_{2}$, which is impossible. Thus $r=1$ and $h e_{W}(h)=2 p_{3}-3 q_{3}-3$. Thus $e_{W}(h)=1$ implies $p_{3}=3\left(q_{3}+1\right)$ and $p_{3}=q_{3}+1$.

$$
p_{1}=3, p_{2}=q_{3}+1, p_{3}=3\left(q_{3}+1\right)
$$

where $q_{3}$ should obey conditions $\left(p_{1}, p_{2}\right)=\left(p_{3}, q_{3}\right)=1$. The conditions on $q_{3}$ means $q_{3} \not \equiv 0,-1 \bmod 3$. By putting $q_{3}=3 k+1$ for $k \in \mathbb{Z}_{\geq 0}$,

$$
\underline{p}=(3,3 k+2,3(3 k+2))
$$

the representing weight system is given by $(6.23)$.

Case $p_{1}=4$. $h e_{W}(h)=r\left\{3 p_{3}-4 q_{3}\right\}-4=h+r\left(p_{3}-2 q_{3}\right) 2+(r-1) p_{3}-4$. The second term is always positive. Since $p_{1} \neq p_{3}$ and $p_{1}=4$, one has $p_{3}>4$ so that the sum of the last two terms is positive when $r \geq 2$. Therefore, if $e_{W}(h)=1$, then $r=1$ and $p_{3}=2 q_{3}+2$.

$$
p_{1}=4, p_{2}=q_{3}+1, p_{3}=2 q_{3}+2
$$

where $q_{3}$ obeys conditions $\left(p_{1}, p_{2}\right)=2$ and $\left(p_{3}, q_{3}\right)=1$. The conditions on $q_{3}$ mean $q_{3} \not \equiv 0,-1,-2(4)$ and $q_{3} \not \equiv 0 \bmod 2$. By putting $q_{3}=4 k+1$ for $k \in \mathbb{Z}_{>0}$, the representing weight system is given by $(6.24)$.

These complete the proof of Theorem 6.3.

COROLlaRY 6.4. The rank of a simple weight system is determined only by its poset, independent of weight systems representing the poset.

REMARK 6.5. Theorem 6.3 implies that a simple weight system is either nondegenerate dual type or degenerate non-dual type XI, where a weight system is called degenerate if it has exponent 0 (i.e. $a_{0}>0$ ).

The simple weight systems of type XI (6.22-24) seem to give an interesting "series of moderate degeneration" of algebraic varieties. For instance, the initial $(k=0)$ weight system of the series is

$$
\widetilde{E}_{8}:(3,2,1 ; 6)
$$

This is one of three elliptic weight systems corresponding to simply elliptic singularities (see the remarks at the end of $\S 11)$. The next $(k=1)$ weight systems in the series: $(7,2,3 ; 14),(5,3,4 ; 15)$ and $(3,2,5 ; 12)$ are exactly the list of degenerate weight systems having one negative exponent. They correspond to homotopy K3-surfaces with elliptic fibrations studied in [S7, §3]. 
7. Duality among weight systems. In this section, we introduce a concept of duality between two weight systems $W$ and $W^{*}$ (Definition 7.5). The dual $W^{*}$ for a given $W$ may not exist always, but is unique if it exists and then $W$ is the dual of $W^{*}$. The smallest exponent for $W$ and $W^{*}$ coincide (Theorem 7.8).

We give a list of dual pairs of weight systems (Theorem 7.9). As a consequence, we prove that a simple weight system with smallest exponent 1 or -1 has always the dual weight system (Theorem 7.10). This gives an answer to our motivation explained at the introduction (see also $\S 11-12$ ).

Before we define the dual of a weight system, we define the dual characteristic function $\varphi_{W}^{*}(\lambda)$ for any $W$.

Definition 7.1. The dual characteristic function of $W$ is defined by

$$
\varphi_{W}^{*}(\lambda):=\prod_{j \mid h}\left(\lambda^{j}-1\right)^{-e_{W}(h / j)},
$$

whose total degree in $\lambda$ is called the dual rank of $W$ and is given by

$$
\nu_{W}:=-\sum_{j \mid h} j \cdot e_{W}(h / j)
$$

$\in \mathbf{Z}$. The dual rank may not always be positive, since $\varphi_{W}^{*}$ may have poles. We show in the next assertion that $\varphi_{W}^{*}(\lambda)$ for any weight system $W$ have poles at most only at $\lambda=1$ of order $2 a_{0}$.

First, we give a preliminary inequality. Note that the multiplicity of the root $\exp (2 \pi \sqrt{-1} / d)(d \in \mathbb{N} d \mid h)$ in the equation $\varphi_{W}(\lambda)=0$ for a weight system $W$ is $\sum_{d|i| h} e_{W}(i)$. So we have an inequality:

$$
\sum_{d|i| h} e_{W}(i) \geq 0
$$

for any $d \in \mathbb{Z}_{>0}$. The multiplicity of zeros (or, minus of the order of poles) of $\varphi_{W}^{*}(\lambda)$ at $\lambda=\exp (2 \pi \sqrt{-1} / d)$ is given by the sum:

$$
-\sum_{d|j| h} e_{W}(h / j)
$$

\section{ASSERTION 7.2.}

1. The sum (7.4) is non-negative for any $d>1$ with $d \mid h$.

2. The sum (7.4) for $d=1$ taken with the minus sign is equal to $2 a_{0}$, where $a_{0}:=\#\{$ exponents equal to 0$\}$ (cf. (3.4)).

Proof. 1. Put $\xi:=h / d$ and rewrite the sum (7.4): $f_{W}(\xi):=-\sum_{k \mid \xi} e_{W}(k)$. Since $f_{W}(\xi)=f_{W}(\Phi(\xi))$ for $\Phi$ defined in (4.3), we have only to check the non-negativity of $f_{W}(\xi)$ for $\xi \in M(W)$ with $\xi \neq h$. As in the proof of Theorem 5.1, let us denote by $M_{\xi}$ the subposet of $M(W)$ consisting of elements low or equal than $\xi$ so that the summation index $k$ in $f_{W}(\xi)$ runs over the set $M_{\xi}$. We proceed the calculation according to $n(\xi)=0,1$ or 2 .

Case $n(\xi)=0$. Clearly $M_{\xi}=1, f_{W}(\xi)=-e_{W}(1)=-(-1)=1>0$.

Case $n(\xi)=1 . M_{\xi}=$ (1) , so $f_{W}(\xi)=-e_{W}(1)-e_{W}(\xi)=1-1=0$ (Theorem 5.12 ). 
Case $n(\xi)=2$. As in the proof of Theorem 5.1, we consider 3 cases. In any case, recall the fact $-e_{W}(\xi) \geq 1$ (Theorem 5.11 ).

i)<smiles>C=Cc1ccccc1C#CCCC</smiles>

Then $f_{W}(\xi)=-(-1)-(1)-(1)-e_{W}(\xi)=-e_{W}(\xi)-1 \geq 0$.

ii) $M_{\xi}=1$. Then $f_{W}(\xi)=-(-1)-1-e_{W}(\xi) \geq 1$.

iii) $M_{\xi}=\left(1\right.$ - $\xi$. Then $f_{W}(\xi)=-(-1)-e_{W}(\xi)=-e_{W}(\xi)+1 \geq 2$.

2. The left hand side of (7.4) for $d=1$ is equal to the minus of the multiplicity of the root $\lambda=1$ in $\varphi_{W}(\lambda)=0$. Use the formula (3.4).

The weight system $W$ is called non-degenerate if the genus is zero: i.e. $a_{0}=0$. So, the dual characterisitic function $\varphi_{W}^{*}(\lambda)$ is a polynomial, if and only if $W$ is nondegenerate. In general, $(\lambda-1)^{2 a_{0}} \varphi_{W}^{*}(\lambda)$ is a cyclotomic polynomial of degree $\nu_{W}+2 a_{0}$ without a root $\lambda=1$. Let us introduce dual exponents as follows.

Definition 7.3. The system of integers $m_{i}^{*}$ for $1 \leq i \leq \nu_{W}+2 a_{0}$ are called the dual exponents, if $0<m_{i}^{*}<h$ for $1 \leq i \leq \nu_{W}+2 a_{0}$ and

$$
\left(\prod_{i=1}^{\nu+2 a_{0}}\left(\lambda-\exp \left(2 \pi \sqrt{-1} m_{i}^{*} / h\right)\right)\right)=(\lambda-1)^{2 a_{0}} \varphi_{W}^{*}(\lambda) .
$$

REMARK 7.4. Since $\nu_{W}+2 a_{0}$ is not less than the Euler number of $h$, it is positive. But $\nu_{W}\left(=\operatorname{deg}\left(\varphi^{*}\right)\right)$ may be negative (see the $\S 8$ for a formula for $\left.\nu_{W}\right)$. Nevertheless, one has the boundedness of $\nu_{W}$ from below.

$$
\mu_{W}+h \cdot \nu_{W} \geq 0 .
$$

Proof. The LHS of (7.5) is $\sum_{i \in M(W)}\left(i-h^{2} / i\right) e_{W}(i)$. We decompose the sum according $n(i)=0,1,2$ or 3 . The terms for $n(i)=3$ (i.e. $i=h$ ) are cancelled automatically. So,

$$
=-1+h^{2}+\sum_{n(i)=1}\left(i-h^{2} / i\right)+\sum_{n(i)=2}\left(h^{2} / i-i\right) \cdot\left|e_{W}(i)\right|
$$

The last term is automatically non-negative. Therefore, if the index set $I:=\{i \in$ $M(W) \mid n(i)=1\}$ for the second term is empty, the sum is non-negative. Assume $I \neq \emptyset$. Then one has

$$
=\left(\sum_{i \in I} i-1\right)+h^{2}\left(1-\sum_{i \in I} 1 / i\right)+\sum_{n(i)=2}\left(h^{2} / i-i\right) \cdot\left|e_{W}(i)\right|
$$

The set $I$ consists at most of three integers, which are non less than 2 and mutually different (cf. (4.4)). So, the factor $1-\sum_{i \in I} 1 / i$ can be negative only when $I=\{2,3,5\}$. Then the explicit formula for $e_{W}$ in Table A. I, shows that $e_{W}(i)=(-1)^{n(i)}$ and $\mu_{W}=\nu_{W}=8$. So, the formula (7.5) is proven.

DEFINITION 7.5. Let $W$ and $W^{*}$ be weight systems and let $\varphi_{W}$ and $\varphi_{W^{*}}$ be their characteristic polynomials, respectively. We say $W$ is dual to $W^{*}$, if one of the following three conditions is satisfied. 
i) $\varphi_{W} \neq\left(\varphi_{W}\right)^{*}$ and $\varphi_{W^{*}}=\left(\varphi_{W}\right)^{*}\left(\Leftrightarrow\left(\varphi_{W^{*}}\right)^{*}=\varphi_{W}\right)$.

ii) $\varphi_{W}=\left(\varphi_{W}\right)^{*}$ and $W=W^{*}$ except for the next iii).

iii) $\varphi_{W}=\left(\varphi_{W}\right)^{*}$ and $W$ and $W^{*}$ are of the form (6.15) where the order of the parameters $k, l$ and $m$ is reversed in $W$ and $W^{*}$.

By definition, $W$ is dual to $W^{*}$ if and only if $W^{*}$ is dual to $W$. A weight system of the case ii) is called self-dual. The case iii) is introduced from a comparison with a duality in mathematical physics (see [T]). The definition of the duality can be reformulated in terms of cyclotomic exponents as follows. The verification of the equivalence is left to the reader.

Lemma 7.6. A weight system $W$ is dual to $W^{*}$, if and only if the following conditions are satisfied.

i) the Coxeter numbers for $W$ and $W^{*}$ coincide. Put $h:=h_{W}=h_{W^{*}}$.

ii) the involution $\iota$ defined by

$$
\iota(d):=h / d \quad \text { for } \quad d \in \operatorname{Div}(h)
$$

induces an anti-isomorphisms of the posets: $M(W) \simeq M\left(W^{*}\right)$.

iii) for any $\xi \in M(W)$, one has

$$
e_{W}(\xi)+e_{W^{*}}(\iota(\xi))=0
$$

iv) If $M(W)=M\left(W^{*}\right)$, then either $W=W^{*}$, or $W$ and $W^{*}$ are of the form (6.15) with reversed order of parameters $k, l$ and $m$.

Followings are immediate consequences of the definition.

ASSERTION 7.7. i) -iv).

1. Suppose that there is a weight system dual to $W$. Then $W$ satisfies the following

i) $W$ is non-degenerate: i.e. $a_{0}=\#\{0-$ exponents $\}=0$.

ii) $W$ is simple: $\operatorname{mult}(W)=e_{W}(h)=1$.

iii) the poset $M(W)$ is of dual type.

iv) the poset $\iota(M(W))$ is represented by a simple weight system.

2. Conversely, suppose $W$ is simple and $\iota(M(W))$ is represented by a simple weight system, then there is a weight system dual to $W$.

Proof. Assume an existence of a weight system dual to $W$.

i) Since $\varphi_{W}^{*}$ is a polynomial, one has $a_{0} \leq 0$ and hence $a_{0}=0$.

ii) Using (7.7) and Theorem $5.12, e_{W}(h)=-e_{W^{*}}(\iota(h))=-e_{W^{*}}(1)=1$.

iii) Due to the Theorem 6.2 and above ii), $e_{W}(\xi)=(-1)^{n(\xi)}$. Then $\sum_{\xi \in M(W)}(-1)^{n(\xi)}$

$=\sum_{\xi \in M(W)} e_{W}(\xi)=2 a_{0}=0$ (above $\left.\mathrm{i}\right)$ ). This is the definition of the dual type poset (recall (5.7)).

iv) Obviously, $\iota(M(W))$ is represented by a weight system $W^{*}$, which is dual to $W$. Apply above ii) to $W^{*}$ so that it is also simple.

Conversely, if $W^{*}$ is a simple weight system representing $\iota(M(W)$ ) (in case $\iota(M(W))=M(W)$, choose $W^{*}$ carefully in accordance with ii) and iii) of Definition 7.5). Then the simplicity of $W^{*}$ and $W$ together with the formula (6.1) implies the condition (7.7) and $W^{*}$ is dual to $W$.

Combining Assertion 7.7 2 with Theorem 6.3 (which gives conditions for an existence of a simple weight system representing $\iota(M(W)))$, one obtains conditions for a weight system to have a dual. This has two important consequences: i) uniqueness of the dual $W^{*}$, and ii) coincidence of the smallest exponents for $W$ and $W^{*}$, as formulated in the next theorem. 
THEOREM 7.8.

1. The dual weight system of a given weight system $W$ (if it exists) is uniquely determined by $W$. We denote it by $W^{*}$.

2. If $W$ is simple of types either I or III then $W$ is self-dual. If $W$ is simple of type $\mathrm{V}$, then $W$ is either of type $A_{h-1}$ or of the form (6.15).

3. The smallest exponents for $W$ and $W^{*}$ coincide with each other.

Proof. 1. Let $W$ be dual to $W^{*}$. If $\iota(M(W))=M(W)$, then $W$ is either self-dual or of the form (6.15) so the dual $W^{*}$ is uniquely defined. If $\iota(M(W)) \neq M(W)$, then we have to show the uniqueness of simple weight system representing $\iota(M(W))$. Since $M(W)$ is of dual type (Assertion $7.7 \mathrm{iii})), \iota(M(W))$ is also of the same type and hence one of the types $\mathrm{I} \sim \mathrm{V}$. We know already by Theorem $6.3(\S 6)$ that a poset of type II or IV can be always represented by a unique simple weight system. Therefore, it is sufficient to show that if $M(W)$ is of type I, III or $\mathrm{V}$, then one has $\iota(M(W))=M(W)$. Actually, this is proven in the next 2.

2. Let $M(\underline{p})$ be simple and of type either I, III or V. We show $\iota(M(\underline{p}))=M(\underline{p})$ for each types separately.

I. Recall Table A I and Theorem 6.3 I. The simplicity of $M(\underline{p})$ implies $p_{123}=$ $p_{1} p_{2} p_{3}$ and $p_{i j}=p_{i} p_{j}$ for $i \neq j$. Then, $\iota\left(p_{i}\right)\left(=p_{123} / p_{i}\right)$ is equal to $p_{j k}$ for $\{i, j, k\}=$ $\{1,2,3\}$. This implies $\iota(M(p))=M(\underline{p})$.

III. Recall Table A III and Theorem 6.3 III. Since $\left(p_{1}, p_{2}\right)=1, p_{2}=p_{3}$ and $h=p_{1} p_{2}$, one has $\iota\left(p_{1}\right)=p_{2}$. This implies $\iota(M(p))=M(p)$.

V. Since $p_{1}=p_{2}=p_{3}=h$, clearly, $\iota(M(p))=M(p)$.

3. If $W$ is self-dual, then $\varepsilon(W)=\varepsilon\left(W^{*}\right)$ and we finished the proof. If $W$ is not self-dual, then because of $2, M(W)$ is either of the form (6.15) or of type II or IV. The smallest exponent (1.5) for the case $(6.15)$ is given by $1-(k-1)(l-1)(m-1)$, which is invariant under the change of the order of $k, l$ and $m$. We prove the equality $\varepsilon(W)=\varepsilon\left(W^{*}\right)$ separately for the types II and IV in the following Theorem 7.9.

In the next Theorem 7.9, we list all dual pairs of weight systems and their smallest exponents. The result is described in terms of the poset $M(\underline{p})$ generated by three integers $\underline{p}=\left(p_{1}, p_{2}, p_{3}\right)$ with $p_{i} \geq 2$ (cf. $\left.\S 4\right)$, where $h=\operatorname{lcm}\left(p_{1}, p_{2}, p_{3}\right)$, and $\iota$ is the involution $\iota(\xi):=h / \xi$.

THEOREM 7.9. Let $M(\underline{p})$ be a simple poset generated by $\underline{p}=\left(p_{1}, p_{2}, p_{3}\right)$ with $p_{i} \geq 2$ (cf. Theorem 6.3). For each type of $M(\underline{p})$, we give a list of

i) the conditions for $\iota(M(p))$ to be simple,

ii) simple weight systems $\bar{W}$ and $W^{*}$ representing $M(\underline{p})$ and $\iota(M(\underline{p}))$,

iii) the smallest exponents $\varepsilon:=\varepsilon_{W}=\varepsilon_{W^{*}}$ for $W$ and $W^{*}$,

iv) the condition for $W$ to be self-dual.

I. Let $M(p)$ be simple of type I (cf. (6.2)).

i) $\iota(M(\underline{p}))=M(\underline{p})$. It is automatically simple.

ii) The weight system representing $M(\underline{p})=\iota M(\underline{p})$ is uniquely given by

$$
W=W^{*}:=\left(p_{1} p_{2}, p_{2} p_{3}, p_{3} p_{1} ; p_{1} p_{2} p_{3}\right) .
$$

iii) $\quad \varepsilon_{W}=h \cdot\left(1 / p_{1}+1 / p_{2}+1 / p_{3}-1\right)$,

iv) $W$ is automatically self-dual.

II. Let $M(p)$ be simple of type II (cf. (6.5)).

i) $\iota(M(\underline{p})) \stackrel{=}{=} M\left(p_{1}, p_{3} / p_{2}, p_{3}\right)$. It is simple if and only if

$$
\left(p_{3} / p_{2}-1, p_{3}\right)=1
$$


ii) The weight system representing $M(\underline{p})$ and $\iota(M(\underline{p}))$ are uniquely given by

$$
\begin{aligned}
& W=\left(p_{3}, p_{1} p_{3} / p_{2},\left(p_{2}-1\right) p_{1} ; p_{1} p_{3}\right) \\
& W^{*}=\left(p_{3}, p_{1} p_{2},\left(p_{3} / p_{2}-1\right) p_{1} ; p_{1} p_{3}\right)
\end{aligned}
$$

iii)

$$
\varepsilon_{W}=p_{3}-p_{1}\left(p_{2}-1\right)\left(p_{3} / p_{2}-1\right) .
$$

iv) The weight system $W$ is self-dual if and only if

$$
p_{2}^{2}=p_{3}
$$

III. Let $M(\underline{p})$ be simple of type III (cf. (6.8)).

i) $\iota(M(\underline{p}))=M(\underline{p})$. It is automatically simple.

ii) The weight systems representing $M(\underline{p})=\iota(M(\underline{p}))$ are given by $(6.9)$ :

$$
W=W^{*}=\left(p_{2}, p_{1} q_{2}, p_{1} q_{3} ; p_{1} p_{2}\right) .
$$

iii) $\quad \varepsilon_{W}=-\left(p_{1}-1\right)\left(p_{2}-q_{2}-q_{3}\right)+q_{2}+q_{3}$.

iv) Any simple weight system representing $M(\underline{p})$ is self-dual.

IV. Let $M(p)$ be simple of type IV (cf. (6.11)).

i) $\iota(M(p))=M\left(p_{3} / p_{2}, p_{3} / p_{1}, p_{3}\right)$. It is simple if and only if

$$
\left(p_{3} / p_{2}-1, p_{3} / p_{1}\right)=\left(p_{3} / p_{1}-p_{3} / p_{2}+1, p_{3}\right)=1 .
$$

ii) The weight systems representing $M(\underline{p})$ and $\iota(M(\underline{p}))$ are uniquely given by

$$
\begin{aligned}
& W=\left(p_{3} / p_{1},\left(p_{1}-1\right) p_{3} / p_{2}, p_{2}-p_{1}+1 ; p_{3}\right), \\
& \left.W^{*}=\left(p_{2},\left(p_{3} / p_{2}-1\right) p_{1}, p_{3} / p_{1}-p_{3} / p_{2}+1 ; p_{3}\right)\right) .
\end{aligned}
$$

iii)

$$
\begin{aligned}
\varepsilon_{W} & =p_{3} / p_{1}-\left(p_{3} / p_{2}-1\right)\left(p_{2}-p_{1}+1\right) \\
& =p_{3}-p_{2} p_{3} / p_{1}-\left(p_{3} / p_{1}-p_{3} / p_{2}+1\right)\left(p_{2}-p_{1}+1\right) .
\end{aligned}
$$

iv) The weight system $W$ is self-dual if and only if

$$
p_{1} p_{2}=p_{3} .
$$

V. Let $M(\underline{p})$ be simple of type V (cf. (6.14)).

i) $\iota(M(\underline{p}))=M(\underline{p})$. It is automatically simple.

ii) The weight systems representing $M(\underline{p})=\iota(M(\underline{p}))$ are either of type $A_{h-1}$ or given by (6.15):

$$
\begin{aligned}
& W=(l m-m+1, m k-k+1, k l-l+1 ; k l m+1), \\
& W^{*}=(l k-k+1, m l-l+1, k m-m+1 ; k l m+1) .
\end{aligned}
$$

iii) $\quad \varepsilon_{W}=1-(k-1)(l-1)(m-1)$.

iv) A simple weight system representing $M(\underline{p})$ is self-dual if either it is of type $A_{h-1}$ or of the form (6.15) and $k=l, l=m$ or $m=k$. 
VI-XIV. If $M(\underline{p})$ is one of types VI-XIV, then it is not of dual type and $\iota(M(p))$ cannot be represented by a weight system.

Proof. The calculations of i), ii) and iv) are easy exercise by a use of data and results of Theorem $6.3 \S 6$. The only thing one need to show is the coincidence of the smallest exponents of $W$ and $W^{*}$ for types II and IV. Recalling the formula $\varepsilon=a+b+c-h$ for the smallest exponent, we show the coincidence of the sum $a+b+c$ of the weights for each cases.

I. Recall Theorem 6.3 I and Theorem 7.8 2.

II. Recall Table A II and Theorem 6.3 II. Since $p_{2} \neq p_{3},\left(p_{1}, p_{3}\right)=1$ and $h=p_{1} p_{3}$, one has $\iota\left(p_{1}\right)=p_{3}, \iota\left(p_{2}\right)=p_{1}\left(p_{3} / p_{2}\right), \iota\left(p_{1} p_{2}\right)=p_{3} / p_{2} \geq 2$ and $\iota\left(p_{3}\right)=p_{1}$. Put $p_{1}^{\prime}:=p_{1}, p_{2}^{\prime}:=p_{3} / p_{2}$ and $p_{3}^{\prime}:=p_{3}$. Clearly $\left(p_{1}^{\prime}, p_{3}^{\prime}\right)=1, p_{2}^{\prime} \neq p_{3}^{\prime}$ and $p_{1}^{\prime} \vee p_{2}^{\prime}=p_{1}^{\prime} p_{2}^{\prime}=$ $p_{1}\left(p_{3} / p_{2}\right)$. So, $\iota(M(\underline{p}))=M\left(\underline{p}^{\prime}\right)$. The simplicity conditions for $M\left(\underline{p}^{\prime}\right)$ are $p_{3}^{\prime} / p_{1}^{\prime}(=$ $\left.p_{3} / p_{1}\right) \neq 1,\left(p_{1}^{\prime}, p_{3}^{\prime}\right)\left(\equiv\left(p_{1}, p_{3}\right)\right)=1$ and $\left(p_{2}^{\prime}-1, p_{3}\right)=\left(p_{3} / p_{2}-1, p_{3}\right)=1$. The simple weight system $W^{\prime}$ representing $M\left(\underline{p}^{\prime}\right)$ is uniquely given by $W^{*}=\left(p_{3}, p_{1} p_{2},\left(p_{3} / p_{2}-\right.\right.$ 1) $p_{1} ; p_{1} p_{3}$ ) (cf. (6.7)). By a use of these expressions of the weights, we see that the sum $a+b+c=p_{3}+p_{1} p_{3} / p_{2}+\left(p_{2}-1\right) p_{1}$ and $a^{\prime}+b^{\prime}+c^{\prime}=p_{3}+p_{1} p_{2}+\left(p_{3} / p_{2}-1\right) p_{1}$ coincide. This proves $\varepsilon_{W}=\varepsilon_{W^{*}}$.

III. Recall Theorem 6.3 III and Theorem 7.82 .

IV. Recall Table A, IV and Theorem 6.3 IV. Since $1 \neq p_{1} \neq p_{2} \neq p_{3}=h$, one has $1 \neq p_{3} / p_{2} \neq p_{3} / p_{1} \neq p_{3}$ so that $\iota(M(W))$ is given by $M\left(p^{\prime}\right)$ for $p_{1}^{\prime}=h / p_{2}, p_{2}^{\prime}=h / p_{1}$ and $p_{3}^{\prime}=p_{3}=h$. Then the simplicity conditions for $M\left(\underline{p}^{\prime}\right)$ is: $\left(p_{1}^{\prime}-1, p_{2}^{\prime}\right)=\left(p_{3} / p_{2}-\right.$ $\left.1, p_{3} / p_{1}\right)=1$ and $\left(p_{2}^{\prime}-p_{1}^{\prime}+1, p_{3}^{\prime}\right)=\left(p_{3} / p_{1}-p_{3} / p_{2}+1, p_{3}\right)=1$. The weight system representing $M\left(p^{\prime}\right)$ is uniquely given by $W^{*}=\left(p_{2},\left(h / p_{2}-1\right) p_{1}, h / p_{1}-h / p_{1}+1 ; h\right)$ (cf. (6.13)). By a use of these expression of weights, one see that the sum $a+b+c=$ $h / p_{1}+\left(p_{1}-1\right) h / p_{2}+p_{2}-p_{1}+1$ and $a^{\prime}+b^{\prime}+c^{\prime}=p_{2}+\left(h / p_{2}-1\right) p_{1}+h / p_{1}-h / p_{2}+1$ coincide. This proves $\varepsilon_{W}=\varepsilon_{W^{*}}$.

V. Recall Theorem $6.3 \mathrm{~V}$ and Theorem 7.82 .

As an application of Theorems 7.8 and 7.9, we obtain the following existence of the duality among weight systems with the smallest exponent equal to 1 or -1 , respectively. This result is a starting point of the present work as explained in introduction. In $\S 12$ and 13, we shall study these cases more closely.

THEOREM 7.10. Let $W$ be a primitive weight system.

1. If all exponents are positive, then $W$ is self-dual.

2. If $W$ is simple and non-degenerate with $\varepsilon_{W}=-1$, then it is dual to a weight system with $\varepsilon_{W}=-1$.

Proof. 1. Suppose that all exponents for $W$ are positive. Due to the positivity mult $(W)>0$ (cf. (5.6) and Theorem 5.11 ) and non-existence of exponent -1 , there exists exponent equal to 1 , which should be the smallest. Then due to the simplicity of the smallest exponent (cf. (1.5)), $W$ is simple: mult $(W)=1$. Therefore, $M(W)$ is either of types I $\sim \mathrm{V}$ or of type XI (Theorem 6.22 ). But $W$ cannot be of type XI, for the smallest exponent for type XI is non-positive (Theorem 6.3 XI). The weight system $W$ is already self-dual, if $M(W)$ is of type either I, III or V (Theorem 7.82 ). Thus we have only to show that $W$ satisfies the self-duality conditions in Theorem 7.9 , when $M(W)$ is of type II or IV.

II. Recall descriptions (6.5), (6.6) of a simple weight system $W$ of type II. Note that the (7.10) gives the smallest exponent for $W$, even $W^{*}$ may not exist. Put $u:=p_{3} / p_{2}-1 \geq 1$ and $v:=p_{2}-1 \geq 1$ so that (7.10) yields $p_{1} u v=(u+1)(v+1)-\varepsilon$. Let us show $p_{1} \leq 3$. Otherwise $p_{1} \geq 4$ and so $p_{1} u v \geq 2 u \cdot 2 v \geq(u+1)(v+1)>$ $(u+1)(v+1)-1$, a contradiction! If $p_{1}=3$ then $(2 u-1)(2 v-1)=1$, whose only 
positive integral solution is $u=v=1$. If $p_{1}=2$ then $(u-1)(v-1)=1$, whose only positive integral solution is $u=v=2$. In both cases, one has $u=v$ satisfying the condition (7.11) of the self-duality.

IV. Recall descriptions (6.11), (6.12) of a simple weight system $W$ of type IV. Put $u:=p_{3} / p_{2}-1 \geq 1$ and $v:=p_{2} / p_{1}-1 \geq 1$ so that (7.14) yields $\varepsilon=(u+1)(v+$ $1)-u\left(p_{1} v+1\right)$. This implies $\left(\left(p_{1}-1\right) u-1\right) v=0$. Since $u \geq 1$ and $v \geq 1$, this is possible only when $p_{1}=2$ and $u=1$. So $p_{3} / p_{2}=2$ and hence $W$ satisfies the condition $p_{1}=p_{3} / p_{2}$ (7.15) of the self-duality.

2. Let $W$ be a simple weight system with the smallest exponent equal to -1 . The simplicity implies that $W$ is either of types I $\sim \mathrm{V}$ or type XI (Theorem 6.2). But $W$ cannot be of type XI, since the smallest exponents for the type XI is a multiple of 2 , 3 or 4 (Theorem $6.3 \mathrm{XI}$ ). If $M(W)$ is of type I, III or $\mathrm{V}$, then $W$ is already self-dual (Theorem 7.82 ). Therefore we have only to show that the conditions for the existing of $W^{*}$ in Theorem 7.9 are satisfied, if $W$ is of type II or IV. It is already shown that the smallest exponent of $W^{*}$ is equal to that of $W$ (Theorem 7.9).

II. Recall again in the descriptions (6.5), (6.6) and (7.10). Since $p_{1}\left(p_{3} / p_{2}-1\right)\left(p_{2}-\right.$ $1)=p_{3}-\varepsilon$ so that $p_{3} \equiv \varepsilon \bmod p_{3} / p_{2}-1$. So if $\varepsilon= \pm 1$ then $\left(p_{3}, p_{3} / p_{2}-1\right)=1$, which is the condition (7.7) for the existence of simple dual weight system $W^{*}$.

IV. Recall again the descriptions $(6.11),(6.12)$ and (7.14). Since $\left(p_{3} / p_{2}-1\right)\left(p_{2}-\right.$ $\left.p_{1}+1\right)=p_{3} / p_{1}-\varepsilon$. Hence $p_{3} / p_{1} \equiv \varepsilon \bmod p_{3} / p_{2}-1$. So if $\varepsilon= \pm 1$, then $\left(p_{3} / p_{2}-\right.$ $\left.1, p_{3} / p_{1}\right)=1$. Further, recall $-\left(p_{3} / p_{1}-p_{3} / p_{2}+1\right)\left(p_{2}-p_{1}+1\right)=p_{3}-p_{2} p_{3} / p_{1}-\varepsilon=$ $-\varepsilon+p_{3}\left(1-p_{2} / p_{1}\right)$. Hence $\left(p_{3} / p_{1}-p_{3} / p_{2}+1\right)\left(p_{2}-p_{1}+1\right) \equiv \varepsilon \bmod p_{3}$. This implies that $p_{3} / p_{1}-p_{3} / p_{2}+1$ is a unit in the ring $\mathbb{Z} / \mathbb{Z} p_{3}$. Thus $\left(p_{3} / p_{1}-p_{3} / p_{2}+1, p_{3}\right)=1$. Therefore the conditions (7.12) for $\iota(M(W))$ to be representable by a simple weight system $W^{*}$ are satisfied.

EXAMPLE 7.11.

1. Let $p$ be any integer $\geq 2$ prime to 7 . Put,

$$
W_{1}:=(7, p, 3 p ; 7 p) \text { and } W_{2}:=(7, p, 2 p ; 7 p) \text {. }
$$

Then they have the same poset: $M\left(W_{1}\right)=M\left(W_{2}\right)=$ $e_{W_{1}}(h)=-e_{W_{1}}(7)=1$ and $e_{W_{2}}(h)=-e_{W_{2}}(7)=2$ (use Table A III). So, $W_{1}$ is simple and self-dual but $W_{2}$ is non-degenerate but not simple.

2. Many examples of simple non-degenerate weight system, which are not dual to any other weight system, are given in the Appendix.

8. Dual rank $\nu_{W}$ and genus $a_{0}$. In this section we give formulae of the dual rank $\nu_{W}$ and the genus $a_{0}$ in terms of, so called, refined coordinates of $W$, (see (8.4) and (8.5)). As a consequence, we get a criterion for $\nu_{W}$ to be positive or negative.

The refined coordinates for $W$ is a system of integers $\bar{p}_{1}, \bar{p}_{2}, \bar{p}_{3}, u, v, w, m$ and $q_{1}, q_{2}, q_{3}$ describing the weight system $W$ (see (8.1)-(8.3)). It is cumbersome to use such coordinates, but, the results in this and next sections, in particular, the integrality of the discriminants (Theorem 9.2) are proven only by a use of them. The results are closely related to our next goal on eta-products in $\$ 14$, but will not be used immediately. Therefore, some readers are suggested to skip this and the next sections.

Let $W=(a, b, c ; h)$ be a weight system. Let $p_{1}, p_{2}$ and $p_{3}$ be the generators of the poset $M(W)$ defined in (4.4)-(4.7). We consider the following refinement of $p_{i}$ :

$$
p_{1}=\bar{p}_{1} v w m, p_{2}=\bar{p}_{2} w u m \text { and } p_{3}=\bar{p}_{3} u v m
$$


where $\bar{p}_{1}, \bar{p}_{2}, \bar{p}_{3}, u, v, w$ and $m$ are positive integers determined by the rule:
i) $\bar{p}_{1}, \bar{p}_{2}$ and $\bar{p}_{3}$ are mutually prime to each other,
ii) $u, v$ and $w$ are mutually prime to each other,
iii) $\left(\bar{p}_{1}, u\right)=\left(\bar{p}_{2}, v\right)=\left(\bar{p}_{3}, w\right)=1$,
iv) $\left(q_{1}, \bar{p}_{1} v w m\right)=\left(q_{2}, \bar{p}_{2} w u m\right)=\left(q_{3}, \bar{p}_{3} u v m\right)=1$.

We call the system of integers $\bar{p}_{1}, \bar{p}_{2}, \bar{p}_{3}, u, v, w, m$ and $q_{1}, q_{2}, q_{3}$ the refined coordinates for the weight system $W$. Refined coordinates exist uniquely (put $m:=\operatorname{gcd}\left(p_{1}, p_{2}, p_{3}\right)$, $u:=\operatorname{gcd}\left(p_{2} / m, p_{3} / m\right), v:=\operatorname{gcd}\left(p_{3} / m, p_{1} / m\right)$ and $\left.w:=\operatorname{gcd}\left(p_{1} / m, p_{2} / m\right)\right)$. The word "coordinate" is justified, since the weight system is recovered from the refined coordinates as

$$
h=\bar{p}_{1} \bar{p}_{2} \bar{p}_{3} u v w m, a=q_{1} \bar{p}_{2} \bar{p}_{3} u, b=q_{2} \bar{p}_{3} \bar{p}_{1} v, c=q_{3} \bar{p}_{1} \bar{p}_{2} w,
$$

or, equivalently

$$
W=\left(q_{1} \bar{p}_{2} \bar{p}_{3} u, q_{2} \bar{p}_{3} \bar{p}_{1} v, q_{3} \bar{p}_{1} \bar{p}_{2} w ; \bar{p}_{1} \bar{p}_{2} \bar{p}_{3} u v w m\right) .
$$

The weight system $W(8.3)$ is regular, if and only if the coordinates satisfy

v) $q_{1}, q_{2}$ and $q_{3}$ are mutually prime to each other,

vi) either $q_{1}=1, \bar{p}_{2} u=1 \& q_{1} \mid\left(w m-q_{2}\right)$, or $\bar{p}_{3} u=1 \& q_{1} \mid\left(v m-q_{3}\right)$,

vii) either $q_{2}=1, \bar{p}_{3} v=1 \& q_{2} \mid\left(u m-q_{3}\right)$, or $\bar{p}_{1} v=1 \& q_{2} \mid\left(w m-q_{1}\right)$,

viii) either $q_{3}=1, \bar{p}_{1} w=1 \& q_{3} \mid\left(v m-q_{1}\right)$, or $\bar{p}_{2} w=1 \& q_{3} \mid\left(u m-q_{2}\right)$.

For a proof, see remarks at (1.1) and (4.8).

THEOREM 8.1. Let the notation be as above. Then one has

$$
\begin{aligned}
\nu_{W}= & \bar{p}_{1} \bar{p}_{2} \bar{p}_{3} u v w m-\left(\bar{p}_{2} \bar{p}_{3} u / q_{1}+\bar{p}_{1} \bar{p}_{3} v / q_{2}+\bar{p}_{1} \bar{p}_{2} w / q_{3}\right) \\
& +\left(\bar{p}_{1} u / q_{2} q_{3}+\bar{p}_{2} v / q_{1} q_{3}+\bar{p}_{3} w / q_{1} q_{2}\right) m-u v w m^{2} / q_{1} q_{2} q_{3} . \\
2 a_{0}= & u v w m^{2} / q_{1} q_{2} q_{3}-\left(u / q_{2} q_{3}+v / q_{1} q_{3}+w / q_{1} q_{2}\right) m \\
& +1 / q_{1}+1 / q_{2}+1 / q_{3}-1 .
\end{aligned}
$$

Proof. The proof depends on the type of $W$ using the next Table G. One has to verify that the formulae c) and d) in the table are the specializations of the formulae (8.4) and (8.5) by applying the conditions a) in the table. Details of the calculations are left to the reader.

Table G.

For each type of weight system $W$, following data are exhibited.

a) Numerical conditions on the refined coordinates for $W$ to be of the type, and explicit description of $W$.

b) Explicit formula of the cyclotomic exponents obtained by rewriting the exponents given in the Table $\mathrm{A}$,

c) Explicit formula of the genus obtained by rewriting (3.4),

d) Explicit formula of the dual rank obtained by rewriting (7.2)

\section{Type I}

a) $\bar{p}_{1} \neq 1, \bar{p}_{2} \neq 1, \bar{p}_{3} \neq 1, q_{1}=q_{2}=q_{3}=1$.

$W=\left(\bar{p}_{2} \bar{p}_{3} u, \bar{p}_{3} \bar{p}_{1} v, \bar{p}_{1} \bar{p}_{2} w ; \bar{p}_{1} \bar{p}_{2} \bar{p}_{3} u v w m\right)$. 
b) $e_{W}(h)=u v w m^{2}, e_{W}\left(p_{12}\right)=-w m, e_{W}\left(p_{23}\right)=-u m, e_{W}\left(p_{31}\right)=-v m$.

c) $2 a_{0}=u v w m^{2}-(u+v+w) m+2$,

d) $\nu_{W}=-u v w m^{2}+\left(\bar{p}_{1} \bar{p}_{2} \bar{p}_{3} u v w+\bar{p}_{1} u+\bar{p}_{2} v+\bar{p}_{3} w\right) m-\left(\bar{p}_{2} \bar{p}_{3} u+\bar{p}_{3} \bar{p}_{1} v+\bar{p}_{1} \bar{p}_{2} w\right)$.

Type II.

a) $\bar{p}_{1} \neq 1, \bar{p}_{2}=1, \bar{p}_{3} \neq 1, u \neq 1, w=1, q_{1}=q_{2}=1, q_{3} \mid u m-1$.

$W=\left(\bar{p}_{3} u, \bar{p}_{3} \bar{p}_{1} v, q_{3} \bar{p}_{1} ; \bar{p}_{1} \bar{p}_{3} u v m\right)$.

b) $e_{W}(h)=(u m-1) v m / q_{3}, e_{W}\left(p_{12}\right)=-m, e_{W}\left(p_{3}\right)=(u m-1) / q_{3}$,

c) $2 a_{0}=(u m-1)(v m-1) / q_{3}-m+1$,

d) $\nu_{W}=-u v m^{2} / q_{3}+\left(\bar{p}_{1} \bar{p}_{3} u v+\bar{p}_{1} u / q_{3}+v / q_{3}+\bar{p}_{3}\right) m-\left(\bar{p}_{3} u+\bar{p}_{3} \bar{p}_{1} v+\bar{p}_{1} / q_{3}\right)$.

Type III.

a) $\bar{p}_{1} \neq 1, \bar{p}_{2}=\bar{p}_{3}=1, u \neq 1, v=w=1, q_{1}=1, q_{2}\left|u m-q_{3}, q_{3}\right| u m-q_{2}$,

$W=\left(u, q_{2} \bar{p}_{1}, q_{3} \bar{p}_{1} ; \bar{p}_{1} u m\right)$.

b) $e_{W}(h)=m\left(u m-q_{2}-q_{3}\right) / q_{2} q_{3}, e_{W}\left(p_{2}\right)=-\left(u m-q_{2}-q_{3}\right) / q_{2} q_{3}$,

c) $2 a_{0}=(m-1)\left(u m-q_{2}-q_{3}\right) / q_{2} q_{3}$,

d) $\nu_{W}=-u m^{2} / q_{2} q_{3}+\left(\bar{p}_{1} u+\bar{p}_{1} u / q_{2} q_{3}+1 / q_{3}+1 / q_{2}\right) m-\left(u+\bar{p}_{1} / q_{2}+\bar{p}_{1} / q_{3}\right)$.

Type IV.

a) $\bar{p}_{1}=\bar{p}_{2}=1, \bar{p}_{3} \neq 1, u \neq 1, v=w=1, q_{1}=1, q_{2}\left|m-1, q_{2} q_{3}\right|$,

$W=\left(\bar{p}_{3} u, q_{2} \bar{p}_{3}, q_{3} ; \bar{p}_{3} u m\right)$.

b) $e_{W}(h)=(m-1)\left(m u-q_{2}\right) / q_{2} q_{3}, e_{W}\left(p_{2}\right)=-(m-1) / q_{2}$,

c) $2 a_{0}=(m-1)\left(u m-q_{2}-q_{3}\right) / q_{2} q_{3}$,

d) $\nu_{W}=-(m-1)\left(u m-u \bar{p}_{3} q_{2} q_{3}-q_{2}-\bar{p}_{3} q_{3}\right) / q_{2} q_{3}$,

Type V.

a) $\bar{p}_{1}=\bar{p}_{2}=\bar{p}_{3}=1, u=v=w=1, m \neq 1,\left(m, q_{i}\right)=1$,

$W=\left(q_{1}, q_{2}, q_{3} ; m\right)$.

b) $e_{W}(h)=\left(\left(m-q_{1}\right)\left(m-q_{2}\right)\left(m-q_{3}\right) / q_{1} q_{2} q_{3}+1\right) / m$

$=m^{2} / q_{1} q_{2} q_{3}-\left(1 / q_{1} q_{2}+1 / q_{2} q_{3}+1 / q_{3} q_{1}\right) m+1 / q_{1}+1 / q_{2}+1 / q_{3}$,

c) $2 a_{0}=m^{2} / q_{1} q_{2} q_{3}-\left(1 / q_{1} q_{2}+1 / q_{2} q_{3}+1 / q_{3} q_{1}\right) m+1 / q_{1}+1 / q_{2}+1 / q_{3}-1$,

d) $\nu_{W}=-m^{2} / q_{1} q_{2} q_{3}+\left(1 / q_{1} q_{2}+1 / q_{2} q_{3}+1 / q_{3} q_{1}+1\right) m-1 / q_{1}-1 / q_{2}-1 / q_{3}$,

Type VI.

a) $\bar{p}_{1} \neq 1, \bar{p}_{2}=\bar{p}_{3} \neq 1, u \neq 1, w \neq 1, q_{1}=q_{2}=q_{3}=1$,

$W=\left(\bar{p}_{3} u, \bar{p}_{3} \bar{p}_{1} v, \bar{p}_{1} w ; \bar{p}_{1} \bar{p}_{3} u v w m\right)$.

b) $e_{W}(h)=(u w m-1) v m, e_{W}\left(p_{12}\right)=-w m, e_{W}\left(p_{23}\right)=-u m$,

c) $2 a_{0}=u v w m^{2}-(u+v+w) m+2$.

d) $\nu_{W}=-u v w m^{2}+\left(\bar{p}_{1} \bar{p}_{3} u v w+\bar{p}_{1} u+v+\bar{p}_{3} w\right) m-\left(\bar{p}_{3} u+\bar{p}_{1} \bar{p}_{3} v+\bar{p}_{1} w\right)$.

Type VII.

a) $\bar{p}_{1} \neq 1, \bar{p}_{2}=\bar{p}_{3}=1, u \neq 1, v \neq 1, w \neq 1, q_{1}=q_{2}=q_{3}=1$,

$W=\left(u, \bar{p}_{1} v, \bar{p}_{1} w ; \bar{p}_{1} u v w m\right)$.

b) $e_{W}(h)=m(u v w m-v-w), e_{W}\left(p_{23}\right)=-u m$,

c) $2 a_{0}=u v w m^{2}-(u+v+w) m+2$,

d) $\nu_{W}=-u v w m^{2}+\left(\bar{p}_{1} u v w+\bar{p}_{1} u+v+w\right) m-\left(u+\bar{p}_{1} v+\bar{p}_{1} w\right)$.

Type VIII.

a) $\bar{p}_{1}=\bar{p}_{2}=\bar{p}_{3}=1, u \neq 1, v \neq 1, w \neq 1, q_{1}=q_{2}=q_{3}=1$, 
$W=(u, v, w ; u v w m)$.

b) $e_{W}(h)=u v w m^{2}-(u+v+w) m$,

c) $2 a_{0}=u v w m^{2}-(u+v+w) m+2$,

d) $\nu_{W}=(1-m)(m u v w-u-v-w)$.

Type IX.

a) $\bar{p}_{1} \neq 1, \bar{p}_{2}=\bar{p}_{3}=1, u \neq 1, v \neq 1, w=1, q_{1}=q_{2}=1, q_{3} \mid u m-1$,

$W=\left(u, \bar{p}_{1} v, q_{3} \bar{p}_{1} ; \bar{p}_{1} u v m\right)$.

b) $e_{W}(h)=m\left(u v m / q_{3}-v / q_{3}-1\right), e_{W}\left(p_{3}\right)=-(u m-1) / q_{3}$,

c) $2 a_{0}=u v m^{2} / q_{3}-\left(u / q_{3}+v / q_{3}+1\right) m+1 / q_{3}+1$,

d) $\nu_{W}=-u v m^{2} / q_{3}+\left(\bar{p}_{1} u v+\bar{p}_{1} u / q_{3}+v / q_{3}+1\right) m-\left(u+\bar{p}_{1} v+\bar{p}_{1} / q_{3}\right)$.

Type X.

a) $\bar{p}_{1}=\bar{p}_{2}=1, \bar{p}_{3} \neq 1, u \neq 1, v \neq 1, w=1, q_{1}=q_{2}=1, q_{3} \mid u m-1$ or $v m-1$

$W=\left(\bar{p}_{3} u, \bar{p}_{3} v, q_{3} ; \bar{p}_{3} u v m\right)$.

b) $e_{W}(h)=(u m-1)(v m-1) / q_{3}, e_{W}\left(p_{12}\right)=-m$,

c) $2 a_{0}=u v m^{2} / q_{3}-\left(u / q_{3}+v / q_{3}+1\right) m+1 / q_{3}+1$,

d) $\nu_{W}=-u v m^{2} / q_{3}+\left(\bar{p}_{3} u v+u / q_{3}+v / q_{3}+\bar{p}_{3}\right) m-\left(\bar{p}_{3} u+\bar{p}_{3} v+1 / q_{3}\right)$.

Type XI.

a) $\bar{p}_{1}=\bar{p}_{2}=\bar{p}_{3}=1, u \neq 1, v \neq 1, w=1, q_{1}=q_{2}=1, q_{3} \mid u m-1$ or $v m-1$,

$W=\left(u, v, q_{3} ; \bar{p}_{3} u v m\right)$.

b) $e_{W}(h)=(u m-1)(v m-1) / q_{3}-m$,

c) $2 a_{0}=u v m^{2} / q_{3}-\left(u / q_{3}+v / q_{3}+1\right) m+1 / q_{3}+1$,

d) $\nu_{W}=-u v m^{2} / q_{3}+\left(u v+u / q_{3}+v / q_{3}+1\right) m-\left(u+v+1 / q_{3}\right)$.

Type XII.

a) $\bar{p}_{1}=1, \bar{p}_{2} \neq 1, \bar{p}_{3} \neq 1, v=w=1, m \neq 1, q_{1}=1, q_{2}\left|m-1, q_{3}\right| m-1$,

$W=\left(\bar{p}_{2} \bar{p}_{3} u, q_{2} \bar{p}_{3}, q_{3} \bar{p}_{2} ; \bar{p}_{2} \bar{p}_{3} u m\right)$.

b) $e_{W}(h)=(m-1) u m / q_{2} q_{3}, e_{W}\left(p_{2}\right)=(m-1) / q_{2}, e_{W}\left(p_{3}\right)=(m-1) / q_{3}$.

c) $2 a_{0}=u m^{2} / q_{2} q_{3}-\left(u / q_{2} q_{3}+1 / q_{3}+1 / q_{2}\right) m+1 / q_{2}+1 / q_{3}$,

d) $\nu_{W}=(m-1)\left(\bar{p}_{2} \bar{p}_{3} u+\bar{p}_{3} / q_{2}+\bar{p}_{2} / q_{3}-u / q_{2} q_{3} m\right)$

$=-u m^{2} / q_{2} q_{3}+\left(\bar{p}_{2} \bar{p}_{3} u+u / q_{2} q_{3}+\bar{p}_{2} / q_{3}+\bar{p}_{3} / q_{2}\right) m-\left(\bar{p}_{2} \bar{p}_{3} u+\bar{p}_{3} / q_{2}+\bar{p}_{2} / q_{3}\right)$.

Type XIII.

a) $\bar{p}_{1}=\bar{p}_{2}=\bar{p}_{3}=1, u \neq 1, v=w=1, m \neq 1, q_{1}=1$,

$W=\left(u, q_{2}, q_{3} ; u m\right)$.

b) $e_{W}(h)=(m-1)\left(u m-q_{2}-q_{3}\right) / q_{2} q_{3}$,

c) $2 a_{0}=(m-1)\left(u m-q_{2}-q_{3}\right) / q_{2} q_{3}$,

d) $\nu_{W}=-(m-1)\left(u m-q_{2}-q_{3}-u q_{2} q_{3}\right) / q_{2} q_{3}$

$=-u m^{2} / q_{2} q_{3}+\left(u+u / q_{2} q_{3}+1 / q_{3}+1 / q_{2}\right) m-\left(u+1 / q_{2}+1 / q_{3}\right)$.

Type XIV.

a) $\bar{p}_{1}=\bar{p}_{2}=1, \bar{p}_{3} \neq 1, u=v=w=1, m \neq 1, q_{1}\left|m-q_{2}, q_{2}\right| m-q_{1}$,

$W=\left(q_{1} \bar{p}_{3}, q_{2} \bar{p}_{3}, q_{3} ; \bar{p}_{3} m\right)$.

b) $e_{W}(h)=\left(m-q_{1}\right)\left(m-q_{2}\right) / q_{1} q_{2} q_{3}, e_{W}\left(p_{1}\right)=-\left(m-q_{1}-q_{2}\right) / q_{2}$,

c) $2 a_{0}=m^{2} / q_{1} q_{2} q_{3}-\left(1 / q_{2} q_{3}+1 / q_{1} q_{3}+1 / q_{1} q_{2}\right) m+1 / q_{1}+1 / q_{2}+1 / q_{3}-1$,

d) $\nu_{W}=-m^{2} / q_{1} q_{2} q_{3}+\left(\bar{p}_{3}+1 / q_{2} q_{3}+1 / q_{1} q_{3}+\bar{p}_{3} / q_{1} q_{2}\right) m-\left(\bar{p}_{3} / q_{1}+\bar{p}_{3} / q_{2}+1 / q_{3}\right)$. 


\section{COROLlary 8.2 .}

i) $\quad \nu_{W} \begin{cases}\geq 0 & \text { if } m \leq \bar{p}_{1} \bar{p}_{2} \bar{p}_{3} q_{1} q_{2} q_{3}+\left(\bar{p}_{1} u q_{1}+\bar{p}_{2} v q_{2}+\bar{p}_{3} w q_{3}\right) / u v w-1 \\ <0 & \text { if } m \geq \bar{p}_{1} \bar{p}_{2} \bar{p}_{3} q_{1} q_{2} q_{3}+\left(\bar{p}_{1} u q_{1}+\bar{p}_{2} v q_{2}+\bar{p}_{3} w q_{3}\right) / u v w .\end{cases}$

ii) If $\nu_{W}=0$, then either following a) or b) holds.

a) $m=1$ and $W$ is either of types IV, VIII, XII, XIII or of type $V$ with $q_{i}=1{ }^{\exists} i$, type XI with $q_{3}=1$, type XIV with $q_{1}$ or $q_{2}=1$,

b) $m=\left\{\bar{p}_{1} \bar{p}_{2} \bar{p}_{3} q_{1} q_{2} q_{3}+\left(\bar{p}_{1} u q_{1}+\bar{p}_{2} v q_{2}+\bar{p}_{3} w q_{3}\right) / u v w-1\right\}$, where $\{x\}:=$ the least integer non less than $x$.

Proof. We regard $\nu_{W}$ as if it were a polynomial in $m$ of degree 2 by the expression (8.4). Then the corollary is proven if we show that $\left.\nu_{W}\right|_{m=0}<0$ and $\left.\nu_{W}\right|_{m=1} \geq 0$. In fact,

$$
\begin{aligned}
\left.\nu_{W}\right|_{m=0}= & -\left(\bar{p}_{2} \bar{p}_{3} u / q_{1}+\bar{p}_{1} \bar{p}_{3} v / q_{2}+\bar{p}_{1} \bar{p}_{2} w / q_{3}\right) \text { and } \\
\left.\nu_{W}\right|_{m=1}= & \bar{p}_{1} \bar{p}_{2} \bar{p}_{3} u v w-\left(\bar{p}_{2} \bar{p}_{3} u / q_{1}+\bar{p}_{1} \bar{p}_{3} v / q_{2}+\bar{p}_{1} \bar{p}_{2} w / q_{3}\right) \\
& +\left(\bar{p}_{1} u / q_{2} q_{3}+\bar{p}_{2} v / q_{1} q_{3}+\bar{p}_{3} w / q_{1} q_{2}\right)-u v w / q_{1} q_{2} q_{3} \\
= & (u-1 / u) \cdot \bar{p}_{1} / q_{2} q_{3}+(v-1 / v) \cdot \bar{p}_{2} / q_{3} q_{1}+(w-1 / w) \cdot \bar{p}_{3} / q_{1} q_{2} \\
& \left.+u v w\left[\bar{p}_{1} q_{1}-1 / v w\right)\left(\bar{p}_{2} q_{2}-1 / w u\right)\left(\bar{p}_{3} q_{3}-1 / u v\right)-1\right] / q_{1} q_{2} q_{3} \\
& +1 / u v w q_{1} q_{2} q_{3} .
\end{aligned}
$$

Apparently, $\left.\nu_{W}\right|_{m=0}$ is negative. To show the non-negativity of $\left.\nu_{W}\right|_{m=1}$, we need some works. In the second expression of $\left.\nu_{W}\right|_{m=1}$, only the second term could possibly be negative. Let us list all such cases, and check $\left.\nu_{W}\right|_{m=1} \geq 0$ in all cases.

i) case $\left(\bar{p}_{1} q_{1}-1 / v w\right)\left(\bar{p}_{2} q_{2}-1 / w u\right)\left(\bar{p}_{3} q_{3}-1 / u v\right)=0$.

This is the case either $\bar{p}_{1}=q_{1}=v=w=1, \bar{p}_{2}=q_{2}=w=u=1$ or $\bar{p}_{3}=q_{3}=u=$ $v=1$ (in fact, this happens for the types IV, XII, XIII and some special cases of type $\mathrm{V}$ and XIV). It is easy to check directly that in all those cases, one has $\left.\nu_{W}\right|_{m=1}=0$.

ii) case $0<\left(\bar{p}_{1} q_{1}-1 / v w\right)\left(\bar{p}_{2} q_{2}-1 / w u\right)\left(\bar{p}_{3} q_{3}-1 / u v\right)<1$.

a) If $u=v=w=1$, then $\bar{p}_{i} q_{1} \geq 2$ for all $i$ and hence $\left.\nu_{W}\right|_{m=1}>0$,

b) If $u>1, v=w=1$, then $\bar{p}_{1} q_{1}>1$. This is possible only for type III.

One has $q_{1}=1$ and $\left.\nu_{W}\right|_{m=1}=\left(\bar{p}_{1}-1\right)\left(u\left(q_{2}-1\right)\left(q_{3}-1\right)+(u-1)\left(q_{2}+q_{3}\right)\right)>0$. c) If $u, v>1, w=1$, then at least two of $\bar{p}_{i} q_{i}$ are 1 . All are 1 only for the type XI. Then $\left.\nu_{W}\right|_{m=1}=(u-1)(v-1)\left(1-1 / q_{3}\right) \geq 0$ and $=0$ only when $q_{3}=1$. Remaining cases are of type IX and X. Then $\left.\nu_{W}\right|_{m=1}=(u-1)\left(v\left(\bar{p}_{1}-1 / q_{3}\right)+\bar{p}_{1} / q_{3}-1\right)>0$, or $\left.\nu_{W}\right|_{m=1}=(v-1)(u-1)\left(\bar{p}_{3}-1 / q_{3}\right)>0$, respectively.

d) If $u, v, w>1$, then $\bar{p}_{i} q_{i}=1$ for all $i$. This is possible only for type VIII and then $\left.\nu_{W}\right|_{m=1}=0$.

REMARK 8.3. The corollary i) does not state about case when $m$ lies in the interval $\left(\bar{p}_{1} \bar{p}_{2} \bar{p}_{3} q_{1} q_{2} q_{3}+\left(\bar{p}_{1} u q_{1}+\bar{p}_{2} v q_{2}+\bar{p}_{3} w q_{3}\right) / u v w-1, \bar{p}_{1} \bar{p}_{2} \bar{p}_{3} q_{1} q_{2} q_{3}\left(\bar{p}_{1} u q_{1}+\bar{p}_{2} v q_{2}+\right.\right.$ $\left.\left.\bar{p}_{3} w q_{3}\right) / u v w\right)$. The corollary ii) b) states only the necessity for $\nu_{W}=0$ but not the sufficiency.

9. Discriminants $d(W)$ and $d^{*}(W)$. We introduce the discriminant $d(W)$ and the dual discriminant $d^{*}(W)$ for a weight system $W$ as follows.

$$
\begin{aligned}
d(W) & :=\prod_{i \in M(W)}(i)^{e_{W}(i)}, \\
d^{*}(W) & :=\prod_{i \in M(W)}(h / i)^{-e_{W}(i)} .
\end{aligned}
$$


They will be used in the product formula (10.4) for the signature of $W$ and in the duality formula for eta-products (13.3) and (13.3)*. The goal of this section is to prove that $d(W)$ and $d^{*}(W)$ are integers.

The next formula follows immediately from the definition.

$$
d(W)=h^{2 a_{0}} \cdot d^{*}(W) .
$$

Proof. $d(W) / d^{*}(W)=\prod_{i \in M(W)}(h)^{e_{W}(i)}=h^{2 a_{0}}$.

This formula, in particular, implies that square free factors of $d(W)$ and $d^{*}(W)$ coincide. Furthermore, it implies the next fact.

ASSERTION 9.1. If $W$ and $W^{*}$ are dual weight systems. Then,

$$
d(W)=d\left(W^{*}\right)=d^{*}(W)=d^{*}\left(W^{*}\right)
$$

Proof. The definitions of $d\left(W^{*}\right)$ and $d^{*}(W)$ coincides (see (7.7)). Recall that $a_{0}=0$ if the weight system $W$ has its dual (see $\S 7$ Assertion $7.71 \mathrm{i}$ )).

The main goal of the present section is the next theorem.

ThEOREM 9.2. The discriminants $d(W)$ and $d^{*}(W)$ are integers.

Proof. The theorem is a corollary of the next theorem, where we give a formula (9.5) of the dual discriminant in terms of refined coordinate $\left(\bar{p}_{1}, \bar{p}_{2}, \bar{p}_{3}, u, v, w, m, q_{1}, q_{2}\right.$, $\left.q_{3}\right)$ for $W$ (recall (8.1)-(8.3)). We have only to notify that the exponents $\left(u m-q_{2}-q_{3}+\right.$ $\left.q_{2} q_{3}\right) / q_{2} q_{3}$ etc. in the formula (9.5) are non-negative (obvious from the expression) and integral (for they are, by definition (9.2), integral linear combinations of the cyclotomic exponents, depending on the type of $W)$. For $d(W)$ use (9.3).

TheOREM 9.3. Let $W$ be a weight system. One has

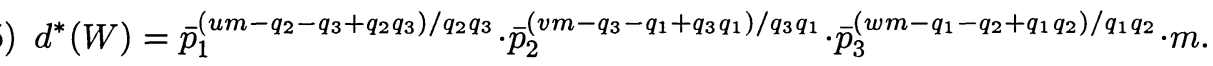

Proof. The proof depends on the type of $W$. In the following, we calculate $d^{*}(W)$ for each type according to the data given in $\S 8$ Table G. It turns out that the resulting expression of $d^{*}(W)$ is a specialization of the formula (9.5). Details of the verifications are left to the reader. We check the condition for $d^{*}(W)$ to be equal to 1 in order to prove theorem-bis.

Type I. One has $\bar{p}_{1} \neq 1, \bar{p}_{2} \neq 1, \bar{p}_{3} \neq 1$ and $q_{1}=q_{2}=q_{3}=1$. Then,

$$
\begin{aligned}
d^{*}(W) & :=\bar{p}_{1}^{u m} \cdot \bar{p}_{2}^{v m} \cdot \bar{p}_{3}^{w m} \cdot \bar{p}_{1} \bar{p}_{2} \bar{p}_{3} u v w m /\left(1 \cdot \bar{p}_{2} \bar{p}_{3} u \cdot \bar{p}_{3} \bar{p}_{1} v \cdot \bar{p}_{1} \bar{p}_{2} w\right) \\
& =\bar{p}_{1}^{u m-1} \cdot \bar{p}_{2}^{v m-1} \cdot \bar{p}_{3}^{w m-1} \cdot m .
\end{aligned}
$$

This equals 1 if and only if $m=u=v=w=1$.

Type II. One has $\bar{p}_{2}=w=1, \bar{p}_{1} \neq 1, \bar{p}_{3} \neq 1, u \neq 1$ and $q_{1}=q_{2}=1$. Then,

$$
\begin{aligned}
d^{*}(W) & :=\bar{p}_{1}^{(u m-1) / q_{3}} \cdot \bar{p}_{3}^{m} \cdot \bar{p}_{1} \bar{p}_{3} u v m /\left(1 \cdot \bar{p}_{3} u \cdot \bar{p}_{3} \bar{p}_{1} v\right) \\
& =\bar{p}_{1}^{(u m-1) / q_{3}} \cdot \bar{p}_{3}^{m-1} \cdot m .
\end{aligned}
$$

This can never be equal to 1 .

Type III. One has $\bar{p}_{2}=\bar{p}_{3}=v=w=1, \bar{p}_{1} \neq 1, u \neq 1$ and $q_{1}=1$. Then,

$$
\begin{aligned}
d^{*}(W) & :=\bar{p}_{1}^{\left(u m-q_{2}-q_{3}\right) / q_{2} q_{3}} \cdot \bar{p}_{1} u m /(1 \cdot u) \\
& =\bar{p}_{1}^{\left(u m-q_{2}-q_{3}\right) / q_{2} q_{3}} \cdot \bar{p}_{1} \cdot m .
\end{aligned}
$$

This can never be equal to 1 . 
Type IV. One has $\bar{p}_{1}=\bar{p}_{2}=v=w=1, \bar{p}_{3} \neq 1, u \neq 1, m \neq 1$ and $q_{1}=1$. Then,

$$
d^{*}(W):=\bar{p}_{3}^{(m-1) / q_{2}} \cdot \bar{p}_{3} u m /\left(1 \cdot \bar{p}_{3} u\right)=\bar{p}_{3}^{(m-1) / q_{2}} \cdot m .
$$

This can never be equal to 1 .

Type V. One has $\bar{p}_{1}=\bar{p}_{2}=\bar{p}_{3}=u=v=w=1, m \neq 1$. Then,

$$
d^{*}(W):=m \text {. }
$$

This can never be equal to 1 .

Type VI. One has $\bar{p}_{2}=1, \bar{p}_{1} \neq 1, \bar{p}_{3} \neq 1, u \neq 1, w \neq 1$ and $q_{1}=q_{2}=q_{3}=1$. Then,

$$
\begin{aligned}
d^{*}(W) & :=\bar{p}_{3}^{w m} \cdot \bar{p}_{1}^{u m} \cdot \bar{p}_{1} \bar{p}_{3} u v w m /\left(\bar{p}_{3} u \cdot \bar{p}_{1} \bar{p}_{3} v \cdot \bar{p}_{1} w\right) \\
& =\bar{p}_{3}^{w m-1} \cdot \bar{p}_{1}^{u m-1} \cdot m .
\end{aligned}
$$

This can never be equal to 1 .

Type VII. One has $\bar{p}_{2}=\bar{p}_{3}=1, \bar{p}_{1} \neq 1, u \neq 1, v \neq 1, w \neq 1$ and $q_{1}=q_{2}=q_{3}=1$.

Then

$$
d^{*}(W):=\bar{p}_{1}^{u m} \cdot \bar{p}_{1} u v w m /\left(u \cdot \bar{p}_{1} v \cdot \bar{p}_{1} w=\bar{p}_{1}^{u m-1} \cdot m\right) .
$$

This can never be equal to 1 .

Type VIII. One has $\bar{p}_{1}=\bar{p}_{2}=\bar{p}_{3}=1, u \neq 1, v \neq 1, w \neq 1$. Then

$$
d^{*}(W):=u v w m /(u \cdot v \cdot w)=m .
$$

This is equal to 1 if and only if $m=1$.

Type IX. One has $\bar{p}_{2}=\bar{p}_{3}=w=1, \bar{p} \neq 1, u \neq 1, v \neq 1$ and $q_{1}=q_{2}=1$.

$$
d^{*}(W):=\bar{p}_{1}^{(u m-1) / q_{3}} \cdot \bar{p}_{1} u v m /\left(u \cdot \bar{p}_{1} v\right)=\bar{p}_{1}^{(u m-1) / q_{3}} \cdot m .
$$

This can never be equal to 1 .

Type X. One has $\bar{p}_{1}=\bar{p}_{2}=w=1, \bar{p}_{3} \neq 1, u \neq 1, v \neq 1$ and $q_{1}=q_{2}=1$. Then,

$$
d^{*}(W):=\bar{p}_{3}^{m} \cdot \bar{p}_{3} u v m /\left(\bar{p}_{3} u \cdot \bar{p}_{3} v\right)=\bar{p}_{3}^{m-1} \cdot m \text {. }
$$

Then $d^{*}(W)=1$ if and only if $m=1$.

Type XI. One has $\bar{p}_{1}=\bar{p}_{2}=\bar{p}_{3}=w=1, u \neq 1, v \neq 1$. Then

$$
d^{*}(W):=u v m /(u \cdot v)=m .
$$

Then $d^{*}(W)=1$ if and only if $m=1$.

Type XII. One has $\bar{p}_{1}=v=w=1, \bar{p}_{2} \neq 1, \bar{p}_{3} \neq 1, m \neq 1$ and $q_{1}=1$. Then,

$$
\begin{aligned}
d^{*}(W) & :=\bar{p}_{3}^{(m-1) / q_{2}} \cdot \bar{p}_{2}^{(m-1) / q_{3}} \cdot \bar{p}_{2} \bar{p}_{3} u m /\left(u \bar{p}_{2} \bar{p}_{3}\right) \\
& =\bar{p}_{3}^{(m-1) / q_{2}} \cdot \bar{p}_{2}^{(m-1) / q_{3}} \cdot m .
\end{aligned}
$$

This can never be equal to 1 . 
Type XIII. One has $\bar{p}_{1}=\bar{p}_{2}=\bar{p}_{3}=v=w=1$ and $u \neq 1, m \neq 1$. Then,

$$
d^{*}(W):=u \cdot m / u=m .
$$

This can never be equal to 1 .

Type XIV. One has $\bar{p}_{1}=\bar{p}_{2}=u=v=w=q_{1}$ and $\bar{p}_{3} \neq 1, m \neq 1$. Then

$$
d^{*}(W):=\bar{p}_{3}^{\left(m-q_{1}-q_{2}\right) / q_{1} q_{2}} \cdot p_{3} \cdot m .
$$

This can never be equal to 1 .

This completes the proof of Theorem 9.3.

THEOREM 9.4. In the following, we give the list of weight system $W$ with $d^{*}(W)=$ 1. There are 4 types: type I, VIII, $X$ and $X I$.

Among them, only the type $I$ admits $a_{0}=0$, and hence, $d(W)=1$.

1. Type $I: \quad W:=\left(\bar{p}_{2} \bar{p}_{3}, \bar{p}_{3} \bar{p}_{1}, \bar{p}_{1} \bar{p}_{2} ; \bar{p}_{1} \bar{p}_{2} \bar{p}_{3}\right)$

for relatively prime integers $\bar{p}_{1}, \bar{p}_{2}$ and $\bar{p}_{3}>1$. Then, $e_{W}(h)=1$ and $\varphi_{W}=\left(\lambda^{u v w}-\right.$ 1) $\left(\lambda^{u}-1\right)\left(\lambda^{v}-1\right)\left(\lambda^{w}-1\right) /(\lambda-1)\left(\lambda^{v w}-1\right)\left(\lambda^{w u}-1\right)\left(\lambda^{u v}-1\right)$. So $a_{0}=0$ and $\mu=\nu=\left(\bar{p}_{1}-1\right)(v-1)(w-1)>0$.

2. Type VIII: $\quad W:=(u, v, w ; u v w)$

for relatively prime integers $u, v$ and $w(>1)$. Then $e_{W}(h)=u v w-u-v-w$ and $\varphi_{W}=\left(\lambda^{u v w}-1\right)^{e(h)}\left(\lambda^{u v}-1\right)\left(\lambda^{v w}-1\right)\left(\lambda^{w u}-1\right) /(\lambda-1)$. So $a_{0}=u v w-u-v-w+2>0$ and $\mu=(u v-1)(v w-1)(w u-1), \nu=u v w-u-v-w-1>0$.

3. Type $X . \quad W:=(u m, v m, w ; u v m)$

for relatively prime integers $u, v(>1)$ and $w$ and an integer $m>1$ with $w \mid u-1$ or $w \mid v-1,(w, m)=1$. Then, $e_{W}(h)=(u-1)(v-1) / w$ and $\varphi_{W}=\left(\lambda^{u v m}-\right.$ $1)^{e(h)}\left(\lambda^{u}-1\right)\left(\lambda^{v}-1\right) /(\lambda-1)\left(\lambda^{u v}-1\right)$. So $a_{0}=(u-1)(v-1) / w>0$ and $\mu=$ $(u-1)(v-1)(u v m-1) / w, \nu=(u-1)(v-1) m-1>0$.

\section{Type $X I . \quad W:=(u, v, w ; u v)$}

for relatively prime integers $u, v(>1)$ and $w$ with $w \mid u-1$ or $w \mid v-1$. Then $e_{W}(h)=(u-1)(v-1) / w-1$ and $\varphi_{W}=\left(\lambda^{u v}-1\right)^{e(h)-1}\left(\lambda^{u}-1\right)\left(\lambda^{v}-1\right) /(\lambda-1)$. So $a_{0}=(u-1)(v-1) / w>0$ and $\mu=(u-1)(v-1)(u v-w) / w, \nu=u v-u-v-1 \geq 0$.

REMARK 9.5. 1. It is a bit surprise to observe that the factors in the powers of $u, v$ and $w$ are canceled out in the formula (9.5) of the discriminant. The author does not know a significance of this fact.

2. The formulae (8.4), (8.5) and (9.5) seem to suggest as if there exists a virtual dual weight system $W^{*}$ for any $W$ so that the formulae describe universally the numerical invariants of $W^{*}$. What is the natural category, which contains weight systems as its subcategory and is closed under the duality operation $*$ ?

10. Signature $A(W)$ of a weight system. We attach to a weight system $W$ a finite set of integers $A(W)$, which we call the signature of $W$ (the name has an origin in the signature for a Fuchsian group, see remarks at the end of this section). The numbers will be identified with the Dolgachev numbers for the 14 unimodular exceptional singularities in $\S 13$ in case of weight system with $\varepsilon_{W}=-1$. The goal of this section is to show a product formula (10.4) for the signature.

First we fix notation. Let $A$ and $B$ be sets of finite positive integers, where the same number may appear multiply. We denote $A \equiv B$ if any integer, except for 1 , appears in $A$ and $B$ with the same multiplicity. The "三" obviously 
defines an equivalence relation. Put $\Pi A:=\prod_{p \in A} \quad p$ and $\#^{\prime} A:=$ the number of elements of $A$ which is not equal to 1 . By $u * v$ we denote $v$ times copy of $u$. For instance, $\{1,2,3,1,2,3\} \equiv\{2,2,3,3\}=\{2 * 2,3 * 2\}, \#^{\prime}\{1,2,3,1,2,3\}=4$ and $\Pi\{1,2,3,1,2,3\}=36$.

To a weight system $W=(a, b, c ; h)$, we attach two sets of integers:

$A(W):=\left\{a_{i}: a_{i} \nmid h, 1 \leq i \leq 3\right\} \amalg\left\{\operatorname{gcd}\left(a_{i}, a_{j}\right) *\left(m\left(a_{i}, a_{j} ; h\right)-1\right): 1 \leq i<j \leq 3\right\}$,

$$
\widetilde{A}(W):=\left\{a_{i}: n\left(p_{i}\right) \neq 1,1 \leq i \leq 3\right\} \amalg\left\{(h / p) *\left(-e_{W}(p)\right) \mid p \in M(W), n(p)=2\right\} .
$$

Here, $a_{1}:=a, a_{2}=b$ and $a_{3}:=c$, and $m(k, l ; h):=\#\left\{(u, v) \in \mathbb{Z}_{\geq 0}^{2} \mid h=u k+v l\right\}$. We will show the equivalence: $A(W) \equiv \widetilde{A}(W)$, and the equivalence class will be called the signature of $W$.

THEOREM 10.1. Let $W=(a, b, c ; h)$ be a primitive weight system, which is not of type $A_{\ell}$. Then

$$
\begin{gathered}
A(W) \equiv \widetilde{A}(W) . \\
\Pi A(W)=\Pi \widetilde{A}(W)=a \cdot b \cdot c \cdot d(W) / h^{1+2 a_{0}}=a \cdot b \cdot c \cdot d^{*}(W) / h .
\end{gathered}
$$

Proof. Decompose $A(W)=B_{1} \amalg B_{2}$ and $\widetilde{A}(W)=\widetilde{B}_{1} \amalg \widetilde{B}_{2}$, where $B_{1}:=\left\{a_{i}\right.$ : $\left.a_{i} \nmid h, 1 \leq i \leq 3\right\}, B_{2}:=\left\{\operatorname{gcd}\left(a_{i}, a_{j}\right) *\left(m\left(a_{i}, a_{j} ; h\right)-1\right): 1 \leq i<j \leq 3\right\}, \widetilde{B}_{1}:=\left\{a_{i}:\right.$ $\left.n\left(p_{i}\right) \neq 1,1 \leq i \leq 3\right\}$ and $\widetilde{B}_{2}:=\left\{(h / p) *\left(-e_{W}(p)\right) \mid p \in M(W), n(p)=2\right\}$. The proof of (10.3) is done by the following 3 steps:

1. $B_{1} \subset \widetilde{B}_{1}$. 2. $\widetilde{B}_{2} \subset B_{2} . \quad$ 3. $\left(\widetilde{B}_{1} \backslash B_{1}\right) \equiv\left(B_{2} \backslash \widetilde{B}_{2}\right)$.

Proof of 1 . Since $h / a_{i}=p_{i} / q_{i}$, we may rewrite $B_{1}=\left\{a_{i}: q_{i} \neq 1,1 \leq i \leq 3\right\}$. The fact that $n\left(p_{i}\right)=1$ implies $q_{i}=1$ ( $\S 5$ Assertion 5.3 ii)), implies $B_{1} \subset \widetilde{B}_{1}$.

Proof of 2. Take any element $p \in M(W)$ with $n(p)=2$. Without a loss of generality, we assume that $p=p_{12}=1 \mathrm{~cm}\left(p_{1}, p_{2}\right)$. We have $a_{1}=\left(h, a_{1}\right) q_{1}, a_{2}=\left(h, a_{2}\right) q_{2}$ and $h=\left(h, a_{1}\right) p_{1}=\left(h, a_{2}\right) p_{2}$. Note that $\left(h, a_{1}\right) /\left(h, a_{1}, a_{2}\right)$ and $q_{2}$ are co-prime, otherwise the common factor in $q_{2}$ still devises $h /\left(h, a_{2}\right)$, which contradicts to the definition of $q_{3}$. Similarly, $\left(h, a_{2}\right) /\left(h, a_{1}, a_{2}\right)$ and $q_{1}$ are co-prime. Therefore $\left(a_{1}, a_{2}\right)=$ $\left(h, a_{1}, a_{2}\right) \cdot\left(q_{1}, q_{2}\right)$. Since $\left(q_{1}, q_{2}\right)=1(4.8)$, we have $\left(a_{1}, a_{2}\right)=\left(h, a_{1}, a_{2}\right)$. Therefore, $h / p=h / 1 c m\left(p_{1}, p_{2}\right)=\operatorname{gcd}\left(h / p_{1}, h / p_{2}\right)=\operatorname{gcd}\left(\left(h, a_{1}\right),\left(h, a_{2}\right)\right)=\left(h, a_{1}, a_{2}\right)=\left(a_{1}, a_{2}\right)$. We need to show that $-e_{W}(p)<m\left(a_{1}, a_{2} ; h\right)$. Actually, this will be proven in the next step 3.

Proof of 3. First, note that if $n\left(p_{i}\right)=3$ and $q_{i}=1$, then by definition $a_{i}=$ $q_{i} h / p_{i}=1$. Therefore, $\widetilde{B}_{1} \backslash B_{1} \equiv B_{1}^{*}:=\left\{a_{i}: n\left(p_{i}\right)=2\right.$ and $\left.q_{i}=1,1 \leq i \leq 3\right\}$. On the other hand, let us show that if $n\left(p_{i j}\right) \neq 2$ for $1 \leq i, j \leq 3$ with $i \neq j$, then either $\left(a_{i}, a_{j}\right)=1$ or $m\left(a_{i}, a_{j} ; h\right) \leq 1$. By assumption $n\left(p_{i j}\right)=3$ and hence $h=$ $p_{i j}=1 \mathrm{~cm}\left(h /\left(h, a_{i}\right), h /\left(h, a_{j}\right)\right)=h /\left(h, a_{i}, a_{j}\right)$. So $\left(h, a_{i}, a_{j}\right)=1$ and hence $\left(a_{i}, a_{j}\right)=$ $\left(h, a_{i}, a_{j}\right) \cdot\left(q_{i}, q_{j}\right)=\left(q_{i}, q_{j}\right)$ and $h=\left(h, a_{i}\right)\left(h, a_{j}\right) k$ for some $k$. If $\left(q_{i}, q_{j}\right) \neq 1$, then $h \notin\left(q_{i}, q_{j}\right)$ by definition of $q_{i}$ (and $\left.q_{j}\right)$. So $h \notin\left(a_{i}, a_{j}\right)$ and therefore $m\left(a_{i}, a_{j} ; h\right)=0$. This implies that $B_{2} \backslash \widetilde{B}_{2} \equiv B_{2}^{*}:=\left\{\left(a_{i}, a_{j}\right) *\left(m\left(a_{i}, a_{j} ; h\right)-1-e_{W}\left(p_{i j}\right)\right.\right.$ for $1 \leq i<j \leq 3$ with $\left.n\left(p_{i j}\right)=2\right\}$. So, let us prove $B_{1}^{*} \equiv B_{2}^{*}$.

Let $p \in M(W)$ and $n(p)=2$. Assume $p=p_{12}$. It is sufficient to show

$$
m\left(a_{1}, a_{2} ; h\right)=1-e_{W}(p)+\#\left\{1 \leq i \leq 3 \mid p_{i}=p \quad \text { and } q_{i}=1\right\} .
$$


The proof is separated according to the type of the poset $M_{p}:=\{\eta \in M(W)|\eta| p\}$. There are 3 types to consider (cf. the proof of Theorem 5.1).

i) $M_{p}=1$. In this case, $q_{1}=q_{2}=1$ but $p \neq p_{12}$ and $p \neq p_{2}$. Since $h=p_{1} a=p_{2} b$, there exists $d \in \mathbb{N}$ such that $h=d p_{1} p_{2} /\left(p_{1}, p_{2}\right)$, $a=d p_{2} /\left(p_{1}, p_{2}\right)$ and $b=d p_{1} /\left(p_{1}, p_{2}\right)$. So $m(a, b ; h)=m\left(p_{1} /\left(p_{1}, p_{2}\right), p_{2} /\left(p_{1}, p_{2}\right)\right.$; $\left.p_{1} p_{2} /\left(p_{1}, p_{2}\right)\right)=\left(p_{1}, p_{2}\right)+1=p_{1} p_{2} / p_{12}+1$, which is equal to $1-e_{W}(p)$ (cf. (5.2)).

ii) $M_{p}=$ (1) $p=p_{2}=p_{12}$. This case $q_{1}=1$ but $p \neq p_{1}$. Since $h=p_{1} a=p_{2}(h, b)$, one has $p_{2} / p_{1}=a /(h, b)=: d$ and $h=p_{1} d(h, b), a=d(h, b)$ and $b=q_{2}(h, b)$, where we note that $q_{2}$ is prime to $p_{2}$ and hence to $d$. Since we know that $q_{2} \mid p_{1}-1$, one has $m(a, b ; h)=m\left(d, q_{2} ; p_{1} d\right)=1+\left(p_{1}-1\right) / q_{2}+\delta\left(q_{2}-1\right)=$ $1-e_{W}(p)+\#\left\{1 \leq i \leq 3 \mid p_{i}=p\right.$ and $\left.q_{i}=1\right\}$ (cf. (5.3)), where $\delta(x)$ is either equal to 1 or 0 according as $x=0$ or not.

iii) $M_{p}=$ (1) $p=p_{1}=p_{2}=p_{12}$. Since $p=p_{1}=p_{2}$, one has $(h, a)=$ $(h, b)=h / p$ which we denote by $d$. Then $h=d p, a=d q_{1}$, and $b=d q_{2}$, where $\left(q_{1}, q_{2}\right)=1$ (cf. the proof of step 2). Since we know $q_{1} \mid p-q_{2}$ and $q_{2} \mid p-q_{1}$ (cf. $\S 5$ Assertion iii)), one has $q_{1} q_{2} \mid p-q_{1}-q_{2}$. Therefore $m(a, b ; h)=m\left(q_{1}, q_{2} ; p\right)=$ $1+\left(p-q_{1}-q_{2}\right) / q_{1} q_{2}+\#\left\{1 \leq i \leq 2 \mid q_{i}=1\right\}=1-e_{W}(p)+\#\left\{1 \leq i \leq 3 \mid p_{i}=p, q_{i}=1\right\}$ (cf. $(5.4)$ ).

This completes a proof of (10.3).

$A$ proof of (10.4). Since $a=q_{1} h / p_{1}, b=q_{2} h / p_{2}$ and $c=q_{3} h / p_{3}$, one has $a \cdot b \cdot c=$ $q_{1} q_{2} q_{3} h^{3} / p_{1} p_{2} p_{3}$. Therefore the right hand side of (10.4) is equal to

$$
a \cdot b \cdot c \cdot d(W) / h^{1+2 a_{0}}=\frac{q_{1} q_{2} q_{3}}{p_{1} p_{2} p_{3}} h^{2} d(W) / h^{2 a_{0}}=\frac{q_{1} q_{2} q_{3}}{p_{1} p_{2} p_{3}} h^{2} \prod_{p \in M(W)}(p / h)^{e_{W}(p)} .
$$

Decompose the last factor into $\prod_{j=1}^{3}\left(\prod_{n(p)=j}(p / h)^{e_{W}(p)}\right)$. The factor for $j=0$ or 3 is equal to $h$ or 1 , respectively. If $n(p)=1$, then $p=p_{i}$ for $1 \leq i \leq 3$. Recalling the facts $e_{W}\left(p_{i}\right)=1$ and $q_{i}=1$ for $n\left(p_{i}\right)=1$ (Theorem 5.12 and Assertion $5.3 \mathrm{ii}$ ), we write the formula as:

$$
=\prod_{\substack{1 \leq i \leq 3 \\ n\left(p_{i}\right) \neq 1}}\left(q_{i} \frac{h}{p_{i}}\right) \cdot \prod_{\substack{p \in M(W) \\ n(p)=2}}\left(\frac{h}{p}\right)^{-e_{W}(p)}=\Pi \widetilde{B}_{1} \cdot \Pi \widetilde{B}_{2}=\Pi \widetilde{A}(W)=\Pi A(W) .
$$

This completes a proof of (10.4).

COROLlary 10.2 .

1. Under the same setting as in Theorem 8.1, one has

$$
\#^{\prime} A(W)=2+\operatorname{mult}(W)-2 a_{0}-\#\left\{1 \leq i \leq 3 \mid n\left(p_{i}\right) \neq 1, q_{i}=1\right\} .
$$

2. Suppose $W$ is simple (cf. $\S 6$ Theorem 6.3). Then

$$
\#^{\prime} A(W)= \begin{cases}3 & \text { if } a_{0}=0 \text { and } W \text { is of dual type } \\ 1 & \text { if } a_{0}=1 \text { and } W \text { is of type } I X \text { and } k>0 \\ 0 & \text { if } a_{0}=1 \text { and } W \text { is of type } I X \text { and } k=0\end{cases}
$$


The set $A(W)$ is given as follows.

I. $\left\{p_{1}, p_{2}, p_{3}\right\}=\{h / a, h / b, h / c\}$.

II. $\left\{p_{1}, p_{3} / p_{2},\left(p_{2}-1\right) p_{1}\right\}$.

III. $\left\{p_{1}, p_{1} q_{2}, p_{1} q_{3}\right\}$.

$I V .\left\{p_{3} / p_{2},\left(p_{1}-1\right) p_{3} / p_{2}, p_{2}-p_{1}+1\right\}$.

$V .\left\{q_{1}, q_{2}, q_{3}\right\}=\{a, b, c\}$.

$X I .\left\{q_{3}\right\}=\{2 k+1\},\{3 k+1\}$ or $\{4 k+1\}$ according as $W$ is $(6.22,23$ or 24$)$.

Proof. 1. From the expression (10.2) for $\widetilde{A}(W)$ and (10.4) one has

$$
\begin{aligned}
\#^{\prime} A(W) & =\#\left\{1 \leq i \leq 3 \mid n\left(p_{i}\right) \neq 1, q_{i} \neq 1\right\}-\sum_{\substack{p \in M(W) \\
n(p)=2}} e_{W}(p) \\
& =3-\sum_{\substack{p \in M(W) \\
1 \leq n(p) \leq 2}} e_{W}(p)-\#\left\{1 \leq i \leq 3 \mid n\left(p_{i}\right) \neq 1, q_{i}=1\right\},
\end{aligned}
$$

which implies the formula (10.5) (cf. (7.5) and (3.4)).

2. The (10.5) implies that $a_{0}$ is at most 1. Then $W$ is either dual type for $a_{0}=0$ or type IX for $a_{0}=1$ (see Theorem 6.3).

REMARK 10.3.

1. Some particular cases of (10.4) were known ([D], [O-W], [S5], [S6]).

2. The signature $A(W)$ has an origin in a study of normal surface singularity with a $\mathbb{C}^{\times}$-action ([D1-2], [O-W], [P2-3], [S7, (5.6.5)]). Let a weight system $W=$ $(a, b, c ; h)$ be given. Consider an action of $t \in \mathbb{C}^{\times}$on $(x, y, z) \in \mathbb{C}^{3}$ by $t \cdot(x, y, z):=$ $\left(t^{a} x, t^{b} y, t^{c} z\right)$ and define a weighted projective plane $\mathbb{P}(a, b, c):=\left\{\mathbb{C}^{3} \backslash\{0\}\right\} / \mathbb{C}^{\times}$. A curve $C$ in $\mathbb{P}(a, b, c)$ of degree $h$ is defined by $a$ weighted homogeneous polynomial $f_{W}(x, y, z)=\sum_{a i+b j+c k=h} c_{i j k} x^{i} y^{j} z^{k}$.

For a generic choice of the coefficients of $f_{W}$, the curve $C_{W}$ is smooth if and only if the weight system $W:=(a, b, c ; h)$ is regular, and its genus is given by $a_{0}$ ([S6, theo. 3]). Consider the surface $X_{W, 0}:=\left\{(x, y, z) \in \mathbb{C}^{3} \mid f_{W}(x, y, z)=0\right\}$ with the natural projection $X_{W, 0} \backslash\{0\} \rightarrow C_{W}$ by $\mathbb{C}^{\times}$-action so that $C_{W}$ is an orbifold (where $X_{W, 0} \backslash\{0\}$ is smooth, for $C_{W}$ is smooth). Any singular orbit of $\mathbb{C}^{\times}$-action lies in the intersection of $C_{W}$ with coordinate axises $\ell_{x}, \ell_{y}$ and $\ell_{z}$ of $\mathbb{P}(a, b, c)$. There is a oneto-one correspondence between the set $A(W)$ and the set $C_{W} \cap\left(\ell_{x} \cup \ell_{y} \cup \ell_{z}\right)$, where the values of $A(W)$ describes the order of the isotropy groups at the points. The pair $\left(a_{0} ; A(W)\right)$ of the genus $a_{0}$ of $C_{W}$ and the set $A(W)$ of orders of isotropy groups, is called the signature of the orbifold $C_{W}$. In fact, $C_{W}$ (resp. $X_{W, 0}$ ) can be realized as the quotient of the Riemann sphere $\mathbb{P}$ for $\varepsilon_{W}>0$ or the complex upper half plane $\mathbb{H}$ for for $\varepsilon_{W}<0$ (resp. $-\varepsilon_{W}$-th root of the canonical bundle of $\mathbb{H}$ ) by the action of a Fuchsian group of the signature $\left(a_{0} ; A(W)\right)$ ([Mag,p98]) (resp. a lifting of the Fuchsian group in the $-\varepsilon_{W}$-th covering of $P S L(2, \mathrm{~B})([\mathrm{D} 1-3],[\mathrm{S} 7, \S 5])$.

3. The above corollary 2 . implies that if $W^{\circ}$ is simple,

$$
C_{W}=\left\{\begin{array}{l}
\text { a rational curve with } 3 \text { fixed points, if } W \text { is dual type } \\
\text { an elliptic curve with } 1 \text { fixed point, if } W \text { is type } I X, k>0 \\
\text { an elliptic curve without a fixed point, if } W \text { is type } \widetilde{E}_{8}
\end{array}\right.
$$

Let us give an explicit weighted homogeneous equation of $C_{W}$.

I. $x^{p_{1}}+y^{p_{2}}+z^{p_{3}}$ for $\left(p_{i}, p_{j}\right)=1 i \neq j$.

II. $x^{p_{1}}+y^{p_{2}}+y z^{p_{3} / p_{2}}$ for $p_{2} \neq p_{3},\left(p_{1}, p_{3}\right)=1,\left(p_{2}-1, p_{3}\right)=1$. 


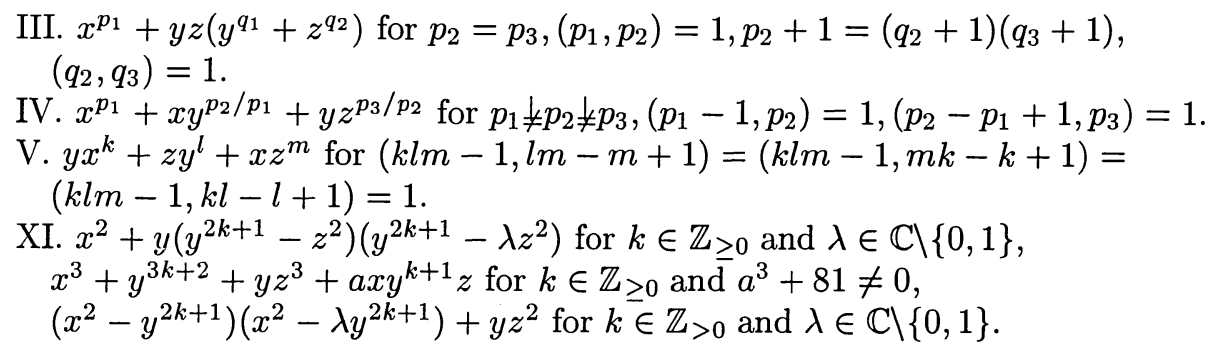

Note that except for the last type XI, each curve $C_{W}$ for the dual types I-V is the quotient of the sphere or the upper half-plane by the triangle groups (cf. [Mag, Chap.II])

11. The Cartan matrix of type $A D E$. First, we recall an observation of H. Ochiai [Oc] in its original form. Denote by [n] the function $q^{n}-q^{-n}$ in $q$. Let $C$ be the Cartan matrix of type either $A_{\ell}, D_{\ell}, E_{6}, E_{7}$ or $E_{8}$. Put $\Phi(q):=$ $\operatorname{det}\left(\frac{[2]}{[1]} I-C+2 I\right)$ where $I$ is the identity matrix. Then $\Phi$ decomposes into a finite product $\Pi_{i}[i / 2]^{d_{i}}$ for suitable integers $d_{i} \in \mathbb{Z}$. Then Ochiai's observation [Oc] states:

i) if $d_{i} \neq 0$, then $i \mid h$, where $h$ is the Coxeter number for $C$,

ii) $d_{i} \in\{-2,0,2\}$ for any $i \in \mathbb{N}$,

iii) if $i j=h$ for $i, j \in \mathbb{N}$, then $d_{i}+d_{j}=0$,

iv) $d_{i} \neq 0$ for all divisors $i$ of $h$ if $C$ is of type $D_{4}, E_{6}, E_{7}$ or $E_{8}$.

We translate the observation in terms of Coxeter element. Let Cox be a Coxeter matrix for the Cartan matrix $C$ and let $\varphi(\lambda):=\operatorname{det}(\lambda I-$ Cox $)$ be its characteristic polynomial. The eigenvalues of $\operatorname{Cox}$ are $\omega_{i}:=\exp \left(2 \pi \sqrt{-1} m_{i} / h\right)$ for the exponent $m_{i}, i=1, \cdots, \ell$. So $\varphi(\lambda)=\prod_{i=1}^{\ell}\left(\lambda-\omega_{i}\right)$. Decompose it to $\varphi(\lambda)=\prod_{i \mid h}\left(\lambda^{i}-1\right)^{e(i)}$ for some exponents $e(i) \in \mathbb{Z}$. Since $\operatorname{Cox}+\operatorname{Cox}^{-1}$ is conjugate to $C-2 I$, one has

$$
\begin{aligned}
\Phi(q) & =\operatorname{det}\left(\left(q+q^{-1}\right) I-\operatorname{Cox}-\operatorname{Cox}^{-1}\right)=\prod_{i=1}^{\ell}\left(q+q^{-1}-\omega_{i}-\omega_{i}^{-1}\right) \\
& =\prod_{i=1}^{\ell}\left(q-\omega_{i}\right)\left(q-\omega_{i}^{-1}\right) / q=q^{-\ell}\left(\prod_{i=1}^{\ell}\left(q-\omega_{i}\right)\right)^{2}=\varphi(q)^{2} / q^{\ell} \\
& =q^{-\ell} \prod_{i \mid h}\left(q^{i}-1\right)^{2 e(i)}=\prod_{i \mid h}\left(\left(q^{i}-1\right)^{2} / q^{i}\right)^{e(i)}=\prod_{i \mid h}\left(q^{i / 2}-1 / q^{i / 2}\right)^{2 e(i)} \\
& =\prod_{i \mid h}[i / 2]^{2 e(i)}
\end{aligned}
$$

where we used the fact $\sum i e(i)=l$. Thus one gets $d_{i}=2 e(i)$ for $i \in \mathbb{N}$. So, the Ochiai's observation is equivalent to the following statements.

i) $i \mid h$ for $e(i) \neq 0$,

ii) $e(i) \in\{0, \pm 1\}$ for any $i \in \mathbb{N}$,

iii) $e(i)+e(h / i)=0$ for $i \in \mathbb{N}$ i.e. $\varphi=\varphi^{*}$,

iv) $\operatorname{Div}(h)=\{i \in \mathbb{N}: e(i) \neq 0\}$ for any $D_{4}, E_{6}, E_{7}, E_{8}$.

These can be easily verified as follows. First, recall that there is a one to one correspondence between the root systems of types $A_{\ell}, D_{\ell}, E_{6}, E_{7}$ and $E_{8}$ and the weight systems having only positive exponents in such way that the set of exponents for them coincides. For such weight systems, one can prove the i)-iv) as follows. 
i) By definition, one has $M(W) \subset \operatorname{Div}(h)(\S 4)$.

ii) This is the simplicity of $W$ ( $\S 1$ Assertion 1.2 and $\S 6$ Theorem 6.2).

iii) This is the self-duality of $W(\S 7$ Theorem 7.101$)$.

iv) This is a consequence of the classification of weight systems with only positive exponents, recalled in Table B.

Table B.

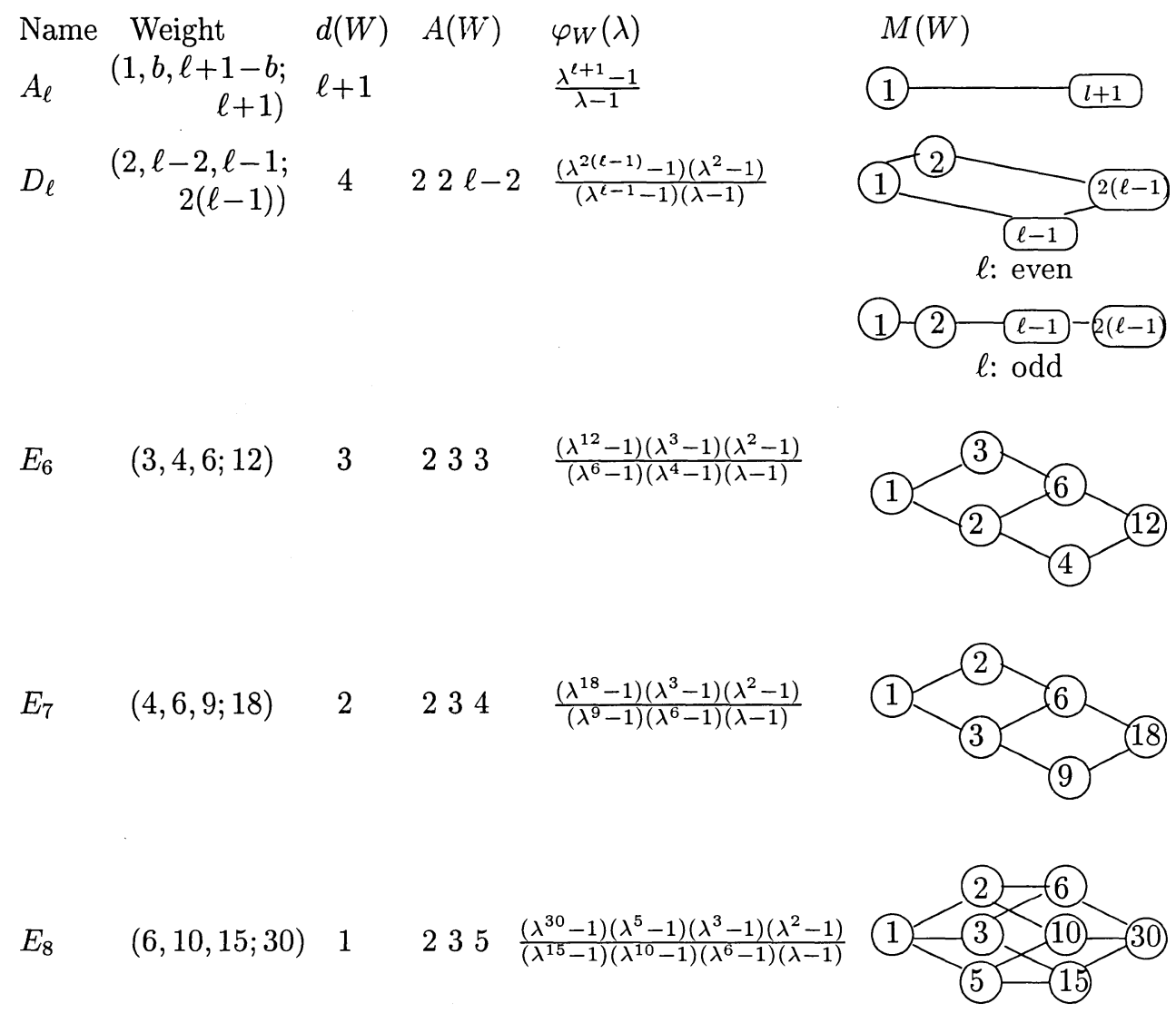

Converse to the observation iv), a simple weight system $W$ satisfies $\operatorname{Div}(h)=$ $M(W)$ if and only if $W$ is one of the following cases.

I. $\left(p_{2} p_{3}, p_{3} p_{1}, p_{1} p_{2} ; p_{1} p_{2} p_{3}\right)$ for three mutually different prime numbers $p_{1}, p_{2}$ and $p_{3} . \mu=\left(p_{1}-1\right)\left(p_{2}-1\right)\left(p_{3}-1\right)$.

II. $\left(p_{2}^{2}, p_{1} p_{2}, p_{1}\left(p_{2}-1\right) ; p_{1} p_{2}^{2}\right)$ for two different prime numbers $p_{1}$ and $p_{2} \cdot \mu=$ $\left(p_{1}-1\right)\left(p_{2}^{2}-p_{2}+1\right)$.

III. $\left(p_{2}, p_{1} q_{2}, p_{1} q_{3} ; p_{1} p_{2}\right)$ for two different prime numbers $p_{1}, p_{2}$ and two positive integers $q_{2}, q_{3}$ such that $p_{2}+1=\left(q_{2}+1\right)\left(q_{3}+1\right) . \mu=\left(p_{1}-1\right)\left(p_{2}+1\right)$.

IV. $\left(p^{2}, p(p-1), p^{2}-p+1 ; p^{3}\right)$ for a prime number $p . \mu=p^{3}-p^{2}+p-1$.

V. $(l m-m+1, m k-k+l, k l-l+1 ; p)$ for a prime number $p$ and three positive integers $k, l$ and $m$ such that $k l m=p-1 . \mu=p-1=k l m$.

$\mathrm{XI}$.

$(p, 2,(p-1) / 2 ; 2 p)$ for a prime number $p$ with $p \equiv 3 \bmod 4 . \mu=3 p+1$.

$(p, 3, p-1 ; 3 p)$ for a prime number $p$ with $p \equiv 2 \bmod 3 . \mu=4 p+2$.

Proof. Let $h=P_{1}^{E_{1}} \cdots P_{k}^{E_{k}}$ for prime numbers $P_{1}, \cdots, P_{k}$ and positive integers $E_{1}, \cdots, E_{k}$. A priori, we see $E_{i} \leq 3$, else the four elements $P_{i}, P_{i}^{2}, P_{i}^{3}$ and $P_{i}^{4}$ in $\operatorname{Div}(h)$ 
cannot be realized in $M(W)$. Clearly \#Div $(h)=\prod_{i=1}^{k}\left(E_{i}+1\right)$. On the other hand, the possible values of $\# M(W)$ are only $2,3,4,5,6$ or 8 . Thus $\operatorname{Div}(h)=M(W)$ implies a very limited possibility of $E_{i}$. Let us list possibilities of $p_{1}, p_{2}$ and $p_{3}$ according to the value $N:=\# M(W)$.

$N=2$. Then $k=1$ and $E_{1}=1$.

i) $p_{1}=P_{1}, p_{2}=P_{1}$ and $p_{3}=P_{1}$. Type $\mathrm{V}$.

$N=3$. Then $k=1$ and $E_{1}=2$.

ii) $p_{1}=P_{1}, p_{2}=P_{1}$ and $p_{3}=P_{1}^{2}$, Type XIV.

iii) $p_{1}=P_{1}, p_{2}=P_{1}^{2}$ and $p_{3}=P_{1}^{2}$, Type XIII.

These cases cannot be simple (Theorem 6.2).

$N=4$. iv) $k=2, E_{1}=E_{2}=1 . p_{1}=P_{1}, p_{2}=P_{2}$ and $p_{3}=P_{1} P_{2}$. Type XI.

v) $k=2, E_{1}=E_{2}=1 . p_{1}=P_{1}, p_{2}=p_{3}=P_{2}$, Type III,

vi) $k=1$ and $E_{1}=3$.

$N=6$. vii) $k=2$ and $E_{1}=1, E_{2}=2 . p_{2}=P_{1}, p_{2}=P_{2}$ and $p_{3}=P_{2}^{2}$. Type II.

$N=8 . k=2$ and $E_{1}=1, E_{2}=3$. This is impossible.

viii) $k=3$ and $E_{1}=E_{2}=E_{3}=1 . p_{1}=P_{1}, p_{2}=P_{2}$ and $p_{3}=P_{3}$. Type $\mathrm{I}$.

For a sake of Example 13.7, we list the 3 degenerate weight systems having only non-negative exponents. They correspond to simply elliptic singularities [S2]. So, let us call them elliptic weight systems. All elliptic weight systems have the same signature: $\left(a_{0} ; A(W)\right)=(1 ; \phi)$. The lattice of vanishing cycles for them are described by elliptic root systems [S41-S45].

Table C.

$$
\begin{aligned}
& \text { name } W \quad \mu d(W) \text { exponents } \quad \varphi_{W}(\lambda) \quad M(W) \\
& \widetilde{E}_{6} \quad(1,1,1 ; 3) \quad 8 \quad 27 \quad \begin{array}{rrr}
0,1,1,1,2, \\
2,2,3
\end{array} \frac{\left(\lambda^{3}-1\right)^{3}}{\lambda-1} \\
& \widetilde{E}_{7} \quad(1,1,2 ; 4) \quad 9 \quad 32 \quad \begin{array}{r}
0,1,1,2,2, \\
2,3,3,4
\end{array} \quad \frac{\left(\lambda^{4}-1\right)^{2}\left(\lambda^{2}-1\right)}{\lambda-1} \\
& \widetilde{E}_{8} \quad(1,2,3 ; 6) 10 \quad 36 \quad \begin{array}{r}
0,1,2,2,3, \\
3,4,4,5,6
\end{array} \quad \frac{\left(\lambda^{6}-1\right)\left(\lambda^{3}-1\right)\left(\lambda^{2}-1\right)}{\lambda-1}
\end{aligned}
$$
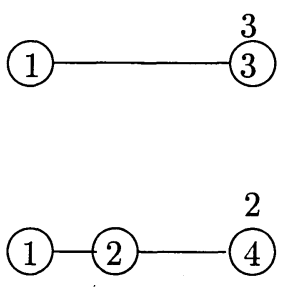

12. Strange duality of Arnold. We give an interpretation of the Arnold's strange duality by the duality of weight system. Still, the original duality remains somewhat mysterious. First, we review the strange duality in its original form.

In [A1], Arnold classified unimodular germs of holomorphic functions. They consist of three classes: i) three simple elliptic singularities $\widetilde{E}_{6}, \widetilde{E}_{7}, \widetilde{E}_{8}$, ii) cusp singulari- 
ties $T_{p q r}$ for $p, q, r \in \mathbb{N}$ with $\frac{1}{p}+\frac{1}{q}+\frac{1}{r}<1$, and iii) 14 exceptional unimodular singularities. In each of 1 parameter family of the 14 singularities, there is just one singularity which is defined by a weighted homogeneous polynomial $f(x, y, z)$ of 3 variables. The corresponding hypersurface $X_{0}$ (= the zero loci $\left\{(x, y, z) \in \mathbb{C}^{3}: f(x, y, z)=0\right\}$ of $f$ ) has the following description due to I.V. Dolgachev [D1-2]. The action of the Schwarz triangular group $\Gamma_{p q r}$ (with $1 / p+1 / q+1 / r<1$ ) on the upper half plane $\mathbb{H}$ lifts to a free action on the half-space $\widetilde{\mathbb{H}}:=\left\{(u, v) \in \mathbb{C}^{2}: \Im(u / v)>0\right\}$ so that $\widetilde{\mathbb{H}} / \Gamma$ is a complex 2-fold. By adding a cusp point $O, \widetilde{\mathbb{H}} / \Gamma \cup\{O\}$ becomes an affine algebraic variety with an isolated singularity at $O$. Dolgachev has shown that there are 14 triangular groups, for which $\mathbb{H} / \Gamma \cup\{O\}$ becomes a hypersurface in $\mathbb{C}^{3}$, and which are exactly the 14 quasi-homogeneous exceptional singularities $X_{0}$. The values $p, q$ and $r$ of the triangle are called the Dolgachev numbers.

On the other hand, Gabrielov [G] has determined the intersection form on the middle (=2-) dimensional homology group of a smoothing $X_{1}\left(=\left\{(x, y, z) \in \mathbb{C}^{3}\right.\right.$ | $f(x, y, z)=1\}$ for the 14 exceptional unimodular singularities (sometimes called the lattice of vanishing cycles for the singularity) as follows. We denote by $\tau_{p q r}$ the based lattice, whose intersection diagram is of the shape of the letter $T$ with three branches of length $p, q$ and $r$ (e.g. $E_{8}=\tau_{235}, E_{7}=\tau_{234}$, etc). Then the intersection form for the 14 exceptional unimodular singularities are of the form $\tau_{p q r} \oplus\left[\begin{array}{ll}0 & 1 \\ 1 & 0\end{array}\right]$, where the integers $p, q$ and $r$ are called the Gabrielov numbers. ${ }^{*}$ )

Then Arnold [A1] called a strange duality: there exists an involution $\sigma$ among the 14 exceptional singularities, by which the Dolgachev numbers and the Gabrielov numbers interchange. The Dolgachev and Gabrielov numbers are recalled in Table D. We include in the table the unique weight system $W$ attached to the singularity by the relation: $X_{0} \simeq X_{W}$ (for the uniqueness of $W$, see [S1, 4.3]).

Table D.

$\begin{array}{clllclll}\text { Name, } & \text { Weights, } & \text { D \#, } & \text { G \# } & \text { Name, } & \text { Weights, } & \text { D \#, G \# } \\ U_{12} & (3,4,4 ; 12) & 444 & 444 & W_{12} & (4,5,10 ; 20) & 255 & 255 \\ S_{12} & (3,4,5 ; 13) & 345 & 345 & Z_{13} & (3,5,9 ; 18) & 335 & 247 \\ S_{11} & (4,5,6 ; 16) & 256 & 344 & Z_{12} & (4,6,11 ; 22) & 246 & 246 \\ Q_{12} & (3,5,6 ; 15) & 336 & 336 & Z_{11} & (6,8,15 ; 30) & 238 & 245 \\ Q_{11} & (4,6,7 ; 18) & 247 & 335 & E_{14} & (3,8,12 ; 24) & 334 & 239 \\ Q_{10} & (6,8,9 ; 24) & 239 & 334 & E_{13} & (4,10,15 ; 30) & 245 & 238 \\ W_{13} & (3,4,8 ; 16) & 344 & 256 & E_{12} & (6,14,21 ; 42) & 237 & 237\end{array}$

REMARK 12.1. It was remarked by D. B. Fuks (cf. [A]) that the sum of the Dolgachev numbers and Gabrielov numbers for the 14 singularities are 24 for all 14 singularities. This fact was interpreted by Dolgacher, Nikulin and Pinkham in terms of duality between algebraic cycles and transcendental cycles on certain $K 3$ surfaces. This leads to further generalizations of the duality by several authors $[\mathrm{N}][\mathrm{P} 1][\mathrm{L}][\mathrm{E}-\mathrm{W}]$.

We now give an explanation of the strange duality in terms of weight system. The first step is the characterization of the 14 weight systems in Table D as follows.

ASSERTION 12.2. The weight systems attached to the 14 unimodular exceptional singularities are exactly the simple and non-degenerate weight systems having -1 as its smallest exponent.

This is a matter of calculation. Table $\mathrm{E}$ gives the list of such weight systems together with the numerical data including the characteristic functions and the posets. The "*" means that the weight system is self-dual. 
Table E.

Weight $\quad \mu_{W} \quad d(W) \quad A(W) \quad \varphi_{W}(\lambda) \quad M(W)$

$*(3,4,4 ; 12) \quad 12 \quad 16 \quad 444 \quad \frac{\left(\lambda^{12}-1\right)\left(\lambda^{4}-1\right)}{\left(\lambda^{3}-1\right)(\lambda-1)}$

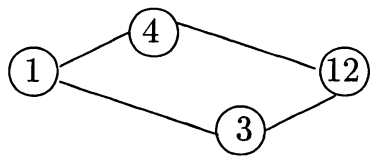

$*(3,4,5 ; 13) \quad 12 \quad 13 \quad 345 \quad \frac{\left(\lambda^{13}-1\right)}{(\lambda-1)}$

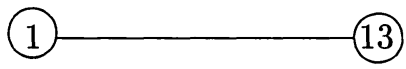

$(4,5,6 ; 16) \quad 11 \quad 8 \quad 256 \quad \frac{\left(\lambda^{16}-1\right)\left(\lambda^{4}-1\right)}{\left(\lambda^{8}-1\right)(\lambda-1)}$

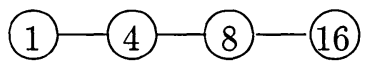

$*(3,5,6 ; 15) \quad 12 \quad 9 \quad 336 \quad \frac{\left(\lambda^{15}-1\right)\left(\lambda^{3}-1\right)}{\left(\lambda^{5}-1\right)(\lambda-1)}$

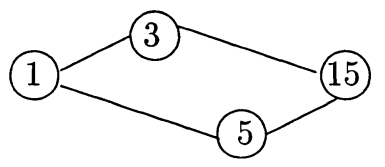

$(4,6,7 ; 18) \quad 11 \quad 6 \quad 247 \quad \frac{\left(\lambda^{18}-1\right)\left(\lambda^{3}-1\right)}{\left(\lambda^{9}-1\right)(\lambda-1)}$

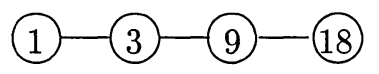

$(6,8,9 ; 24) \quad 10 \quad 3 \quad 239 \quad \frac{\left(\lambda^{24}-1\right)\left(\lambda^{4}-1\right)\left(\lambda^{3}-1\right)}{\left(\lambda^{12}-1\right)\left(\lambda^{8}-1\right)(\lambda-1)}$

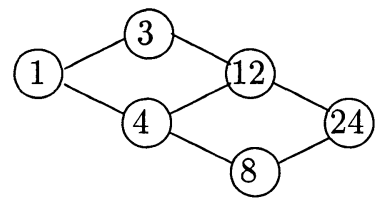

$(3,4,8 ; 16) \quad 13 \quad 8 \quad 344 \quad \frac{\left(\lambda^{16}-1\right)\left(\lambda^{2}-1\right)}{\left(\lambda^{4}-1\right)(\lambda-1)}$

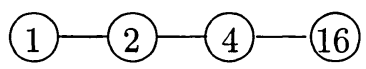

$*(4,5,10,20) \quad 12 \quad 5 \quad 255 \quad \frac{\left(\lambda^{20}-1\right)\left(\lambda^{5}-1\right)\left(\lambda^{2}-1\right)}{\left(\lambda^{10}-1\right)\left(\lambda^{4}-1\right)(\lambda-1)}$<smiles>C1=CC2=CC=C3C=Cc4ccc(cc4C=C3C=C2)C=C1</smiles>

$(3,5,9 ; 18) \quad 13 \quad 6 \quad 335 \quad \frac{\left(\lambda^{18}-1\right)\left(\lambda^{2}-1\right)}{\left(\lambda^{6}-1\right)(\lambda-1)}$

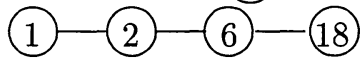

$*(4,6,11 ; 22) \quad 12 \quad 4 \quad 246 \quad \frac{\left(\lambda^{22}-1\right)\left(\lambda^{2}-1\right)}{\left(\lambda^{11}-1\right)(\lambda-1)}$

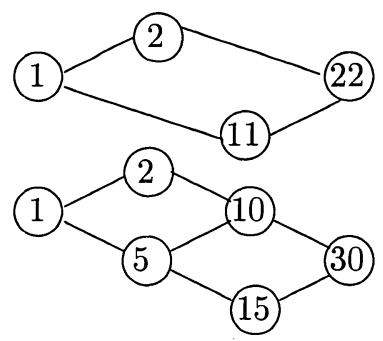

$(6,8,15 ; 30) \quad 11 \quad 2 \quad 238 \quad \frac{\left(\lambda^{30}-1\right)\left(\lambda^{5}-1\right)\left(\lambda^{2}-1\right)}{\left(\lambda^{15}-1\right)\left(\lambda^{10}-1\right)(\lambda-1)}$ 


$$
\begin{array}{llllll}
(3,8,12 ; 24) & 14 & 3 & 334 & \frac{\left(\lambda^{24}-1\right)\left(\lambda^{3}-1\right)\left(\lambda^{2}-1\right)}{\left(\lambda^{8}-1\right)\left(\lambda^{6}-1\right)(\lambda-1)} \\
*(6,10,15 ; 30) & 13 & 2 & 245 & \frac{\left(\lambda^{30}-1\right)\left(\lambda^{3}-1\right)\left(\lambda^{2}-1\right)}{\left(\lambda^{15}-1\right)\left(\lambda^{6}-1\right)(\lambda-1)}
\end{array}
$$

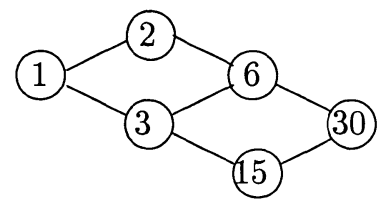

It was shown that the set \{simple and non-degenerate weight system having -1 as its smallest exponent\} is closed under the involution $W \leftrightarrow W^{*}$ (Theorem 7.102 ). Comparing Tables D and E, we obtain:

Assertion 12.3.

2. The dual pairs $W \leftrightarrow W^{*}$ of the weight systems in Theorem 7.102 coincide with the dual pairs of strange duality of Arnold.

3. For the 6 self-dual weight systems, one has the equality $\operatorname{Div}(h) \equiv M(W)$. It is a natural question whether the duality of weight system explains the strange duality. To answer the question, we first notice a fact that the Dolgachev \# is elementarily determined by the weight system $W$.

Assertion 12.4.

4. The set of Dolgachev numbers is equivalent to the signature $A(W)$ defined for the weight system $W$ in (10.1).

Proof. This may almost be a triviality now, when we identify the surface $\mathbb{H} / \Gamma$ considered by Dolgachev and the hypersurface $X_{W} \backslash\{O\}$ in $\mathbb{C}^{3}$ defined by the weight system $W$ (cf. Remark 10.3 at the end of $\S 10$ ), where the isotropy groups of $\mathbb{C}^{\times}$-actions on them has two different expressions: one by the conjugacy classes of isotropy groups of the action of $\Gamma$ on $\mathbb{H}$, and the other by the singular orbits of $\mathbb{C}^{\times}$-action on $X_{W} \backslash\{O\}$ given by the points $C_{W} \cap\left(\ell_{x} \cup \ell_{y} \cup \ell_{z}\right)(\simeq A(W))$.

The following diagram symbolized the relations among the data we study.

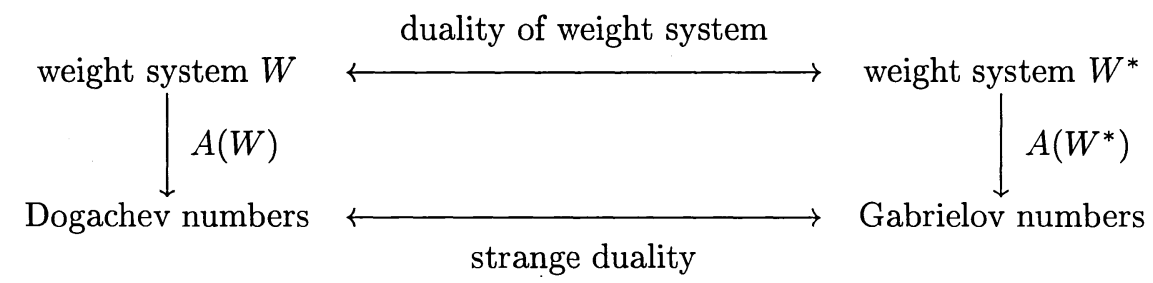

Here the left vertical arrow is explained by Assertion 12.4 and the first horizontal arrow is explained in Theorem $7.102(\S 7)$. The right vertical arrow remains to be 
explained yet, which is proven by Gabrielov [Ga] depending on the classification of the 14 unimodular exceptional singularities as formulated in the next assertion.

ASSERTION 12.5.

5. Let $W$ be a simple weight system having -1 as its smallest exponent. Then the lattice of the vanishing cycles for $W$ is isomorphic to $\tau_{A\left(W^{*}\right)} \oplus\left[\begin{array}{ll}0 & 1 \\ 1 & 0\end{array}\right]$.

So, if one can give a proof of the Assertion 12.5 directly without a use of classification, then it completes the explanation of the strange duality. We ask the following some more a general problem:

For a weight system $W$, describe in terms of $W$ the lattice of vanishing cycles together with the intersection form and the Milnor monodromy action $c$ on it.

REMARK 12.6.

1. Converse of the above problem: the data of the monodromy $c$ determine $M(W)$ is true, since the characteristic polynomial $\varphi(\lambda):=\operatorname{det}(\lambda I-c)$ determines $M(W)$ (Theorem 5.1). Furthermore, according to Theorem 6.3, the datum of $M(W)$ determines $W$ up to finite ambiguities.

2. Experiences suggest that the Dolgachev numbers have close relations with $\iota(M(W))$, whereas the Gavrielov numbers have similarity with $M(W)$. Can one clarify this as a mathematical statement?

13. Eta products for a regular system of weights. Let $W$ be a regular system of weights. Inspired by finite group theory ([C-N], $[\mathrm{Ma}],[\mathrm{Ko}]$ ), we introduce products $\eta_{W}$ and $\eta_{W}^{*}$ of Dedekind eta-functions. They are modular forms on the upper half plane $\mathcal{H}$ of weight $a_{0}$ and $-a_{0}$, respectively, which may have poles at cusps. The products $\eta_{W}$ and $\left(\eta_{W}^{*}\right)^{-1}$ are holomorphic (resp. vanishing) at all cusps if and only if the dual rank $\nu_{W}$ is non-positive (rep. negative). The level and character of the etaproducts are explicitly given in Lemma 13.3. The products $\eta_{W}$ and $\eta_{W}^{*}$ are combined by the involution $\tau \rightarrow-1 / h \tau$ (13.4). This leads to an interpretation of the duality of weight systems in terms of $\eta$-products (13.5) and (13.5)*.

Let $W$ be a weight system, and let $e_{W}$ be the cyclotomic exponent (3.1) of its characteristic polynomial $\varphi_{W}(2.1)$. Define the products:

$$
\begin{aligned}
\eta_{W}(\tau) & :=\prod_{i \in M(W)} \eta(i \tau)^{e_{W}(i)}, \\
\eta_{W}^{*}(\tau) & :=\prod_{j \mid h} \eta(j \tau)^{-e_{W}(h / j)} .
\end{aligned}
$$

Here $\eta(\tau):=q^{1 / 24} \prod_{n=1}^{\infty}\left(1-q^{n}\right)$ with $q=\exp (2 \pi \sqrt{-1} \tau)$ is the Dedekind eta-function. We call $\eta_{W}$ and $\eta_{W}^{*}$ the eta-products for $W$. The product expansions of $\eta$ is absolutely convergent for $q \in \mathbb{C}$ with $|q|<1$, so $\eta_{W}$ and $\eta_{W}^{*}$ are nowhere vanishing holomorphic functions in $\tau \in \mathcal{H}:=\{\tau \in \mathbb{C}: \Im(\tau)>0\}$. Since the order of the product in $\eta_{W}$ and $\eta_{W}^{*}$ can be changed freely, one has some multiplicative expressions for the eta-products:

$$
\begin{aligned}
\eta_{W}(\tau) & =q^{\mu_{W} / 24} \prod_{i \in M(W)} \prod_{n=1}^{\infty}\left(1-q^{i n}\right)^{e_{W}(i)} \\
& =q^{\mu_{W} / 24} \prod_{n=1}^{\infty} \varphi_{W}\left(q^{n}\right)
\end{aligned}
$$




$$
=q^{\mu_{W} / 24} \prod_{n=1}^{\infty} \prod_{i=1}^{\mu_{W}}\left(q^{n}-\exp \left(2 \pi \sqrt{-1} m_{i} / h\right)\right)
$$

$$
\begin{aligned}
\eta_{W}^{*}(\tau) & =q^{\nu_{W} / 24} \prod_{j \mid h} \prod_{n=1}^{\infty}\left(1-q^{j n}\right)^{-e_{W}(h / j)} \\
& =q^{\nu_{W} / 24} \prod_{n=1}^{\infty} \varphi_{W}^{*}\left(q^{n}\right) \\
& =q^{\nu_{W} / 24} \prod_{n=1}^{\infty}\left(\prod_{i=1}^{\nu_{W}+2 a_{0}}\left(q^{n}-\exp \left(2 \pi \sqrt{-1} m_{i}^{*} / h\right)\right) /\left(q^{n}-1\right)^{2 a_{0}}\right)
\end{aligned}
$$

where $m_{1}, \ldots, m_{\mu}\left(\mu=\mu_{W}\right)$ and $m_{1}^{*}, \ldots, m_{\nu+2 a_{0}}^{*}\left(\nu=\nu_{W}\right)$ are the exponents and dual exponents for $W$, respectively (cf. (2.2) and (7.5)). Let us give a relation between the two eta-products.

ASSERTION 13.1. The two eta-products $\eta_{W}$ and $\eta_{W}^{*}$ are combined by the imaginary transformation: $\tau \rightarrow-1 / h \tau$, as follows.

$$
\begin{gathered}
\eta_{W}(-1 / h \tau) \cdot \eta_{W}^{*}(\tau)=(\tau / \sqrt{-1})^{a_{0}} / \sqrt{d^{*}(W)} . \\
\eta_{W}(\tau) \cdot \eta_{W}^{*}(-/ h \tau)=(\sqrt{-1} / \tau)^{a_{0}} / \sqrt{d(W)} .
\end{gathered}
$$

where $h=h_{W}$ is the Coxeter number $(\S 1)$ and $d(W)$ (resp. $d^{*}(W)$ ) are the (resp. dual) discriminant $((9.1),(9.2))$ for the weight system $W$.

Proof. The transformation $\tau \rightarrow-1 / h \tau$ is involutive. Hence, in view of (9.3), $(13.3)^{*}$ is a consequence of (13.3). So we prove only (13.3).

By a use of the well-known formula $\eta(-1 / \tau)=\sqrt{\tau / \sqrt{-1}} \eta(\tau)$ for the Dedekind-eta function, the first factor of (13.3) is calculated as:

$$
\begin{aligned}
\eta_{W}(-1 / h \tau) & =\prod_{i \in M(W)} \eta(-1 /(h \tau / i))^{e_{W}(i)} \\
& =\prod_{i \in M(W)}(\sqrt{h \tau / i \sqrt{-1}})^{e_{W}(i)} \cdot \eta(h \tau / i)^{e_{W}(i)} \\
& =\sqrt{d}^{-1} \cdot(h \tau / \sqrt{-1})^{a_{0}} \cdot \prod_{j i h} \eta(j \tau)^{e_{W}(h / j)} \\
& =\sqrt{d}^{-1} \cdot(h \tau / \sqrt{-1})^{a_{0}} \cdot \eta_{\mathrm{W}}^{\mathbf{x}} \cdot(\tau)^{-1}
\end{aligned}
$$

Suppose $W$ and $W^{*}$ are weight systems which are dual to each other ( $\left.\S 7\right)$. Then by definition of the eta-products, one has

$$
\eta_{W}(\tau)=\eta_{W^{*}}^{*}(\tau) \text { and } \eta_{W}^{*} \cdot(\tau)=\eta_{W^{*}}(\tau) .
$$

Combining (13.4) with (13.3) and (13.3)*, we obtain another expression of the duality of weight system in terms of eta-products.

Corollary 13.2. Let $W$ and $W^{*}$ be dual weight systems. Then,

$$
\eta_{W}(-1 / h \tau) \cdot \eta_{W^{*}}(\tau)=\sqrt{d}^{-1}
$$


Here $d:=d(W)=d\left(W^{*}\right)$. Conversely, the (13.5) characterize the duality between $W$ and $W^{*}$ so far their poset is not self-dual.

Proof. We have only to recall the fact: $\sum_{i} e_{W}(i)=2 a_{0}=0$, since $W$ is non degenerate (cf. (7.5) and $\S 7$ Assertion $7.71 \mathrm{i}$ )). The (13.5) is equivalent to (7.7).

Next, we discuss the automorphic nature of the eta-products. For a function $f(\tau)$ on the upper half plane $\mathcal{H}$ and for $A=\left(\begin{array}{ll}a & b \\ c & d\end{array}\right) \in G L_{2}^{+}(\mathbb{R})$ and $k \in \mathbb{Z}$, put $\left.f\right|_{k} A:=$ $\operatorname{det}(A)^{k / 2}(c \tau+d)^{-k} f(A(\tau))$ with $A(\tau):=(a \tau+b) /(c \tau+d)$. If $f$ is holomorphic on $\mathcal{H}$ and there exist $k, N \in \mathbb{Z}_{>0}$ such that $\left.f\right|_{k} A=\varepsilon(d) f$ for $A \in \Gamma_{0}(N):=\{A=$ $\left.\left(\begin{array}{ll}a & b \\ c & d\end{array}\right) \in S L_{2}(\mathbb{Z}): c \equiv 0 \bmod N\right\}$ where $\varepsilon$ is a Dirichlet character $\bmod N$, then we call $f$ an almost holomorphic automorphic form of weight $k$ and character $\varepsilon$ (or, of type $(k, \varepsilon)$ ) on the group $\Gamma_{0}(N)$ (of level $N$ ). The eta-products $\eta_{W}$ and $1 / \eta_{W^{*}}$ are almost automorphic forms of weight $a_{0}$, since one has $\left.\eta\right|_{1 / 2} A=V(A) \cdot \eta$ for ${ }^{\forall} A \in S L_{2}(\mathbb{Z})$ where $V(A)$ is a 24th root of unity whose explicit formula is known (cf. [Ra, p163]). An explicit description of level $N$ and character $\varepsilon$ of the eta-products are given in the next lemma (cf. [Sa12 §2 Lemma 1]).

LEMMA 13.3. The eta-products $\eta_{W}\left(m_{W} \tau\right)$ and $1 / \eta_{W}^{*}\left(m_{W}^{*} \tau\right)$ are almost holomorphic automorphic forms of weight $a_{0}$ and character $\varepsilon_{W}$ on the group $\Gamma_{0}\left(N_{W}\right)$, where $m_{W}, m_{W}^{*}, \varepsilon_{W}$ and $N_{W}$ are given as follows.

$$
\begin{gathered}
m_{W}:=24 /\left(\operatorname{gcd}\left(\mu_{W}, 24\right), \quad m_{W}^{*}:=24 / \operatorname{gcd}\left(\nu_{W}, 24\right)\right. \\
N_{W}:=h_{W} m_{W} m_{W}^{*} \\
\varepsilon_{W}(d):= \begin{cases}\left(\frac{d_{s f}(-1)^{a_{0}}}{d}\right) & \text { for } d \text { odd } \\
\left(\frac{d}{d_{s f}}\right) & \text { for d even }\end{cases}
\end{gathered}
$$

Here $d_{s f}$ is the square free part of the discriminant (9.1) and $\left(\frac{c}{d}\right)$ is the residue symbol defined as follows. If $d>0$ and odd, it is the Legendre symbol multiplicatively extended in $d$. If $d<0$ then $\left(\frac{c}{d}\right)=\left(\frac{c}{|d|}\right)$ for $d<0$ and $c>0$ and $\left(\frac{c}{d}\right)=-\left(\frac{c}{|d|}\right)$ for $d<0$ and $c<0$. Put $\left(\frac{0}{ \pm 1}\right):=1$.

The next lemma is the goal of this section.

LEMMA 13.4. The eta-products $\eta_{W}(m \tau)$ and $1 / \eta_{W}^{*}\left(m^{*} \tau\right)$ are holomorphic (resp. have zero) at all cusp points $\in \mathbb{Q} \cup\{\infty\}$, if and only if $\nu(W) \leq 0$ (resp. $<0)$.

Proof. The following fact may be well known among experts. For a sake of completeness, we recall with a sketch of proof (cf. [H-M, Theorem 1]).

Fact. For all $A=\left(\begin{array}{ll}a & b \\ c & d\end{array}\right) \in S L_{2}(\mathbb{Z})(c \neq 0),\left.\eta_{W}(m \tau)\right|_{a_{0}} A$ can be developed in a Laurent series in the local parameter $\exp \left(2 \pi \sqrt{-1} \tau / 24 m h_{W}\right)$ whose leading degree is given by $c \cdot \Phi_{W}(c)$. Here for any $\xi \in \mathbb{Z}_{\neq 0}$, put

$$
\xi \cdot \Phi_{W}(\xi):=\sum_{i \in M(W)}\left(h \cdot(i, \xi)^{2} / i\right) e_{W}(i)
$$

Sketch of a proof of the Fact. 
Put $f(\tau):=\eta_{W}(m \tau)$, where $m:=m_{W}$. Let $\frac{a}{c}=A(\sqrt{-1} \infty)$ for $A=\left(\begin{array}{ll}a & b \\ c & d\end{array}\right) \epsilon$ $S L_{2}(\mathbb{Z})$ and $c \neq 0$ be a cusp. For $i \in M(W)$, let $\left(\begin{array}{cc}\alpha_{i} & \beta_{i} \\ 0 & \delta_{i}\end{array}\right) \in S L_{2}(\mathbb{Z})\left(\begin{array}{cc}m i & 0 \\ 0 & 1\end{array}\right) A$, i.e. ${ }^{\exists} B_{i} \in S L_{2}(\mathbb{Z})$ s.t. $\left(\begin{array}{cc}m i & 0 \\ 0 & 1\end{array}\right) A=B_{i}\left(\begin{array}{cc}\alpha_{i} & \beta_{i} \\ 0 & \delta_{i}\end{array}\right)$.

Comparing the determinant and the gcd of the first column of both hand sides, one gets $m i=\alpha_{i} \delta_{i}$ and $(m i, c)= \pm \alpha_{i}$, respectively. Then

$$
\begin{aligned}
\left.f(\tau)\right|_{a_{0}} A & =\prod_{i \in M(W)}\left(\left.\eta(m i \tau)\right|_{1 / 2} A\right)^{e_{W}(i)} \\
& =\prod_{i \in M(W)}\left(\left.(m i)^{-1 / 4} \cdot \eta(\tau)\right|_{1 / 2}\left(\begin{array}{cc}
m i & 0 \\
0 & 1
\end{array}\right) A\right)^{e_{W}(i)} \\
& =C_{1} \cdot \prod_{i \in M(W)}\left(\left.\eta(\tau)\right|_{1 / 2} B_{i}\left(\begin{array}{cc}
\alpha_{i} & \beta_{i} \\
0 & \delta_{i}
\end{array}\right)\right)^{e_{W}(i)} \\
& =C_{2} \cdot \prod_{i \in M(W)}\left(\left.\eta(\tau)\right|_{1 / 2}\left(\begin{array}{cc}
\alpha_{i} & \beta_{i} \\
0 & \delta_{i}
\end{array}\right)\right)^{e_{W}(i)} \\
& =C_{3} \cdot \prod_{i \in M(W)}\left(\eta\left(\frac{\alpha_{i}}{\delta_{i}} \tau+\frac{\beta_{i}}{\delta_{i}}\right)\right)^{e_{W}(i)} \\
& =C_{4} \cdot \exp \left(2 \pi \sqrt{-1}\left(\sum_{i \in M(W)} \frac{\alpha_{i}}{\delta_{i}} e(i)\right) \tau / 24\right) \cdot \prod_{i \in M(W)} F_{i}(\tau)
\end{aligned}
$$

where $C_{1}, C_{2}, C_{3}$ and $C_{4}$ are non-zero constants and $F_{i}(\tau)$ is the unit factor of $\left(\eta\left(\frac{\alpha_{i}}{\delta_{i}} \tau+\frac{\beta_{i}}{\delta_{i}}\right)\right)^{e_{W}(i)}$, which can be developed into a power series in $q_{i}:=\exp \left(\frac{2 \pi \sqrt{-1} \tau}{\delta_{i}}\right)$ $(i \in M(W))$ with constant term 1 . Since $\delta_{i}|m i| m \cdot h_{W}$, all variables are positive powers of $\exp \left(2 \pi \sqrt{-1} \tau / 24 m h_{W}\right)$ and $\prod F_{i}$ is expanded in its power series with a constant term 1 . The degree of the leading term of $\left.f(\tau)\right|_{a_{0}} A$ w.r.t. the variable is given by

$$
\begin{aligned}
\left(\sum_{i \in M(W)}\left(\alpha_{i} / \delta_{i}\right) e_{W}(i)\right) \cdot m h_{W} & =\left(\sum_{i \in M(W)}\left(\alpha_{i}^{2} / m i\right) e_{W}(i)\right) \cdot m h_{W} \\
& =\sum_{i \in M(W)} h_{W}(i, c)^{2} e_{W}(i) / i=c \cdot \Phi_{W}(c) .
\end{aligned}
$$

We return to the proof of Lemma 13.4 .

Since $\Phi_{W}(1)=-\nu_{W}(7.2)$ (that is: the order of pole of $\eta_{W}$ at an integral cuspidal point is equal to $\nu_{W}$ ), it is necessary to be $\nu_{W} \leq 0$ (resp. $\left.<0\right)$ for $\eta_{W}$ to be holomorphic (cuspidal). To prove the converse, it is sufficient to prove the innequality:

$$
\xi \cdot \Phi_{W}(\xi) \geq \xi^{2} \cdot \Phi_{W}(1)
$$

for any $\xi \in \mathbb{Z}_{\neq 0}$. By definition of $\Phi_{W}$, one has:

$$
\xi \cdot \Phi_{W}(\xi)-\xi^{2} \cdot \Phi_{W}(1)=\sum_{i \in M(W)}\left(h \cdot(i, \xi)^{2} / i-\xi^{2} \cdot(h / i)\right) e_{W}(i) .
$$


We decompose the sum according to the level $n(i)=0,1,2$ or 3 . The term for $n(i)=3$ (i.e. $i=h$ ) is cancelled automatically. So, the sum is equal to

$$
\begin{aligned}
& \xi^{2} \cdot h-h-\sum_{n(i)=1}\left(\xi^{2} \cdot(h / i)-h \cdot(i, \xi)^{2} / i\right) \\
& +\sum_{n(i)=2}\left(\xi^{2} \cdot(h / i)-h \cdot(i, \xi)^{2} / i\right)\left|e_{W}(i)\right| \\
& =h \cdot\left(\xi^{2}-1\right)\left(1-\sum_{n(i)=1} 1 / i\right) \\
& +h \cdot \sum_{n(i)=1}\left((i, \xi)^{2}-1\right) / i \\
& +\sum_{n(i)=2}\left(\xi^{2}-(i, \xi)^{2}\right)\left|e_{W}(i)\right| \cdot(h / i) .
\end{aligned}
$$

The second and the third terms in the last expression are non-negative. The index set $I:=\{i \in M(W) \mid n(i)=1\}$ consists at most of three integers, which are non less than 2 and mutually different (cf. (4.4)). So, the factor $1-\sum_{n(i)=1} 1 / i$ of the first term can be negative only when $I=\{2,3,5\}$ and $W$ is of type $E_{8}$. The explicit formula of $\xi \cdot \Phi_{W}(\xi)$ using $e_{W}(i)=(-1)^{n(i)}$ for $i \in M\left(E_{8}\right)=D(30)$ proves (13.10) for this case.

Altogether, the inequality (13.10) is proven.

These complete a proof of the lemma.

In the rest of section, apart from duality, we ask a question on non-negativity of Fourier coefficients of eta-products. The following is the conjecture.

Conjecture 13.5. Let $W$ be a weight system. All Fourier coefficients at $\infty$ of $\eta\left(h_{W} \cdot \tau\right)^{\nu} \cdot \eta_{W}(\tau)$ are non-negative integers, if and only if $\nu_{W} \geq \nu$. All Fourier coefficients at $\infty$ of $\eta\left(h_{W} \cdot \tau\right)^{\mu} \cdot \eta_{W}^{*}(\tau)$ are non-negative integers, if and only if $\mu_{W} \geq \mu$.

Specialization of the conjecture to $\nu=0$ is the one given in the introduction. Specialization of the conjecture to $\mu=0$ is the following fact.

Fact. Fourier coefficients at $\infty$ of $\eta_{W}^{*}(\tau)$ are non-negative integers.

This fact is a direct consequence of a product formula of the eta products given below. Note that the formula says also that $\eta_{W}$ has non-negative Fourier coefficients at $\infty$ if $W$ is non-degenerate. Since $\nu_{W}+2 a_{0}>0$, this is a particular case of the conjecture. It implies that the eta-product for a weight system having its dual has non-negative Fourier coefficients at $\infty$.

ASSERTION 13.6. Let $\bar{m}_{1}, \ldots, \bar{m}_{\mu}$ and $m_{1}^{*}, \ldots, m_{\nu+2 a_{0}}^{*}$ be the sets of reduced $\left.{ }^{*}\right)$ exponents and dual exponents (Def. 7.3) for $W$, respectively. Then,

$$
\begin{aligned}
& \eta_{W}(\tau)=q^{\mu(\mu) / 24} \prod_{n=0}^{\infty}\left(\left(1-q^{n h}\right)^{2 a_{0}} / \prod_{i=1}^{\nu+2 a_{0}}\left(1-q^{m_{i}^{*}+n h}\right)\right) . \\
& \eta_{W}^{*}(\tau)=q^{\nu(\mu) / 24} \prod_{n=0}^{\infty}\left(1 / \prod_{i=1}^{\mu}\left(1-q^{\bar{m}_{i}+n h}\right)\right) .
\end{aligned}
$$

*) Here a reduced exponent $\bar{m}_{i}$ is an integer $0<\bar{m}_{i} \leq h$, which represents an exponent $m_{i} \bmod (h)$. 
Proof. We prove only (13.11). The proof of $(13.11)^{*}$ is proceeded similarly. The multiplicity $n(j)$ of a factor $\left(1-q^{j}\right)$ for $j \in \mathbb{Z}_{>0}$ in (13.2) is given by: $n(j):=$ $\sum_{i \in M(W), i \mid j} e_{W}(i)$, which clearly depends only on $\operatorname{gcd}(j, h)$. Recall the definition (7.1) for $\varphi_{W}^{*}(\lambda)$, and the fact that $n(j)$ is equal to minus of the multiplicity of the $h / \operatorname{gcd}(j, h)$ th primitive roots of unity in the cyclotomic equation $\varphi_{W}^{*}(\lambda)=0$ (cf. (7.4) and Assertion 7.2). So the factors for $j$ with $(n-1) h<j<n h$ and $n \in \mathbb{N}$ are given by

$$
\prod_{j=(n-1) h+1}^{n h-1}\left(1-q^{j}\right)^{n(j)}=1 / \prod_{i=1}^{\nu}\left(1-q^{n h-m_{i}^{*}}\right)
$$

where we use a fact $\left\{m_{1}^{*}, \ldots, m_{\nu}^{*}\right\}=\left\{h-m_{1}^{*}, \ldots, h-m_{\nu}^{*}\right\}$. If $\operatorname{gcd}(j, h)=h$, then,

$$
n(j)=n(h)=\sum_{i \in M(W)} e_{W}(i)=2 a_{0}
$$

These imply (13.11).

EXAMPLE 13.7. We illustrate the non-negativity of Fourier coefficients of $\eta_{W}$ for the weight systems of types $\widetilde{E}_{6}, \widetilde{E}_{7}, \widetilde{E}_{8}$ and $(1,1,2 ; 5)$.

Let $W$ be a weight system of type $\widetilde{E}_{6}, \widetilde{E}_{7}, \widetilde{E}_{8}$ or $(1,1,2 ; 5)$. Since $\nu_{W}=0$, the eta-product $\eta_{W}(\tau)$ is a holomorphic automorphic form of weight $a_{0}$ equal to 1 or 2 , respectively (Lemma 13.3). Let $L_{W}(s)=\sum_{n=1}^{\infty} c_{W}(n) n^{-s}$ be the Dirichlet series attached to the Fourier expansion $\eta_{W}(m \tau)=\sum c_{W}(n) q^{n}$. We shall determine "Euler product expression" of the Dirichlet series. This leads to an explicit formula for the Fourier coefficients $c_{W}(n)$ and the non-negativity of them. Results are exhibited in the following list. The Fourier expansion of the eta-product for the weight system $(1,1,2 ; 5)$ was studied by Ramanujan (who called the formula very strange). The expression of some eta-products including $\widetilde{E}_{6}$ by a use of Eisenstein series was studied by Koike in connection with moonshine [Ko].

$$
\begin{aligned}
& \widetilde{E}_{6}: W=(1,1,1 ; 3) \\
& \eta_{\widetilde{E}_{6}}(3 \tau)=\eta(9 \tau)^{3} / \eta(3 \tau) \\
& =q+q^{4}+2 q^{7}+2 q^{13}+2 q^{19}+q^{25}+2 q^{28}+\cdots \\
& L_{\widetilde{E}_{6}}(s)=\prod_{p \neq 3} \frac{1}{\left(1-\left(\frac{p}{3}\right) p^{-s}\right)\left(1-p^{-s}\right)} \\
& =\prod_{p \equiv 1(3)} \frac{1}{\left(1-p^{-s}\right)^{2}} \prod_{p \equiv 2(3)} \frac{1}{1-p^{-2 s}} \\
& c_{\widetilde{E}_{6}}(N)=\left\{\begin{array}{l}
0 \quad \text { if } n>0, \\
\left(n_{1}+1\right) \cdots\left(n_{k}+1\right)\left(1+(-1)^{m_{1}}\right) \cdots\left(1+(-1)^{m_{l}}\right) / 2^{l} \text { otherwise },
\end{array}\right.
\end{aligned}
$$

for $N=3^{n} p_{1}^{n_{1}} \cdots p_{k}^{n_{k}} q_{1}^{m_{1}} \cdots q_{l}^{m_{l}}$ where $p_{i}$ is a prime number with $p_{i} \equiv 1(3)$ and $q_{i}$ is a prime number with $q_{i} \equiv 2(3)$. 


$$
\begin{gathered}
\tilde{E}_{7}: W=(1,1,2 ; 4) \\
\eta_{\widetilde{E}_{7}(8 \tau \neq} \eta(32 \tau)^{2} \eta(16 \tau) / \eta(8 \tau) \\
=q^{3}+q^{11}+q^{19}+2 q^{27}+q^{43}+2 q^{51}+q^{59}+q^{67}+q^{75}+\cdots \\
L_{\widetilde{E}_{7}}(s)=\prod_{p \equiv 1(8)} \frac{1}{\left(1-p^{-s}\right)^{2}} \prod_{p \equiv 5,7(8)} \frac{1}{1-p^{-2 s}} \\
\quad \times\left(\prod_{p \equiv 3(8)} \frac{1}{\left(1-p^{-s}\right)^{2}}-\prod_{p \equiv 3(8)} \frac{1}{\left(1+p^{-s}\right)^{2}}\right) / 4 \\
c_{\widetilde{E}_{7}}\left(N \neq\left\{\begin{array}{c}
0 \quad \text { if } n>0, \\
1 \\
4 \prod_{i=1}^{k}\left(n_{i}+1\right)\left(1-(-1)^{\sum_{i=1}^{k} n_{i}}\right) \prod_{i=1}^{l}\left(m_{i}+1\right) \\
\prod_{i=1}^{t}\left(1+(-1)^{s_{i}}\right) / 2^{t} \text { otherwise },
\end{array}\right.\right.
\end{gathered}
$$

for $N=2^{n} p_{1}^{n_{1}} \cdots p_{k}^{n_{k}} q_{1}^{m_{l}} \cdots q_{l}^{m_{l}} r_{1}^{s_{1}} \cdots r_{t}^{s_{t}}$ where $p_{i}$ is a prime number with $p_{i} \equiv 3$ (8), $q_{i}$ is a prime number with $q_{i} \equiv 1$ (8) and $r_{i}$ is a prime number with $r_{i} \equiv 5$ or 7 (8).

$$
\begin{aligned}
& \widetilde{E}_{8}: W=(1,2,3 ; 6) \\
& \eta_{\widetilde{E}_{8}}(12 \tau)=\eta(72 \tau) \eta(36 \tau) \eta(24 \tau) / \eta(12 \tau) \\
& =q^{5}+q^{17}+q^{29}+q^{41}+q^{53}+2 q^{65}+q^{89}+q^{101}+q^{113}+\cdots \\
& L_{\widetilde{E}_{8}}(s)=\prod_{p \equiv 1(12)} \frac{1}{\left(1-p^{-s}\right)^{2}} \prod_{p \equiv 7,11(12)} \frac{1}{1-p^{-2 s}} \\
& \times\left(\prod_{p \equiv 5(12)} \frac{1}{\left(1-p^{-s}\right)^{2}}-\prod_{p \equiv 5(12)} \frac{1}{\left(1+p^{-s}\right)^{2}}\right) / 4 \\
& c_{\widetilde{E}_{8}}(N)=\left\{\begin{array}{c}
0 \text { if } n \text { or } n^{\prime}>0, \\
1 \frac{1}{4} \prod_{i=1}^{k}\left(n_{i}+1\right)\left(1-(-1)^{\sum_{i=1}^{k} n_{i}}\right) \prod_{i=1}^{l}\left(m_{i}+1\right) \\
\prod_{i=1}^{t}\left(1+(-1)^{s_{i}}\right) / 2^{t} \text { otherwise, }
\end{array}\right.
\end{aligned}
$$

for $N=2^{n} 3^{n^{\prime}} \prod_{i=1}^{k} p_{i}^{n_{i}} \prod_{i=1}^{l} q_{i}^{m_{i}} \prod_{i=1}^{t} r_{i}^{s_{i}}$ where $p_{i}$ is a prime number with $p_{i} \equiv$ 5 (12), $q_{i}$ is a prime number with $q_{i} \equiv 1$ (12) and $r_{i}$ is a prime number with $r_{i} \equiv 7$ or $11(12)$.

$$
\begin{aligned}
W=(1,1,2 ; 5) & \\
\eta_{(1,1,2 ; 5)}(\tau) & =\eta(5 \tau)^{5} / \eta(\tau) \\
& =q+q^{2}+2 q^{3}+3 q^{4}+5 q^{5}+2 q^{6}+6 q^{7}+5 q^{8}+7 q^{9}+5 q^{10}+12 q^{11}+\cdots \\
L_{(1,1,2 ; 5)}(s) & =\prod_{p} \frac{1}{\left(1-p^{-s+1}\right)\left(1-\left(\frac{p}{5}\right) p^{-s}\right)} \\
c_{(1,1,2 ; 5)}(N) & =\prod_{i=1}^{k}\left(\left(p_{i}^{n_{i}+1}-\left(\frac{p_{i}}{5}\right)^{n_{i}+1}\right) /\left(p_{i}-\left(\frac{p_{i}}{5}\right)\right)\right)
\end{aligned}
$$

for the prime decomposition $N=\prod_{i=1}^{k} p_{i}^{n_{i}}$. The Fourier expansion of the eta-product $\eta_{(1,1,2 ; 5)}$ was studied by Ramanujan, who called it a "remarkable identity" [R]. The weight system $(1,1,2 ; 5)$ belongs to the 4th group of Appendix 1. 


\section{Appendix 1. Weight systems of rank less than or equal to 24 .}

This appendix is added after discussions with K. Harada, T. Kondo and G. Mason. We compare the Frame shape (which is the same as the cyclotomic exponents in the present paper) for conjugacy classes of the automorphism group - 0 of the Leech lattice, with that of direct sums of weight systems having rank 24 defined below. We refer to $[\mathrm{C}-\mathrm{N}],[\mathrm{H}-\mathrm{L}],[\mathrm{K}]$ and $[\mathrm{Ma}]$ for the Frame shape of classes of $\cdot 0$ and related subject.

By a direct sum of weight systems, we mean a symbol $W_{1} \oplus \cdots \oplus W_{m}$, where $W_{i}$ are weight systems having the same Coxeter number. The rank (resp. characteristic polynomial and discriminant) of $W_{1} \oplus \cdots \oplus W_{m}$ is defined to be the sum (resp. the product) of these of the summands $W_{i}$.

In the following, we list up direct sums of weight systems having rank 24 (which is proceeded by a help of Table $\mathbf{F}$ at the end of Appendix 1). The list is divided into the four groups.

1) direct sum of weight systems having only positive exponents (cf. $\S 10$ ):

$$
\begin{aligned}
& 24 A_{1}, 12 A_{2}, 8 A_{3}, 6 A_{4}, 4 A_{5} \oplus D_{4}, 6 D_{4}, 4 A_{6}, 2 A_{7} \oplus 2 D_{5}, 3 A_{8}, \\
& 2 A_{9} \oplus D_{6}, 4 D_{6}, A_{11} \oplus D_{7} \oplus E_{6}, 4 E_{6}, 2 A_{12}, 3 D_{8}, A_{15} \oplus D_{9}, \\
& D_{10} \oplus 2 E_{7}, A_{17} \oplus E_{7}, 2 D_{12}, A_{24}, D_{16} \oplus E_{8}, 3 E_{8}, D_{24} .
\end{aligned}
$$

2) direct sum of dual pairs of simple weight system with $\varepsilon=-1$ (cf. $\S 11$ ):

$$
\begin{aligned}
& 2(3,4,4 ; 12), 2(3,4,5 ; 13),(4,5,6 ; 16) \oplus(3,4,8 ; 16), 2(3,5,6 ; 15), \\
& (4,6,7 ; 18) \oplus(3,5,9 ; 18),(6,8,9 ; 24) \oplus(3,8,12 ; 24), 2(4,5,10 ; 20), \\
& 2(4,6,11 ; 22),(6,8,15 ; 30) \oplus(4,10,15 ; 30), 2(6,14,21 ; 42) .
\end{aligned}
$$

3) weight system of rank 24 having negative exponents (cf: Table F):

$$
\begin{aligned}
& (3,7,7 ; 21),(3,7,11 ; 25),(3,11,15 ; 33),(4,7,12 ; 28),(4,9,18 ; 36), \\
& (4,14,23 ; 46),(6,9,11 ; 33),(6,10,23 ; 46),(6,26,39 ; 78), \\
& (10,14,35 ; 70),(12,15,20 ; 60) .
\end{aligned}
$$

4) degenerate weight systems:

$$
\begin{aligned}
& 3 \widetilde{E}_{6}, 2 \widetilde{E}_{6} \oplus 4 A_{2}, \widetilde{E}_{6} \oplus 8 A_{2}, 2 \widetilde{E}_{7} \oplus 2 A_{3}, \widetilde{E}_{7} \oplus 5 A_{3}, \\
& 2 \widetilde{E}_{8} \oplus D_{4}, \widetilde{E}_{8} \oplus 2 A_{5} \oplus D_{4},(1,2,2 ; 6) \oplus D_{4},(1,1,2 ; 5)
\end{aligned}
$$

We observe the following facts on the table, which are verified directly.

1. The characteristic polynomial of a direct sum in the lists 1), 2) and 3) is self-dual (in the sense at introduction),

2. The discriminant of a direct sum in the lists 1), 2) and 3) is a square.

3. The cyclotomic exponents of a direct sum in the list 1), 2) and 3) appears as a Frame shape of an element of the Conway group $\cdot 0$,

4. All of self-dual Frame shapes for conjugacy classes of 0 appear in this way, except for the 4 conjugacy classes: $6 A=6^{4} 3^{4} / 1^{4} 2^{4}, 10 A=10^{2} 5^{2} / 1^{2} 2^{2}, 15 D=30 \cdot 10 \cdot 6$. $2 / 1 \cdot 3 \cdot 5 \cdot 15$ and $18 A=18 \cdot 9 / 1 \cdot 2$.

5. All weight systems in the list 4 ) are degenerate ( $\Leftrightarrow$ the characteristic polynomial has 1 as its roots $\left.\Leftrightarrow a_{0}>0\right)$. Therefore, they are not self-dual and their cyclotomic 
exponents do not appear as a Frame shape of the Conway group $\cdot 0$. The Fourier coefficients of eta-products attached to them are non-negative integers (cf. Example 13.7).

REMARK A.1

1. The self-duality of direct sums in the lists 1) and 2) is a consequence of $\S 7$ Theorem 7.10.

2. The squareness of the discriminant for direct sums in the list 2) is a consequence of $\S 9$ Assertion 9.1 .

3. Let $L=L_{1} \oplus \cdots \oplus L_{m}$ be the direct sum of the lattices $L_{i}$ of vanishing cycles for $W_{i}$. It may be an interesting question whether there exists a unimodular lattice $\widetilde{L}$ such that $L \subset \widetilde{L} \subset L^{*}$ (= the dual lattice of $L$ ). In the case of 1 ), this is true because of the Niemeier lattice $\widetilde{L}$. In the case of 2 ), this is true because of the $K 3$-lattice $\widetilde{L}$. In the case of 3 ), the discriminants are squares, but we do not know the answer.

In Appendix 2, we introduce an algebraic surface $\widetilde{X}_{W}$ for each of the weight system $W$ in the group 3) so that the lattice of the vanishing cycles is realized as the lattice of transcendental cycles of the surface $\widetilde{X}_{W}$.

4. The weight system $(1,1,2 ; 5)$ in the list 4$)$ has a negative exponent -1 . But since it is degenerate, we classified it in the group 4) but not in 3). The eta-products for this case was studied by Ramanujan as remarkable identity [R] (see also Example 13.7).

In Table $\mathbf{F}$, we list up all weight systems of rank less or equal to 24 together with the data: rank $\mu_{W}$, discriminant $d(W)$, genus $a_{0}$, signature $A(W)$, characteristic polynomial $\varphi_{W}(\lambda)$ and the poset $M(W)$. We omit the case $\varepsilon \geq 0$ and the case $\varepsilon=-1$ of mult $(W)=1$, which are already listed in tables A, B, C and E. A weight system having a dual weight system is indicated either by "*" (in case self-dual) or by "**" (otherwise) and the dual weight is given in the table.

$$
\varepsilon=-1
$$

Table F.

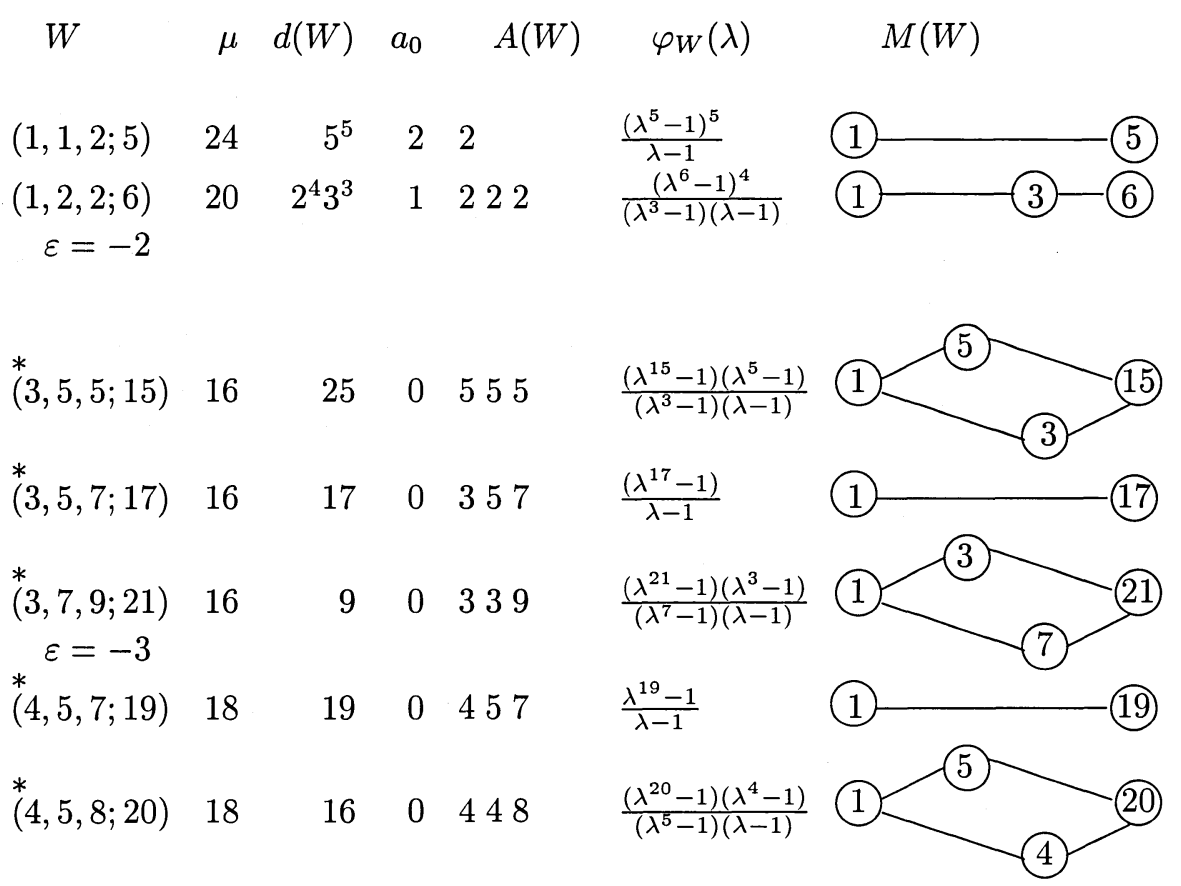


$\stackrel{*}{(4,7,14 ; 28)} \quad 18 \quad 7 \quad 0 \quad 277 \quad \frac{\left(\lambda^{28}-1\right)\left(\lambda^{7}-1\right)\left(\lambda^{2}-1\right)}{\left(\lambda^{14}-1\right)\left(\lambda^{4}-1\right)(\lambda-1)}$

$\stackrel{*}{(4,10,17 ; 34) \quad 18 \quad 4 \quad 0} \quad 2410 \quad \frac{\left(\lambda^{34}-1\right)\left(\lambda^{2}-1\right)}{\left(\lambda^{17}-1\right)(\lambda-1)}$

$$
\varepsilon=-4
$$

$\stackrel{*}{(3,7,7 ; 21)} 24 \quad 49 \quad 0 \quad 777 \quad \frac{\left(\lambda^{21}-1\right)\left(\lambda^{\top}-1\right)}{\left(\lambda^{3}-1\right)(\lambda-1)}$

$\stackrel{*}{(3,7,11 ; 25)} \quad 24 \quad 25 \quad 0 \quad 3711 \quad \frac{\lambda^{25}-1}{\lambda-1}$
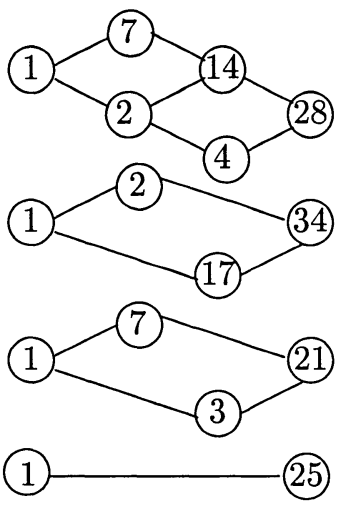

$\stackrel{*}{(3,11,15 ; 33)} 24 \quad 9 \quad 0 \quad 3315 \quad \frac{\left(\lambda^{33}-1\right)\left(\lambda^{3}-1\right)}{\left(\lambda^{11}-1\right)(\lambda-1)}$

$\varepsilon=-5$

$\stackrel{*}{(6,7,9 ; 27) \quad 20} \quad 9 \quad 0 \quad 367 \quad \frac{\left(\lambda^{27}-1\right)\left(\lambda^{3}-1\right)}{\left(\lambda^{9}-1\right)(\lambda-1)}$

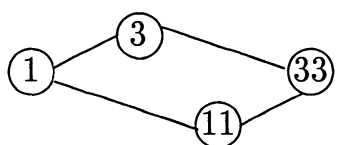

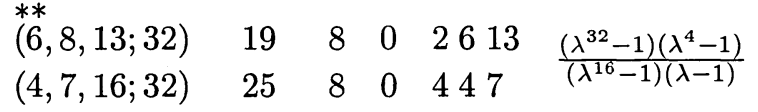

(1) (3) (27)

(1)-(4)-(32)

$\stackrel{*}{(6,8,19 ; 38)} 20 \quad 4 \quad 0 \quad 268 \quad \frac{\left(\lambda^{38}-1\right)\left(\lambda^{2}-1\right)}{\left(\lambda^{19}-1\right)(\lambda-1)}$

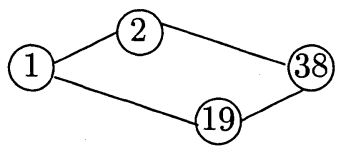

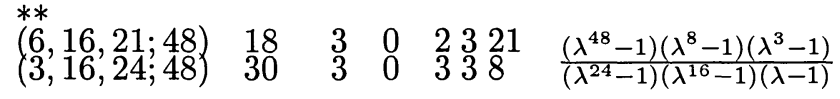<smiles>c1ccc2ccccc2c1</smiles>

$\begin{array}{lllllll}* * & (6,16,27 ; 54) & 19 & 2 & 0 & 2316 & \left(\lambda^{54}-1\right)\left(\lambda^{9}-1\right)\left(\lambda^{2}-1\right) \\ (4,18,27 ; 54) & 25 & 2 & 0 & 249 & \frac{\left(\lambda^{27}-1\right)\left(\lambda^{18}-1\right)(\lambda-1)}{(1)}\end{array}$

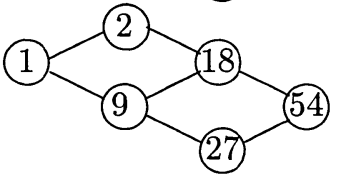

$\stackrel{*}{(6,22,33 ; 66)} 20 \quad 1 \quad 0 \quad 2311 \quad \frac{\left(\lambda^{66}-1\right)\left(\lambda^{11}-1\right)\left(\lambda^{3}-1\right)\left(\lambda^{2}-1\right)}{\left(\lambda^{33}-1\right)\left(\lambda^{22}-1\right)\left(\lambda^{6}-1\right)(\lambda-1)}$

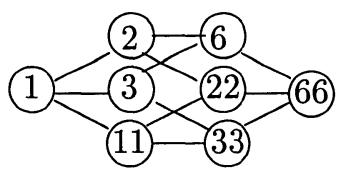

$\stackrel{*}{(4,7,12 ; 28)} \quad 24 \quad 16 \quad 0 \quad 4412 \quad \frac{\left(\lambda^{28}-1\right)\left(\lambda^{4}-1\right)}{\left(\lambda^{7}-1\right)(\lambda-1)}$

$(4,9,14 ; 32) \quad 23 \quad 16 \quad 0 \quad 2914 \quad \frac{\left(\lambda^{32}-1\right)\left(\lambda^{8}-1\right)}{\left(\lambda^{16}-1\right)(\lambda-1)}$

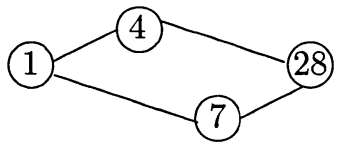

(1) (8)-(16)

$\stackrel{*}{(4,9,18 ; 36)} \quad 24 \quad 9 \quad 0 \quad 299 \quad \frac{\left(\lambda^{36}-1\right)\left(\lambda^{9}-1\right)\left(\lambda^{2}-1\right)}{\left(\lambda^{18}-1\right)\left(\lambda^{4}-1\right)(\lambda-1)}$<smiles></smiles>

$(4,14,19 ; 42) \quad 23 \quad 6 \quad 0 \quad 2419 \quad \frac{\left(\lambda^{42}-1\right)\left(\lambda^{3}-1\right)}{\left(\lambda^{21}-1\right)(\lambda-1)}$

(1) (3)-21 - (42) 
$\stackrel{*}{(4,14,23 ; 46)} 24 \quad 4 \quad 0 \quad 2414 \quad \frac{\left(\lambda^{46}-1\right)\left(\lambda^{2}-1\right)}{\left(\lambda^{23}-1\right)(\lambda-1)}$ $\varepsilon=-7$

$\stackrel{*}{(8,9,12 ; 36)} 21 \quad 4 \quad 0 \quad 348 \quad \frac{\left(\lambda^{36}-1\right)\left(\lambda^{4}-1\right)\left(\lambda^{3}-1\right)}{\left(\lambda^{12}-1\right)\left(\lambda^{9}-1\right)(\lambda-1)}$

$\begin{array}{lllllll}* * & (8,10,15 ; 40) & 20 & 5 & 0 & 2515 & \left(\lambda^{40}-1\right)\left(\lambda^{5}-1\right)\left(\lambda^{4}-1\right) \\ (5,8,20 ; 40) & 28 & 5 & 0 & 455 & \frac{1}{\left(\lambda^{20}-1\right)\left(\lambda^{8}-1\right)(\lambda-1)}\end{array}$

$\stackrel{*}{(8,10,25 ; 50)} 21 \quad 2 \quad 0 \quad 258 \quad \frac{\left(\lambda^{50}-1\right)\left(\lambda^{5}-1\right)\left(\lambda^{2}-1\right)}{\left(\lambda^{25}-1\right)\left(\lambda^{10}-1\right)(\lambda-1)}$

$(6,9,11 ; 33) \quad 24 \quad 9 \quad 0 \quad 369 \quad \frac{\left(\lambda^{33}-1\right)\left(\lambda^{3}-1\right)}{\left(\lambda^{11}-1\right)(\lambda-1)}$

$(6,10,13 ; 36) \quad 23 \quad 12 \quad 0 \quad 21013 \quad \frac{\left(\lambda^{36}-1\right)\left(\lambda^{6}-1\right)}{\left(\lambda^{18}-1\right)(\lambda-1)}$

$(6,10,23 ; 46) \quad 24 \quad 4 \quad 0 \quad 2610 \quad \frac{\left(\lambda^{46}-1\right)\left(\lambda^{2}-1\right)}{\left(\lambda^{23}\right)(\lambda-1)}$

$(6,20,27 ; 60) \quad 22 \quad 3 \quad 0 \quad 2327 \quad \frac{\left(\lambda^{60}-1\right)\left(\lambda^{10}-1\right)\left(\lambda^{3}-1\right)}{\left(\lambda^{30}-1\right)\left(\lambda^{20}-1\right)(\lambda-1)}$

$(6,20,33 ; 66) \quad 23 \quad 2 \quad 0 \quad 2320 \quad \frac{\left(\lambda^{66}-1\right)\left(\lambda^{11}-1\right)\left(\lambda^{2}-1\right)}{\left(\lambda^{33}-1\right)\left(\lambda^{22}-1\right)(\lambda-1)}$

$(6,26,39 ; 78) \quad 24 \quad 1 \quad 0 \quad 2313 \quad \frac{\left(\lambda^{78}-1\right)\left(\lambda^{13}-1\right)\left(\lambda^{3}-1\right)\left(\lambda^{2}-1\right)}{\left(\lambda^{39}-1\right)\left(\lambda^{26}-1\right)\left(\lambda^{6}-1\right)(\lambda-1)}$

$$
\varepsilon=-11
$$

$(10,14,35 ; 70) \quad 24 \quad 1 \quad 0 \quad 257 \quad \frac{\left(\lambda^{70}-1\right)\left(\lambda^{7}-1\right)\left(\lambda^{5}-1\right)\left(\lambda^{2}-1\right)}{\left(\lambda^{35}-1\right)\left(\lambda^{14}-1\right)\left(\lambda^{10}-1\right)(\lambda-1)}$

$$
\varepsilon=-13
$$

$\stackrel{*}{*}, 15,20 ; 60) \quad 24 \quad 1 \quad 0 \quad 345 \quad \frac{\left(\lambda^{60}-1\right)\left(\lambda^{5}-1\right)\left(\lambda^{4}-1\right)\left(\lambda^{3}-1\right)}{\left(\lambda^{20}-1\right)\left(\lambda^{15}-1\right)\left(\lambda^{12}-1\right)(\lambda-1)}$

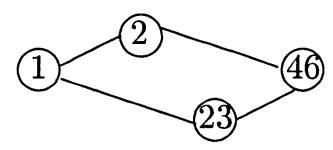

(1) (3) (3)

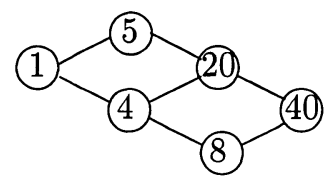

(1)

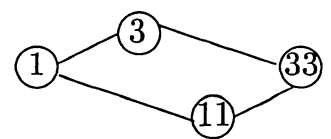

(1)-(6)-(18)

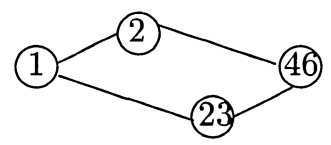

(1)
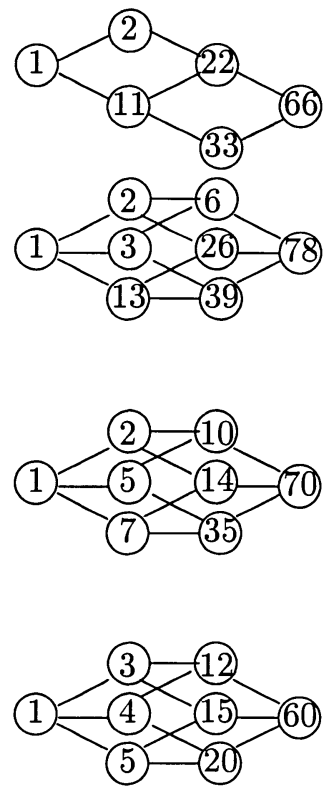


\section{Appendix 2. Algebraic Surfaces for Weight Systems of Rank 24.}

To each $W$ of the 11 weight systems of rank 24 in the list 3) of Appendix 1, we attach an algebraic surface $\widetilde{X}_{W}$ and its lattice $L_{W}$ of transcendental cycles of rank 24. In all 11 cases, the number of negative exponents is equal to 2 , and hence the signature of the quadratic form of the lattice is $(4,20)$. It is remarkable that, in all 11 cases, the discriminant of the lattice is a square integer. It would be interesting to find a characterization of these lattices.

First, we recall the list of the weight systems together with the data: i) characteristic polynomial $\varphi=\varphi_{W}$, ii) discriminant $d=d(W)$, iii) signature set $A=A(W)$, iv) volume vol, and v) exponents. Of course $\mu_{W}=\nu_{W}=24$ and $a_{0}=0$.

$$
(3,7,7 ; 21)
$$

$$
\begin{aligned}
& \varphi=21 \cdot 7 / 3 \cdot 1, d=49, A=\{7,7,7\}, \mathrm{vol}=4 / 7, \\
& \text { exponents }:-4,-1,2,3,3,5,6,6,8,9,9,10,11,12,12,13,15,15,16,18,
\end{aligned}
$$

$18,19,22,25$

$$
(3,7,11 ; 25)
$$

$$
\varphi=25 / 1, d=25, A=\{3,7,11\}, \text { vol }=4 / 7 \text {, }
$$

exponents : $-4,-1,2,3,5,6,7,8,9,10,11,12,13,14,15,16,17,18,19,20$,

$22,23,26,29$

$$
(3,11,15 ; 33)
$$

$\varphi=33 \cdot 3 / 11 \cdot 1, d=9, A=\{3,3,15\}$, vol $=4 / 15$,

exponents : $-4,-1,2,5,7,8,10,11,11,13,14,16,17,19,20,22,22,23,25$,

$26,28,31,34,37$

$(4,7,12 ; 28)$

$\varphi=28 \cdot 4 / 7 \cdot 1, d=16, A=\{4,4,12\}$, vol $=5 / 12$,

exponents : $-5,-1,2,3,6,7,7,9,10,11,13,14,14,15,17,18,19,21,21$,

$22,25,26,29,33$

$$
(4,9,18 ; 36)
$$

$\varphi=36 \cdot 9 \cdot 2 / 18 \cdot 4 \cdot 1, d=9, A=\{2,9,9\}$, vol $=7 / 18$,

exponents : $-5,-1,3,4,7,8,11,12,13,15,16,17,19,20,21,23,24,25,28,29$,

$32,33,37,41$

$$
(4,14,23 ; 46)
$$

$\varphi=46 \cdot 2 / 23 \cdot 1, d=4, A=\{2,4,14\}, \mathrm{vol}=5 / 23$,

exponents : $-5,-1,3,7,9,11,13,15,17,19,21,23,25,27,29,31,33,35,37,39$,

$$
43,47,51
$$

$(6,9,11 ; 33)$

$\varphi=33 \cdot 3 / 11 \cdot 1, d=9, A=\{3,6,9\}$, vol $=7 / 18$,

exponents : $-7,-1,2,4,5,8,10,11,11,13,14,16,17,19,20,22,22,23,25,28,29$,

$31,34,40$

$(6,10,23 ; 46)$

$\varphi=46 \cdot 2 / 23 \cdot 1, d=4, A=\{2,6,10\}$, vol $=7 / 30$,

exponents : $-7,-1,3,5,9,11,13,15,17,19,21,23,23,25,27,29,31,33,35,37$, 


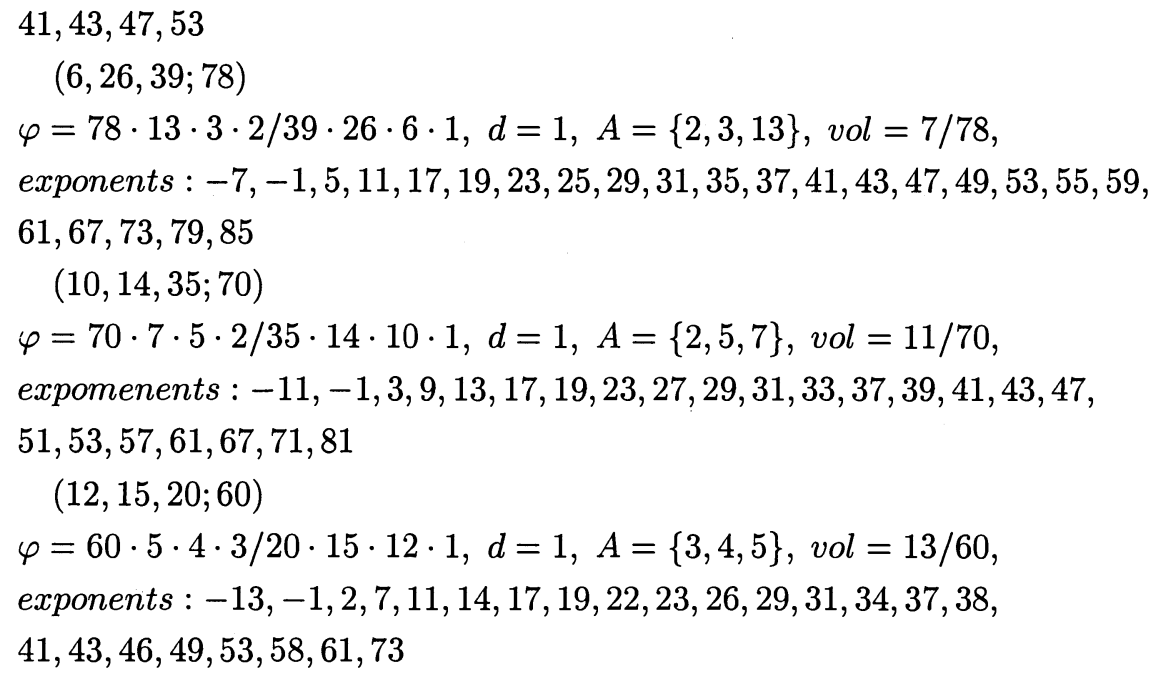

According to $\S 10$ Remark 10.3 , any weight system $W=(a, b, c ; h)$ defines a smooth curve $C_{W}$ in $\mathbb{P}(a, b, c)$ by a weighted homogeneous polynomial $f_{W}$ of three variables $(x, y, z)$ of weight $a, b$ and $c$ and of total degree $h$. The genus $g\left(C_{W}\right)$ (which is shown to be equal to $a_{0}$ ) turns out to be zero for all of the 11 weight systems. The curve $C_{W}$ is given as an orbifold $\Gamma_{W} \backslash \mathcal{H}$, where $\Gamma_{W} \subset P S L_{2}(\mathbb{P})$ is a triangle group with the signature set $A(W)$. That is: there are 3 conjugacy classes of elliptic fixed points of $\Gamma_{W}$, where the set of orders of the fixed points is $A(W)$. Since the polynomial $f_{W}$ consists of 3 monomials ( $\left(10\right.$ Remark 3 ), $f_{W}$ is unique up to constant factors on coordinates. The affine equation $f_{W}=0$ in $\mathbb{C}^{3}$ defines a surface $X_{W, 0}$ with an isolated singular point at the origin. The map $f_{W}: \mathbb{C}^{3} \backslash X_{W, 0} \rightarrow \mathbb{C} \backslash\{0\}$ defines a Milnor fibration whose general fiber $X_{W}$ (given by the affine equation: $f_{W}=1$ ) has a homotopy type of bouquet of $\mu_{W}$ number of 2-spheres [M]. We consider the middle (2-dim.) homology group $L_{W}:=-H_{2}\left(X_{W}, \mathbb{Z}\right)$. The $\operatorname{rank}\left(L_{W}\right)$ is given by $\mu_{W}$, and the sign $\left(\mu_{+}, \mu_{0}, \mu_{-}\right)$of $L_{W}$ is given by $\mu_{+}=\#\{$ exponents between 0 and $h\}, \mu_{0}=$ $\#\{$ exponents equal to 0 or $h\}, \mu_{-}=\#\{$ exponents which exceeds the above range $\}$. In fact, $\mu_{+}=16+4, \mu_{0}=0$ and $\mu_{-}=4$ in all of the 11 weight systems. The discriminant of $L_{W}$ is given by $d(W)$.

In order to give a geometric description of the lattice $L_{W}$, we describe Pinkham's compactification of the surface $X_{W}$ and its smooth model (cf. [P1 3]). Let $\bar{X}_{W}$ be the surface in the weighted projective space $\mathbb{P}(a, b, c, 1)=\mathbb{C}^{3} \cup \mathbb{P}(a, b, c)$ defined by the equation $f_{W}(x, y, z)+w^{h}$. So, $\bar{X}_{W}=X_{W} \cup C_{W}$, where $C_{W}=\bar{X}_{W} \cap \mathbb{P}(a, b, c)$. The singularities of $\bar{X}_{W}$ are cyclic quotient singularities exactly at the orbifold points of $C_{W}$ by a group of order given by the signature $A(W)$. By blowing up them, we obtain a smooth model $\widetilde{X}_{W}$ of $\bar{X}_{W}$, which turns out to be minimal, checked case by case for 11 cases. Let $D_{\infty}=\widetilde{X}_{W} \backslash X_{W}$ be the total transform of the curve $C_{W}$ in $\widetilde{X}_{W}$. Let $\mathbb{Z}\left[D_{\infty}\right]$ be the submodule of $H_{2}\left(\widetilde{X}_{W}, \mathbb{Z}\right)$ spanned by irreducible components of $D_{\infty}$. Then we have the description:

$$
L_{W} \cong \mathbb{Z}\left[D_{\infty}\right]^{\perp}
$$

(cf. [S7 (5.9.1)]). The determinant of the intersection matrix of the irreducible components of $D_{\infty}$ is equal to the discriminant $d(W)$ (checked case by case). So the irreducible components are linearly independent in $H_{2}\left(\widetilde{X}_{W}, \mathbb{Z}\right)$. On the other hand, 
the Picard number for the del Sart surface $\tilde{X}_{W}$ turns out to be equal to $\operatorname{rank}\left(\mathbb{Z}\left[D_{\infty}\right]\right)$ owing to an explicit formula [Shi]. These altogether implies the next statement:

AsSERTION A.2 The lattice $L_{W}$ is isomorphic to the lattice of the transcendental cycles of the surface $\widetilde{X}_{W}$ whose discriminant is equal to $d(W)$.

Furthermore, in 9 of the 11 cases, $\widetilde{X}_{W}$ has Kodaira dimension 1 and admits an elliptic fibration over a rational curve, and in the remaining two cases, $\widetilde{X}_{W}$ has Kodaira dimension 2 and admits genus 2 or 3 fibrations over a rational curve.

In Table $\mathbf{H}$, for each of 11 weight systems $W$, we exhibit the data:

i) Weighted homogeneous polynomial: $f_{W}+w^{h}$ defining $\bar{X}_{W}$.

ii) The Chern numbers $c_{1}^{2}=K_{1}^{2}$ and $c_{2}$, geometric genus $p_{g}$, and the Kodaira dimension $\kappa$ of the surface $\widetilde{X}_{W}$.

iii) Intersection diagram of the exceptional set $E_{0}$ of the minimal resolution of $X_{W, 0}$ is given by the figure in the left side. Irreducible components of $E_{0}$ are rational curves whose self-intersection numbers are given by the numbers inside the circle. The coefficients of the canonical divisor of the resolution are given near to the circle.

iv) The intersection diagram of $D_{\infty}$ is given by the figure in the right hand side. Irreducible components of $D_{\infty}$ are relational curves. The coefficients of the canonical divisor of the surface $\widetilde{X}_{W}$ are given near at the vertices.

Table H.

$$
\tilde{X}_{w}:=\left\{x^{7}+y^{3}+z^{3}+w^{21}=0\right\}
$$

$K_{\infty}\left(\tilde{X}_{w}\right)^{2}=0, \quad c_{2}\left(\tilde{X}_{w}\right)=36, \quad p_{g}\left(\tilde{X}_{w}\right)=2, \quad \kappa\left(\tilde{X}_{w}\right)=1$.
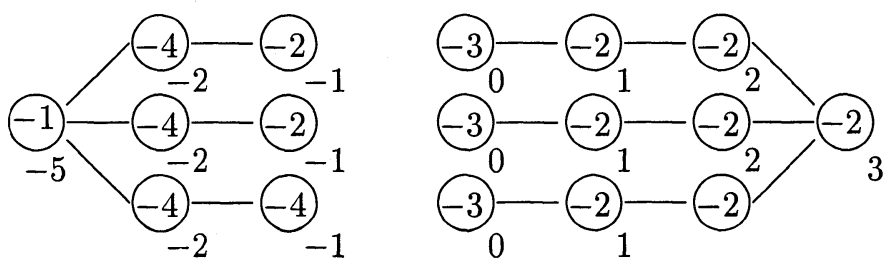

$(3,7,11 ; 25)$

$$
\widetilde{X}_{w}:=\left\{x^{6}+y+y^{2} z+z^{2}+w^{25}=0\right\}
$$

$$
K_{\infty}\left(\widetilde{X}_{w}\right)^{2}=0, \quad c_{2}\left(\widetilde{X}_{w}\right)=36, \quad p_{g}\left(\tilde{X}_{w}\right)=2, \quad \kappa\left(\widetilde{X}_{w}\right)=1
$$
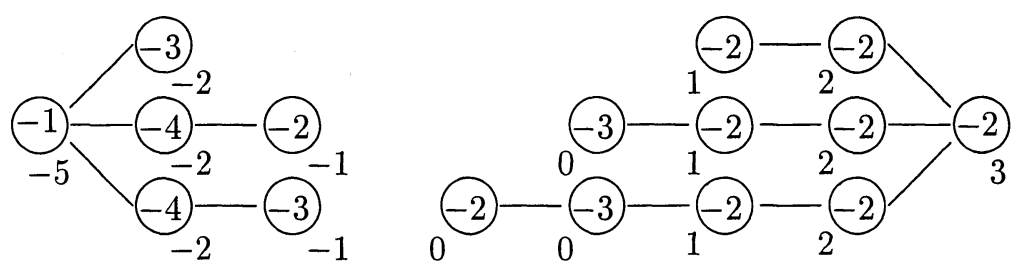

$(4,7,12 ; 28)$

$$
\tilde{X}_{w}:=\left\{x^{7}+y^{4}+z^{2} x+w^{28}=0\right\}
$$


$K_{\infty}\left(\tilde{X}_{w}\right)^{2}=0, \quad c_{2}\left(\widetilde{X}_{w}\right)=36, \quad p_{g}\left(\tilde{X}_{w}\right)=2, \quad \kappa\left(\tilde{X}_{w}\right)=1$
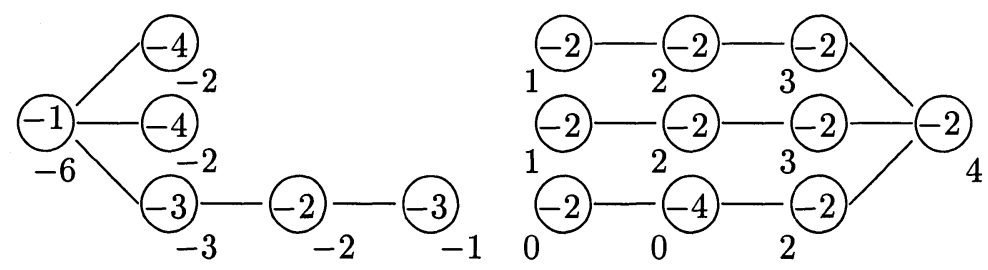

$(4,14,23 ; 46) \quad \tilde{X}_{w}:=\left\{x^{8} y+y^{3} x+z^{2}+w^{46}=0\right\}$

$K_{\infty}\left(\widetilde{X}_{w}\right)^{2}=0, \quad c_{2}\left(\widetilde{X}_{w}\right)=36, \quad p_{g}\left(\widetilde{X}_{w}\right)=2, \quad \kappa\left(\widetilde{X}_{w}\right)=1$.

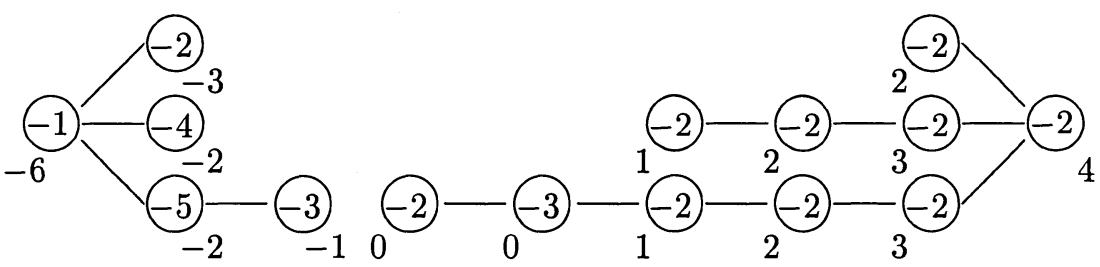

$(6,9,11 ; 33) \quad \widetilde{X}_{w}:=\left\{x^{4} y+y^{3} x+z^{3}+w^{33}=0\right\}$

$K_{\infty}\left(\tilde{X}_{w}\right)^{2}=0, \quad c_{2}\left(\tilde{X}_{w}\right)=36, \quad p_{g}\left(\tilde{X}_{w}\right)=2, \quad \kappa\left(\tilde{X}_{w}\right)=1$.

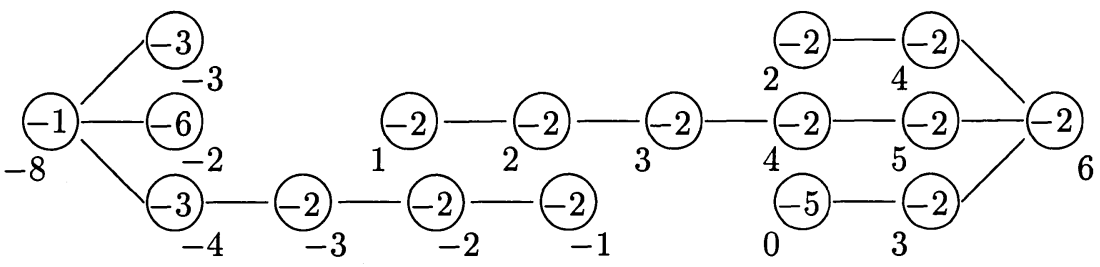

$(6,10,23 ; 46) \quad \widetilde{X}_{w}:=\left\{x^{6} y+y^{4} x+z^{2}+w^{46}=0\right\}$

$K_{\infty}\left(\tilde{X}_{w}\right)^{2}=0, \quad c_{2}\left(\widetilde{X}_{w}\right)=36, \quad p_{g}\left(\tilde{X}_{w}\right)=2, \quad \kappa\left(\tilde{X}_{w}\right)=1$

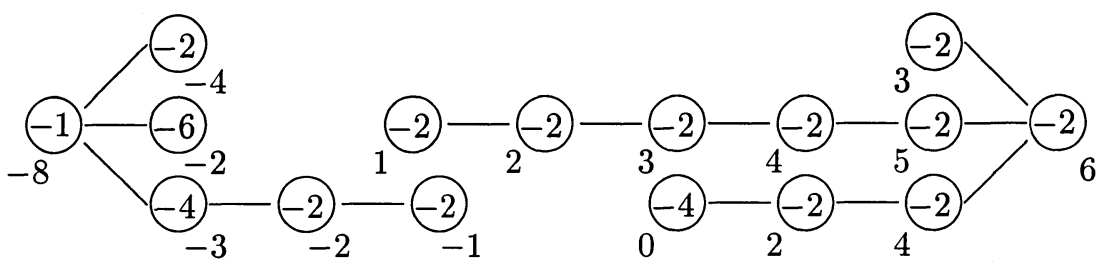

$(6,26,39 ; 78) \quad \tilde{X}_{w}:=\left\{x^{13}+y^{3}+z^{2}+w^{78}=0\right\}$ 


$$
K_{\infty}\left(\tilde{X}_{w}\right)^{2}=0, \quad c_{2}\left(\tilde{X}_{w}\right)=36, \quad p_{g}\left(\tilde{X}_{w}\right)=2, \quad \kappa\left(\widetilde{X}_{w}\right)=1
$$

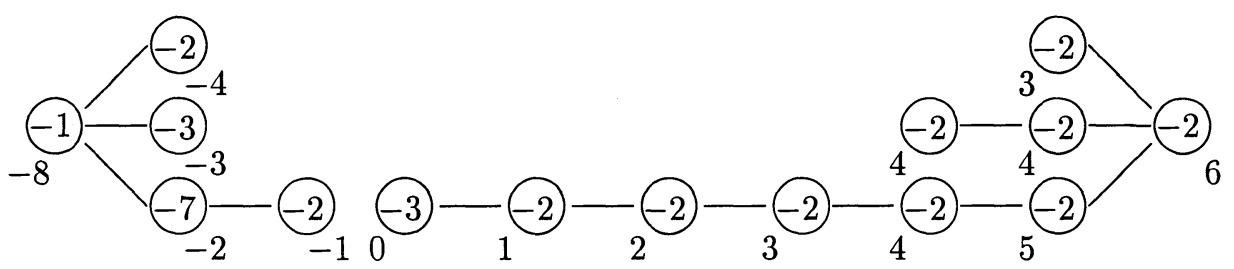

$$
\begin{aligned}
&(10,14,35 ; 70) \quad \widetilde{X}_{w}:=\left\{x^{7}+y^{5}+z^{2}+w^{70}=0\right\} \\
& K_{\infty}\left(\widetilde{X}_{w}\right)^{2}=1, \quad c_{2}\left(\widetilde{X}_{w}\right)=35, \quad p_{g}\left(\widetilde{X}_{w}\right)=2, \quad \kappa\left(\widetilde{X}_{w}\right)=2 .
\end{aligned}
$$
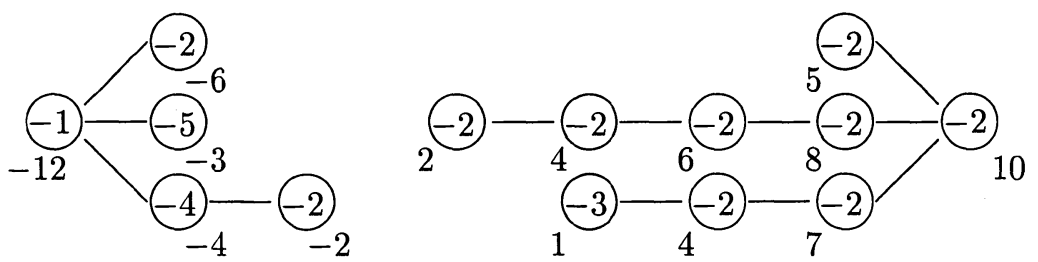

$(12,15,20 ; 60) \quad \tilde{X}_{w}:=\left\{x^{5}+y^{4}+z^{3}+w^{60}=0\right\}$

$$
K_{\infty}\left(\tilde{X}_{w}\right)^{2}=2, \quad c_{2}\left(\tilde{X}_{w}\right)=34, \quad p_{g}\left(\tilde{X}_{w}\right)=2, \quad \kappa\left(\tilde{X}_{w}\right)=2
$$
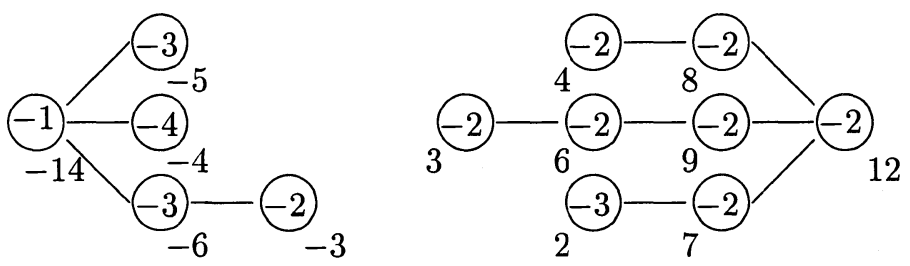

\section{REFERENCES}

[A1] V. I. ARnold, Critical Points of Smooth Functions, Proc. Internat. Congress Math., Vancouver, I, 1974, pp. 19.

[A2] - Normal forms of function in neighborhood of degenerate critical points, Usp. Mat. Nauk, 29:2 (1974), pp. 11-49.

[A3] - Critical points of smooth functions and their normal forms, Usp. Mat. Nauk., 30:5 (1975), pp. 3-65.

[Bo] N. Bourbaki, Groups Et Algèbres De Lie, Hermann, Paris, 1968.

[B1] E. BRIESKORN, Die Habilitationsschrift, Bonn, 1965.

[B2] - Beispiele zur differential topology von Singularitäten, Invent. Math., 2 (1966), pp. 1-14.

[B3] — Die Milnorgitter der Exzeptionellen und Unimodularen Singularitäten, Bonner Math. Schriften, No. 150, Bonn, 1983.

[C-N] J. H. Conway And S. P. Norton, Monstrous Moonshine, Bull. London Math., 11 (1979), pp. 308-339. 
[D1] I. V. Dolgachev, Quotient-Conical singularities on complex surfaces, Funk. Anal. i Prilozen, 8 (1974), pp. 160-161.

[D2] - Automorphic forms and quasihomogeneous singularities, Funk. Anal. i Prilozen, 9:2 (1975), pp. 67-68.

[D3] - On the link space of a Gorenstein quasihomogeneous surface singularities, Math. Ann., 232 (1978), pp. 85-08.

[E1] W. EBELING, Quadratische Formen und Monodrmie gruppen von Singularitäten, Math. Ann., 255 (1981), pp. 463-498.

[E2] - Milnor lattices and geometric bases of some special singularities, L'Einseignement Math., 29 (1983), pp. 263-280.

[E3] - An arithmetic characterization of the symmetric monodromy groups of singularities, Invent. Math., 77 (1984), pp. 85-99.

[E4] - Strange duality, mirror symmetry, and the Leech lattice, preprint 1996, alggeom $/ 9612010$.

[E-W] W. Ebeling AND T. WALL, Kodaira Singularities and an Extension of Arnold's Strange Duality, Compositio Math., 56:1 (1985), pp. 3-77.

[G] A. M. Gabrielov, Dynkin diagrams of unimodal singularities, Funck. Anal. i Prilozh., 8:3 (1974), pp. 1-6,

[H] Marshall JR. Hall, Combinatorial Theory, Blaisdell Publishing Company, 1967.

[H-L] K. Harada And L. L. Mong, Some Elliptic curves arising from the Leech lattice, J. Alg., 125 (1989), pp. 298-310.

[H-M] T. HONDA AND I. MIYAWAKI, Zeta functions of elliptic curves of 2-power conductor, J. Math. Soc. Japan, 26 (1974), pp. 362-373.

[Kob] M. KoBAYASHI, Duality of weights, mirror symmetry and Arnold's strange duality, preprint, 1994.

[K] T. KonDo, The automorphism group of Leech lattice and elliptic modular functions, J. Math. Soc. Japan, 37 (1985), pp. 337-362.

[Ko] M. KoIKE, Moonshines of $\mathrm{PSL}_{2}\left(F_{q}\right)$ and the automorphism group of Leech lattice, Japan. J. Math., 12:2 (1986), pp. 283-323.

[Le] TrÁNG LÊ DŨNG, Calcul des cycles evanouissants des hypersurfaces complexes, Ann. Inst. Fourier, 23 (1973), pp. 261-270.

[Lo] E. LoolJenga, Rational surfaces with an anti-canonical cycle, Ann. of Math., 114 (1981), pp. 267-322.

[Mag] W. Magnus, Noneuclidean Tesselations and Their Groups, Academic Press, New York and London, 1974.

[Man] Y. I. MANIN, Three constructions of Frobenius manifolds: a comparative study, to appear in Asian J. Math.

[Ma] G. MASON, Frame shapes and rational characters of finite groups, J. Alg., 89 (1984), pp. 237-246.

[Ma] - $M_{24}$ and certain automorphic forms, Comtemp. Math., 45 (1985), pp. 223-244.

[M] J. Milnor, Singular Points of Complex Hypersurfaces, Ann. of Math. Stud., 61, Princeton Univ. Press, 1968.

[M-O] J. MILNOR AND P. ORLIK, Isolated singularities defined by weighted homogeneous polynomials, Topology, 9 (1970), pp. 385-393.

[N] I. NAKAMURA, Inoue-Hirzebruch surfaces and a duality of hyperbolic unimodular singularities I, Math. Ann., 252 (1980), pp. 221-235.

[Oc] H. OchIAI, A personal letter to I. Satake (1992 Feb.).

[Od] T. ODA, Introduction to Algebraic Singularities, (with an Appendix by Taichi Ambai) Preprint, Lecture at Tata. Inst.

[O-W] P. ORLIK AND P. WAGREICH, Isolated singularities with $\mathbb{C}^{*}$-actions, Ann. Math., 93 (1971), pp. 205-228.

[O] M. OKA, Principal zeta-function of non-degenerate complete intersection singularity, J. Fac. Sci., Univ. Tokyo, Sect. IA Math., 31:1 (1990), pp. 11-32.

[P] H. PETERSON, Über die arithmetischen Eigenschaften eines Systems multiplikativer Modulefunkionen von Primzahlstufe, Acta Math., 95 (1956), pp. 57-110.

[P1] H. Pinkham, Singularitès exceptionelles, la dualite etrange d'Arnol'd et les surfaces $K$-3, C. R. Acad. Sci. Paris, 284 (A) (1977), pp. 615-618.

[P2] - Normal surface singularities with $C^{*}$-action, Math. Ann., 227 (1977), pp. 183-193.

[P3] - Deformation of Normal Surface Singularities with $\mathbb{C}^{*}$-action, Math. Ann, 232 (1978), pp. 65-84.

[Ra] H. RADEMACHER, Topics in Analytic Number Theory, Springer-Verlag, Berlin, Heidelberg, New York, 1973. 
[R] Eds. G. E. Andrews, R. A. Askey, B. C. Berndt, K. G. Ramanathan, and R. A. RANkin, Ramanujan Revisited, Proceedings of the Centenary Conference, Univ. of Illinois at Urbana-Champaign, June 1-5, 1987, Academic Press.

[S1] K. SAITO, Quasihomogene isolierte Singularitäten von Hyperflächen, Invent. Math., 14 (1971), pp. 123-142.

[S2] — Einfach-elliptische Singularitäten, Invent. Math. 23 (1974), pp. 289-325.

[S3] — Period Mapping Associated to a Primitive form, Publ. RIMS, Kyoto Univ., 19 (1983), pp. 1231-1264.

[S41] — Extended Affine Root Systems, I (Coxeter transformations), Publ. RIMS, Kyoto Univ., 21 (1985), pp. 75-179.

[S42] — - Extended Affine Root Systems, II (Flat Invariants), Publ. RIMS, Kyoto Univ., 26 (1990), pp. 15-78.

[S43] K. SaIto and T. TAKebayashi, Extended Affine Root Systems, III (Elliptic Weyl groups), Publ. RIMS, Kyoto Univ., 33 (1997), pp. 301-329.

[S44] K. SaITo AND D. Yoshil, Extended Affine Root Systems, IV (Elliptic Lie algebras), in preparation.

[S45] K. SAITo, Extended Affine Root Systems, V (Elliptic Eta-product and L-function), preprint RIMS-1210 June 1998.

[S5] — A New Relation among Cartan Matrix and Coxeter Matrix, J. Alg., 105 (1987), pp. 149-158.

[S6] - Regular system of weights and their associated singularities, Complex Analytic Singularities, Adv. Stud. Pure Math., 8 (1987), pp. 472-596.

[S7] - Algebraic surfaces associated to regular systems of weights, Algebraic Geometry and Commutative Algebra in Honor of Masayoshi Nagata, 2 (1988), pp. 517-614.

[S8] - On the existence of exponents prime to the Coxeter number, J. Alg., 114 (1988), pp. 333-356.

[S9] - - On a Linear Structure of a Quotient Variety by a Finite Reflexion Group, Publ. RIMS, Kyoto Univ., 29 (1993), pp. 535-579.

[S10] - On the Characteristic Polynomial for a Regular System of Weights, Preprint RIMS875, 1992.

On a Duality of Characteristic Polynomials for Regular Systems of Weights, Preprint RIMS-993, 1994.

[S11] - Around the theory of the generalized weight system: relations with singularity theory, the generalized Weyl group and its invariant theory, etc., Amer. Math. Soc. Transl. 2 (1997), pp. 101-143.

[S12] — Duality for Regular Systems of Weights, a precis, Proceedings of Taniguchi Symposium on Topological Field Theory, Primitive Forms and Related subjects, Birkauser, 1997.

[Sh] T. SHIODA, An explicite algorithm for computing the Picard number of certain algebraic surfaces, Amer. J. of Math., 108 (1986), pp. 415-432.

[T] A. TAKAHASHI, K. Saito's Duality for Regular Weight Systems and Duality for Orbifoldized Poincare Polynomials, math/9801050, to appear in Comm. Math. Phys..

[Wa1] P. WAGREICH, The structure of quasihomogeneous singularities, Proc. Symp. Pure Math. AMS, 40 (1973), pp. 593-624.

[Wa2] — Algebra of automorphic form with few generators, Trans. AMS, 262 (1980), pp. 367-389.

[Wah] J. WAHL, A characterization of Quasi-homogeneous Gorenstein surface singularities, Compositio Math., 55 (1985), pp. 269-288.

[Va] A. N. VARChEnKo, Zeta-function of Monodromy and Newton's Diagram, Invent. Math., 37 (1976), pp. 253-262. 
